Scanning tunneling microscopy studies of surface structures of icosahedral Al-Cu-Fe quasicrystals

by

\title{
Tanhong Cai
}

A dissertation submitted to the graduate faculty

in partial fulfiliment of the requirements for the degree of

DOCTOR OF PHILOSOPHY

Major: Physical Chemistry

Program of Study Committee:

Patricia A. Thiel, Major Professor

Klaus Schmidt-Rohr

Mei Hong

Valerie V. Sheares

Alan I. Goldman

Iowa State University

Ames, Iowa

2001

Copyright $(C$ Tanhong Cai, 2001. All rights reserved. 
Graduate College

Iowa State University

This is to certify that the doctoral dissertation of

Tanhong Cai

has met the dissertation requirements of Iowa State University

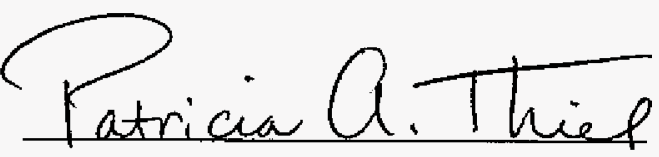

Major Professor

For the Major Program 
iii

Dedicated to my family 


\section{TABLE OF CONTENTS}

GENERAL INTRODUCTION

Dissertation Organization

References

STRUCTURAL ASPECTS OF THE FIVEFOLD QUASICRYSTALLINE AL-CU-FE SURFACE FROM STM AND DYNAMICAL LEED STUDIES

Abstract

12

1. Introduction 13

2. Experimental Description 16

3. Experimental STM Data 18

4. Sketch of Dynamical LEED Theory for Quasicrystalline Surfaces 22

5. LEED Structural Analysis 27

6. Conclusions 33

Acknowledgements $\quad 35$

References 35

AN STM STUDY OF ATOMIC STRUCTURE OF THE ICOSAHEDRAL AL-CU-FE FIVEFOLD SURFACE

Abstract $\quad 57$

Acknowledgements $\quad 63$

$\begin{array}{ll}\text { References } & 64\end{array}$

PSEUDOMORPHIC STARFISH: ARRANGEMENT OF EXTRINSIC METAL ATOMS ON A QUASICRYSTALLINE SUBSTRATE

$\begin{array}{ll}\text { Acknowledgements } & 79\end{array}$

$\begin{array}{ll}\text { References } & 79\end{array}$

$\begin{array}{ll}\text { GENERAL CONCLUSIONS } & 85\end{array}$ 
APPENDIX A. STRUCTURAL ASPECTS OF THE THREEFOLD SURFACE OF ICOSAHEDRAL AL-PD-MN

Abstract

Acknowledgements

References

APPENDIX B. ALUMINUM EVAPORATOR

APPENDIX C. STRUCTURE OF ALUMINUM FILM DEPOSITED ON ICOSAHEDRAL AL-CU-FE FIVEFOLD SURFACE

Abstract

106

1. Introduction

106

2. Experimental Description

3. Experimental Results

4. Discussions

4.1 Identification of the nucleation sties

4.3 Analysis on other atomic planes

4.4 The growth of the film with increasing coverage

5. Conclusions

Acknowledgements

References 


\section{GENERAL INTRODUCTION}

In classical crystallography, a crystal is defined as a space lattice plus a basis of atoms associated with each lattice point [1]. It can be generated from periodic translations of a unit cell and thus possesses a long-range translational order and an orientational order. Since the translational invariance forbids rotational symmetry of order $2 \pi / n$ for $n \neq 1,2,3,4$ or 6 , a crystal cannot have a fivefold or higher than sixfold rotational axis.

Quasicrystals, first discovered by Shechtman et al. [2] from the rapidly quenched AlMn alloy, are a new form of condensed matter in terms of order and symmetry. Quasicrystals have unique atomic structure in the sense that they are non-periodic but well ordered (namely quasiperiodic order), and typically they exhibit rotational symmetries that are forbidden in conventional crystallography, such as fivefold axes [3]. Since their first discovery, numerous quasicrystalline phases have been found in hundreds of compounds, which finally led the International Union of Crystallography to establish a new definition for "crystal" as "any solid with an essentially discrete diffraction pattern" in 1992 [4].

Besides their unique structures, quasicrystals are often found to have unusual physical properties. Although quasicrystals are typically binary or ternary alloys consisting of normal metallic elements, they are often poor electrical and poor thermal conductors. Their electrical resistivity is remarkably higher than their constituents and the resistivity increases with decreasing temperature [5]. All known quasicrystals are hard and brittle [6]. Some of their unusual physical properties involve surface phenomena, such as low coefficient of static friction, good corrosion resistance and non-stick behaviour $[7,8]$. These properties have attracted interest in using quasicrystals for many technology applications. 
Cookware with hard, non-stick and corrosion resistant quasicrystalline coatings have been commercially produced. Various potential applications, for instance, using quasicrystals as thermal barrier coatings, thermo-electric elements for small-scale heating and cooling, hydrogen storage materials, are being explored in research laboratories [9].

A common family of quasicrystals is the icosahedral type, which is quasiperiodic in three dimensions (3D). The discovery of thermodynamically stable icosahedral (i) quasicrystals in A1-Cu-Fe and AI-Pd-Mn systems by Tsai and his co-workers [10,11] promoted a significant development in the studies of both structure and physical properties of quasicrystals. The lack of periodicity makes structure determination for quasicrystals never an easy task, and it still remains an open question in spite of tremendous progress in recent years. Most of the work has been carried out on icosahedral (i-AlCuFe, i-AlPdMn) and decagonal systems, for which high quality single grain samples are available.

Several bulk models for atomic structures of icosahedral quasicrystals (both iAlPdMn and $\mathrm{i}-\mathrm{AlCuFe}$ ) have been derived and are still being modified. From 6D Patterson analysis of X-ray and neutron diffraction of i-AIPdMn single grain, Boudard and Janot $[12,13,14]$ proposed that the structure of icosahedral quasicrystals can be described as a hierarchical packing of pseudo-Mackay icosahedron (PMI). The PMI is a cluster of 51 atoms, consisting of three concentric atomic shells with icosahedral symmetry. Alternatively, in terms of dense atomic planes, the resulting structure may also be described by quasiperiodic stacking of fivefold, atomically-dense layers along the fivefold axis. Mathernatically, the icosahedral quasiperiodic structure can be generated via an irrational projection from a $6 \mathrm{D}$ periodic hypercubic lattice. The nodes of the lattice are decorated by atomic hypersurfaces (often referred as atomic surfaces). Katz et al. $[15,16]$ chose a set of 
three polyhedra as atomic surfaces properly shaped and localized in 6D space, and investigated the local configurations in $\mathrm{i}-\mathrm{AlCuFe}$ by cell decomposition. The irrational projection from $6 \mathrm{D}$ space defines the 3D structure of icosahedral quasicrystals. The resulting structure is dominated by Bergman clusters (33-atom clusters consisting of two concentric atomic shells) and some additional atomic positions form Mackay clusters that intersect with Bergman clusters [15, 17-19]. In search of a unique description for the model to include all the atomic positions, Duneau [20] showed that the extended Bergman clusters (105-atom and six-shell) are able to cover up to about $98 \%$ atomic positions. More recently, a comprehensive study of atomic clusters in icosahedral F-type quasicrystals concluded that the structures are best described by a network of Bergman clusters connected together by edges rather than the isolated Mackay clusters [21].

As well as the discussions of the bulk structure, quasicrystals have also attracted considerable attention in surface science. On one hand, the study of surface structure is of fundamental interest. The basic question is whether the bulk quasiperiodicity is maintained up to the surface. Furthermore, the resolved surface structure will provide a crucial test for bulk structure models. On the other hand, some unusual and useful properties of quasicrystals are related to surface phenomena. The question becomes whether these physical properties are determined by their surface structure. Answers to such questions will guide more studies of the application of quasicrystalline materials. The surface investigations have focused on icosahedral AI-Pd-Mn and $\mathrm{Al}-\mathrm{Cu}-\mathrm{Fe}$ compounds.

A clean surface in ultrahigh vacuum (UHV) is one of the fundamental requirements for surface science studies. Mainly two methods have been used to prepare clean surfaces for icosahedral quasicrystals. First, the surface can be cleaned by ion sputtering followed 
with annealing. An energetic beam of rare gas ions hits the surface and removes the surface atoms including contaminants, from the surface. Sputtering unavoidably causes a change of surface composition in alloys, due to different sputtering rates for each element. Both icosahedral Al-Pd-Mn and Al-Cu-Fe have an extremely narrow stability domain surrounded by many different phases in the phase diagram. The preferential sputtering of $\mathrm{Al}$ is well known to readily shift the surface composition out of the quasicrystalline region [eg. 22,23]. Besides the composition change, ion bombardment also destroys the atomic order on the surface. Annealing at proper temperature after sputtering is necessary to restore both the surface composition and the surface structure. However, annealing the surface too high will result in the appearance of second phases due to surface segregation and preferential evaporation of surface elements $[24,25,26]$. The procedure of cycles of sputtering and annealing has been successfully developed to obtain reproducible, clean i-AlPdMn and iAlCuFe surfaces $[24,25]$. The other approach used to obtain clean surface in UHV is fracture in-situ. Fracture eliminates the chemical composition change at the surface. However, the surface exposed from cleavage is a non-equilibrium surface. Surfaces of iAlPdMn perpendicular to two and five fold axis produced from cleavage have been studied $[26,27]$.

Various surface analytical techniques have been employed to investigate quasicrystal surfaces. These include, but are not limited to, electron spectroscopy (AES, XPS, UPS), surface diffraction techniques (LEED, XRD) and scanning tunneling microscopy (STM). These techniques provide complementary information regarding the surface structure and surface chemistry. Scanning tunneling microscopy is a fast developing technique in surface science. The operation of the STM is based on the tunneling effect between the surface to be 
investigated and the probing metallic tip [28]. With STM, it is possible to probe the arrangement of individual atoms on surfaces in real space with lateral and vertical resolution of $0.1 \mathrm{~nm}$ and $0.01 \mathrm{~nm}$ respectively. However, the operation of STM at atomic resolution is far from routine and it has certain limitations: STM is not chemically sensitive; and the shape and resolution of a STM scan is greatly influenced by the size, shape and chemistry of the probing tip. Although an atomically sharp tip is achievable, the geometry of the tip is generally not well controlled.

The surface structure of the i-AlPdMn fivefold surface has been investigated intensively with STM. The studies reveal that different preparation methods produce surfaces with different morphologies. The fractured surfaces present a rough, cluster-based topography. The clusters appearing in large-scale scans are formed by the aggregations of smaller elementary clusters of about 0.8 to $1.0 \mathrm{~nm}$ in diameter. Ebert et al. [27] interpret the cluster element as the PMI cluster, the building block for the icosahedral quasicrystals in Boudard and Janot's model [12-14]. The authors conclude that fracture is essentially an intercluster cleavage. For the sputter-annealed sample, the i-AlPdMn fivefold surfaces usually evolve from a rough cluster-like morphology to a step-terrace structure with increasing annealing temperature. The step-terrace surfaces have been subject to numerous analyses regarding two aspects: the stepheights; and the fine structure of the flat terraces [29-35]. A set of stepheights in the ratio of the golden mean $\tau=\frac{\sqrt{5}+1}{2}$, a characteristic number in quasiperiodic structures, has been observed by different authors independently [29-33]. Several research groups have recorded different structural features on the terraces, which may be the result of different tip conditions and/or scanning parameters. Schaub et al. 
[29-32] observed fivefold stars and pentagonal depressions, and they analysed the images in terms of Fibonacci pentagrids. A theoretical model [19], which consists of "geared" layers of Bergman clusters, interprets the sequence of stepheights as well as the related structure on the terraces. By comparing the autocorrelation functions generated from the STM images to those both from the atomic layers and from the distribution of intact PMI clusters in Janot and Boudard's bulk structure model [12-14], Shen et al. [22] concluded that the fine structures they observed on the terraces were PMI clusters rather than atoms. Ledieu and coworkers [35] recently achieved the atomically-resolved STM images from which a tiling based on pentagons with edges of $8.0 \pm 0.3 \AA$ were constructed. The structure matches the atomically-dense plane description of the structure [12], as well as the geometric tiling model [18] based on the 3D tiling $\Gamma^{*}(2 \mathrm{~F})$ projected from a $6 \mathrm{D}$ face-centered hypercubic lattice.

Despite differences in chemical identities and composition, $\mathrm{i}-\mathrm{AlCuFe}$ has a quite similar bulk structure as i-AlPdMn. In this dissertation, we exploit the structure of i-AlCuFe fivefold surfaces prepared by means of cycles of sputtering and annealing. With increasing annealing temperature, our STM studies reveal a similar surface structure progression as that of i-AlPdMn surface, from rough clusters to step-terraces, the latter obtained after annealing at $825 \mathrm{~K}$ and slightly higher temperature. The flat terraces are separated by steps with stepheights of two primary values in the ratio of $\tau$. The observed stepheights are in good agreement with dynamic low energy electron diffraction (LEED) calculations based on bulk structure model. The non-Fibonacci sequence of consecutive stepheights along the fivefold axis implies defects in the layer stacking, which is also indicated by the screw dislocations observed often in the STM images. After we have examined the vertical stacking of terraces 
along the fivefold axis, we continue on to investigate the fine structure on the terraces. In this dissertation, we present the first atomically-resolved STM images on this material. Although i-AlCuFe and i-AlPdMn have very similar bulk structures and surface structures on a large scale, the atomic-scale fine structure on the terraces revealed by STM images for the $\mathrm{i}$-AICuFe fivefold surfaces appear quite different from any recorded patterns for the iAlPdMn surfaces. The flat terraces of $\mathrm{i}-\mathrm{AlCuFe}$ are dominated by flower-like motifs that consist of two rings of bright contrast. Similar flower-patterns can be identified as well in the atomically-dense planes generated from the bulk model [12]. We conclude that the properly sputter-annealed fivefold $\mathrm{i}-\mathrm{AlCuFe}$ surfaces exhibit a quasicrystalline bulkterminated structure. The difference between i-AlPdMn and i-AlCuFe shown in atomic-scale STM images may be due to differences in the surface electronic states for the two materials.

Atomic and molecular adsorption on the quasicrystalline surfaces has become an active area in the field over the past few years [10,36-41]. Adsorbates such as oxygen [36,37], sulphur [10], and $\mathrm{C}_{60}$ [38] on i-AlPdMn have been studied to address issues from several different perspectives. These include the effect of the adsorbate on surface properties, the existence of favourable adsorption sites, and the possibility of forming $2 \mathrm{D}$ quaiscrystalline layers from a single species. In several potential technological applications, quasicrystals may be used as thin film coatings on crystalline materials. This generates interest in studying the structural features at the interface between quasicrystalline and crystalline materials. We approach this interface directly by depositing a thin metallic film on quasicrystalline surface. In this dissertation, we investigate the nucleation and growth of Al thin films on the i-AlCuFe fivefold surface using STM. At very low coverage, we find that $\mathrm{Al}$ adatoms condense into pentagonal "starfish" upon deposition at room temperature. 
Remarkably, all the starfish have uniform size and they adopt the same orientation. This is true within a single terrace and also across different terraces. With increasing coverage, the density of the islands increases so that the pentagonal islands starts to merge with each other. Nevertheless, the pentagonal shape of the individual starfish remains intact. Based on the experimental observations and the available atomic structure model, we identify specific nucleation sites for the aluminum starfish and develop a model for formation of the starfish upon aluminum deposition on the $\mathrm{i}-\mathrm{AlCuFe}$ fivefold surface.

\section{Dissertation Organization}

Three papers are included in this dissertation. The first paper: "Structural aspects of the fivefold quasicrystalline Al-Cu-Fe surface from STM and dynamical LEED studies", is in press with Surface Science. The second paper: "An STM study of the atomic structure of the icosahedral Al-Cu-Fe fivefold surface" is submitted to Physical Review B, Rapid Communication. The third paper "Pseudomorphic starfish: arrangement of extrinsic metal atoms on a quasicrystalline substrate" is submitted to Nature. Following the third paper are general conclusions and appendices that document the published paper "Structural aspects of the three-fold surface of icosahedral Al-Pd-Mn" (appearing in volume 461, issue 1-3 of Surface Science on page L521-L527, 2000), the design as well as the specifications of the aluminum evaporator used in the aluminum deposition study in this dissertation, an extended discussion of the aluminum deposition on the quasicrystalline surface, and the STM database. 


\section{References}

1. N. W. Ashcroft and N. D. Mermin (Solid State Physics, Saunders College Publishing, Fort Worth, 1976)

2. D. Shechtman, I. Blech, D. Gratias, and J. W. Cahn, Phys. Rev. Lett. 53 (1984) 1951

3. C. Janot, Quasicrystals: A Primer, 2nd ed. (Oxford Univeristy Press, Oxford, 1994)

4. International Union of Crystallography, Acta Crystallographia A48 (1992) 922

5. S. J. Poon, Adv. Phys. 41 (1992) 303

6. M. Feuerbacher, C. Metzmacher, M. Wollgarten, K. Urban, B. Baufeld, M. Bartsch and

U. Messerschmidt in New Horizons in Quasicrystals, eds. A. I. Goldman, D. J. Sordelet, P.

A. Thiel and J. M. Dubois (World Scientific, Singapore, 1997) p 103

7. J. M. Dubois, Physica Scripta T49 (1993) 17

8. J. S. Ko, A. J. Gellman, T. A. Lograsso, C. J. Jenks and P. A. Thiel, Surf. Sci. 423 (1999) 243

9. P. G. Gibbons and K. K. Kelton in Physical Properties of Quaiscrystals, eds. M. Cardona, P. Fulde, K. V. Klitzing, R. Merlin, H. -J. Queisser and H. Störmaer (Series Ed. Springer Series in Solid-State Sciences, Vol. 126, Springer-Verlag, Berlin, 1999)

10. A.-P. Tsai, A. Inoue and T. Masumoto, Jpn. J. Appl. Phys. Part 226 (1987) L1505

11. A.-P. Tsai, A. Inoue, Y. Yokoyama and T. Masumoto, Mater. Trans. JIM 31 (1990) 98

12. M. Boudard, M. de Boissieu, C. Janot, G. Heger, C. Beeli, H. -U. Nissen, H. Vincent, R. Ibberson, M. Audier and J. M. Dubois, J. Phys. Condens. Matter 4 (1992) 10149

13. C. Janot and M. de Boissieu, Phys. Rev. Lett. 72 (1994) 1674

14. C. Janot, Phys. Rev. B 53 (1993) 181

15. A. Katz and D. Gratias, J. Non-Crystalline Solids 153 \& 154 (1993) 187 
16. A. Katz and D. Gratias in Proceedings of the 5th International Conference on

Quasicrystals, eds. C. Janot and R. Mosseri (World Scientific, Singapore, 1995) p164

17. V. Elser, Phil. Mag. B 73 (1996) 641

18. P. Kramer, Z. Papadopolos and W. Liebermeister in Proceedings of the 6th International Conference on Quasicrystals, eds. S. Takeuchi and T. Fujiwara (World Scientific, Singapore, 1998) p71

19. G. Kasner, Z. Papadopolos and P. Kramer, Phys. Rev. B 60 (1999) 3899

20. M. Duneau in Proceedings of the 6th International Conference on Quasicrystals, eds. F. Gähler, P. Kramer, H. -R. Trebin and K. Urban (Special Issue of Mater. Sci. Eng. A 294296, Elsevier Science, 2000) p199

21. D. Gratias, F. Puyraimond, M. Quiquandon and A. Katz, Phys. Rev. B 63 (2000) 024202

22. Z. Shen, P. J. Pinhero, T. A. Lograsso, D. W. Delaney, C. J. Jenks and P. A. Thiel, Surf. Sci. 385 (1997) L923

23. C. J. Jenks, J. W. Burnett, D. W. Delaney, T. A. Lograsso and P. A. Thiel, Appl. Surf. Sci. $157(2000) 23$

24. C. J. Jenks, D. Delaney, T. Bloomer, S. -L. Chang, T. Lograsso and P. A. Thiel, Appl. Surf. Sci. 103 (1996) 485

25. F. Schmithüsen, G. Cappello, M. de Boissieu, F. Comin and J. Chevrier, Surf. Sci. 444 (2000) 113

26. Ph. Ebert, F. Kluge, B. Grushko and K. Urban, Phys. Rev. B 60 (1999) 874

27. Ph. Ebert, M. Feuerbacher, N. Tamura, M. Wollgarten and K. Urban, Phys. Rev. Lett. 77 (1996) 3827 
28. C. Bai, Scanning Tunneling Microscopy and Its Applications, 2 nd ed. Springer Series in Surface Sciences, Vol. 32, 2000

29. T. M. Schaub, D. E. Bürgler, H. -J. Güntherodt and J. B. Suck, Phys. Rev: Lett. 73 (1994) 1255

30. T. M. Schaub, D. E. Bürgler, H. -J. Güntherodt, J. B. Suck, Z. Phys. B 96 (1994) 93

31. T. M. Schaub, D. E. Bürgler, H. -J. Güntherodt, J. B. Suck and M. Audier, Appl. Phys. A $61(1995) 491$

32. T. M. Schaub, D. E. Bürgler, C. M. Schmidt and H. -J. Güntherodt, J. Non-Crystl. Solids 205-207 (1996) 748

33. Z. Shen, C. Stoldt, C. Jenks, T. Lograsso, P. A. Thiel, Phys. Rev. B 60 (1999) 14688

34. J. Ledieu, A. Munz, T. Parker, R. McGrath, R. D. Diehl, D. W. Delaney and T. A. Lograsso, Surf. Sci. 433-435 (1999) 666

35. J. Ledieu, R. McGrath, R. D. Diehl, T. A. Lograsso, D. W. Delaney, Z. Papadopolos and G. Kasner, Surf. Sci. Lett. 492 (2001) L729

36. S.-L Chang, W. B. Chin, C.-M. Zhang, C. J. Jenks, P. A. Thiel, Surf. Sci. 337(1/2) (1995) 135-46

37. C. J. Jenks, T. A. Lograsso, P. A. Thiel, J. Am. Chem. Soc. 120(48) (1998) 12668

38. J. Ledieu, C. A. Muryn, G. Thomton, R. D. Diehl, T. A. Lograsso, D. W. Delaney and R. McGrath, Surf. Sci. 472(1-2) (2001) 89

39. M. Shimoda, T. J. Sato, A. P. Tsai and J. Q. Guo, Phys. Rev. B 62 (2000) 11288 40. M. Shimoda, J. Q. Guo, T. J. Sato and A. P. Tsai, Surf. Sci, 482-485(2) (2001) 784 41. B. Bolliger, V. E. Dmitrienko, M. Erbudak, R. Lüscher, H. -U. Nissen and A. R. Kortan, Phys. Rev. B 63 (2001) 052203 


\title{
STRUCTURAL ASPECTS OF THE FIVEFOLD QUASICRYSTALLINE AL-CU-FE SURFACE FROM STM AND DYNAMICAL LEED STUDIES
}

\author{
A paper to be published in Surface Science
}

T. Cai, F. Shi, Z. Shen, M. Gierer, A.I. Goldman, M.J. Kramer, C.J. Jenks, T.A. Lograsso, D.W. Delaney, P.A. Thiel, and M.A. Van Hove

\begin{abstract}
We investigate the atomic structure of the fivefold surface of an icosahedral $\mathrm{Al}-\mathrm{Cu}-\mathrm{Fe}$ alloy, using scanning tunneling microscopy (STM) imaging and a special dynamical low energy-electron diffraction (LEED) method. STM indicates that the step heights adopt (primarily) two values in the ratio of tau, but the spatial distribution of these two values does not follow a Fibonacci sequence, thus breaking the ideal bulk-like quasicrystalline layer stacking order perpendicular to the surface. The appearance of screw dislocations in the STM images is another indication of imperfect quasicrystallinity. On the other hand, the LEED analysis, which was successfully applied to Al-Pd-Mn in a previous study, is equally successful for Al-Cu-Fe. Similar structural features are found for both materials, in particular for interlayer relaxations and surface terminations. Although there is no structural periodicity, there are clear atomic planes in the bulk of the quasicrystal, some of which can be grouped in recurring patterns. The surface tends to form between these grouped layers in both alloys. For Al-Cu-Fe, the step heights measured by STM are consistent with the
\end{abstract}


thicknesses of the grouped layers favored in LEED. These results suggest that the fivefold $\mathrm{Al}-\mathrm{Cu}-\mathrm{Fe}$ surface exhibits a quasicrystalline layering structure, but with stacking defects.

Keywords: Electron-solid interactions, scattering, diffraction; Scanning Tunneling Microscopy (STM); Low Energy Electron Diffraction (LEED); Step formation and bunching; Aluminum; Alloys; Low index single crystal surfaces

\section{Introduction}

The atomic structure at the surfaces of quasicrystals is a matter both of technological and fundamental interest. On the technological side, the highly-ordered but non-periodic bulk structure of these metallic alloys is tied to an unusual combination of physical properties. These properties have already led to some applications as coatings and composites, and may lead to more.[1,2] On the fundamental side, a basic question is how the bulk structure of the three-dimensional quasicrystals (i.e., the icosahedral phases) responds to the twodimensional truncation enforced by a surface. Another is how the bulk and surface structures relate to the macroscopic physical properties.

In the present paper, we employ two techniques to gain insights into the atomic structure at the surface of one particular quasicrystal, icosahedral (i-) Al-Cu-Fe.

The first technique used here is STM, which provides real-space information on some aspects of surface structure. The first report of an STM image of the $5 f$ surface of this particular alloy, i-Al-Cu-Fe, was given by Becker et al., [3]. However, all subsequent work 
on surfaces of icosahedral materials was done with i-Al-Pd-Mn, and in these later studies a very detailed level of analysis emerged. Schaub, et al.[4-6] observed a step-terrace structure that displayed geometric characteristics expected for a bulk-terminated surface, namely, arrangements of features, both lateral and vertical, in Fibonacci sequences. Later, we provided an interpretation of the fine structure on the terraces in terms of the cluster structure of the bulk.[7]. Very recently, Ledieu et al. have analyzed the fine structure in terms of bulk tilings.[8] While these approaches and analyses differ, there is one main conclusion common to all of the three more recent studies (Schaub's, ours, and Ledieu's): The fine structure on the terraces of $\mathrm{i}-\mathrm{Al}-\mathrm{Pd}-\mathrm{Mn}$ is consistent with bulk structural models. Hence, the horizontal structure, i.e. the structure within the surface plane, must be close or identical to that of the bulk.

The step heights measured via STM present a different situation. While Schaub et al. reported only two values of step heights on i-Al-Pd-Mn, we found three; furthermore, we found the frequency of step heights as a function of height to be much different (qualitatively) than did Schaub et al., suggesting a significant difference in the layer-stacking in the two studies. [4-7] Analysis of step height data are important for two reasons: First, they are a test of the vertical 'perfection' of the quasicrystalline surface, i.e. of whether the layers are stacked in a bulk-like sequence; and second, the LEED I-V model predicts specific step heights by predicting separations between favored terminations. Hence, the step heights measured in STM can be cross-checked against the results of the LEED structure model. In this paper, we will report and analyze the step heights on $5 \mathrm{f}$ i-Al-Cu-Fe. 
In the second technique, we exploit the fact that surfaces of the icosahedral quasicrystals display sharp and dense LEED patterns. $[5,6,9-16]$ Dynamical LEED analysis of experimental intensity-voltage (IV) curves can then be used to derive atomic structure, but for quasicrystals a special challenge comes from the non-periodic ordering of the atoms in the bulk. (A number of techniques, including LEED, have confirmed that the fivefold surfaces retain fivefold symmetry.[5-7, 9-20] Hence, it is reasonable to assume that a bulk-terminated surface is a good starting point in the structural analysis.) It is impossible to define a unit cell as in periodic crystals; instead there is an infinite variety of local structures. Therefore an exact dynamical calculation of LEED IV curves for quasicrystals is not feasible, and certain approximations are required to make the analysis possible.

Our previous study of the fivefold surface of $\mathrm{i}-\mathrm{Al}-\mathrm{Pd}-\mathrm{Mn}[12,21]$ showed that a successful dynamical analysis of a fivefold (5f) surface could be achieved. The main modification to the LEED theory was the "average neighborhood approximation," according to which the scattering properties of atoms with similar neighborhoods were assumed to be identical. The success of the approximation was partly due to the fact that a few types of local structures repeat throughout a quasicrystal and thus dominate in the diffraction. For example, more than $50 \%$ of the atoms on the $5 \mathrm{f}$ surfaces of Al-Pd-Mn form pentagons. In addition, the structure of LEED IV curves primarily reflects local structure, mainly because of the short free mean path of the diffracting electrons. [22]

It is reasonable to expect that the same analysis will work for $\mathrm{i}-\mathrm{Al}-\mathrm{Cu}-\mathrm{Fe}$. The bulk structures of $\mathrm{i}-\mathrm{Al}-\mathrm{Cu}-\mathrm{Fe}$ and $\mathrm{i}-\mathrm{Al}-\mathrm{Pd}-\mathrm{Mn}$ are very similar, with minor deviations due mainly 
to differences in composition: the changed composition fractions result primarily in substitution of chemical identities, and secondarily in a small number of added or deleted atoms in some sites. This paper presents the outcome of that analysis, for the $5 f$ surface.

The organization of the paper follows. First, experimental details, both of LEED and STM, are presented in Sec. 2. The theory of the dynamical LEED of quasicrystals is summarized in Sec. 3. In Sec. 4, the experimental STM data, including step height distributions, are presented. In Sec. 5, the dynamical LEED analysis on AI-Cu-Fe is described. In Sec. 6, the results are compared with those for Al-Pd-Mn and conclusions are drawn.

\section{Experimental Description}

Samples were grown, characterized, and prepared as discussed in previous papers. $[23,24]$ The two samples used in STM were adjacent slices from the same single grain, whose composition was determined via inductively coupled plasma-atomic emission spectroscopy (ICP-AES) to be $\mathrm{Al}_{61.5} \mathrm{Cu}_{24.8} \mathrm{Fe}_{13.7}$. The composition of the sample used in

LEED was determined similarly to be $\mathrm{Al}_{63.4} \mathrm{Cu}_{24.0} \mathrm{Fe}_{12.6}$. In both the STM and LEED experiments, we cleaned the sample in ultrahigh vacuum with cycles of ion etching and annealing. In the STM experiments, we used He ions, whereas in the LEED experiments, we used Ar ions. [13] Etching with Ar is known to shift the surface composition far away from the icosahedral region of the phase diagram in the Al-rich quasicrystals. [5, 11, 13, 14, 25-29] Helium was chosen in the STM experiments (done more recently than the LEED 
experiments) to reduce preferential etching of Al.[30]

A fresh sample, introduced from air, was typically cleaned by sputtering for 30 minutes, followed by 30 minutes of annealing, starting at $450 \mathrm{~K}$ and stepping up by $50 \mathrm{~K}$ when significant carbon and oxygen were no longer detected by AES at a given temperature. The sample was heated to a maximum temperature of $875 \mathrm{~K}$.

STM and LEED experiments were performed in three separate ultrahigh vacuum chambers. The two STM chambers were each equipped with an Omicron STM, instrumentation for Auger electron spectroscopy (AES), a mass spectrometer, ion sputtering gun, sample heating capability, and a manifold for introduction of selected pure gases by backfilling. A typical base pressure during STM measurements was 2 to $6 \times 10^{-11}$ Torr. The STM samples were each about $3 \times 4 \mathrm{~mm}^{2}$ in area, and $1.5 \mathrm{~mm}$ thick. Before each STM measurement, the sample was sputtered for 30 minutes with $\mathrm{He}(1.0 \mathrm{KeV}$ beam voltage, 8$10 \mu \mathrm{A}$ from sample to ground with no bias), annealed at the stated temperature for one to two hours, and cooled down to room temperature. The typical tunneling current for the STM measurements was $0.3-0.5 \mathrm{nA}$, and the tunneling voltage was $1.0 \mathrm{~V}$.

In the course of measuring the step heights with STM, we used two different scanners. Comparison revealed that it was important to calibrate the piezoelectrics accurately. We used $\mathrm{Ag}(100)$ as the standard, with known atomic step heights of $2.04 \AA$. Without this in-house calibration, errors up to $40 \%$ would have resulted.

The LEED chamber and measurements have been described previously.[13, 21] 


\section{Experimental STM Data}

After annealing at temperatures lower than $825 \mathrm{~K}$, STM reveals a rough morphology with cluster-like protrusions. These protrusions have different sizes, varying from $1 \mathrm{~nm}$ to $2.5 \mathrm{~nm}$ in diameter (Fig. 1a). Terraces start to appear at about $825 \mathrm{~K}$, though still dotted by clusters (Fig. 1b). At higher annealing temperatures $(850-875 \mathrm{~K})$, a step-terrace morphology predominates (Fig. 1c, 1d). This cluster-to-terrace sequence is similar to the progression of structures that we, and others, have reported already for $5 \mathrm{f}[7,31,32]$ and $3 \mathrm{f}$ [33] surfaces of i-Al-Pd-Mn. Commonly, the well-annealed surfaces exhibit apparent screw dislocations, as shown by the black arrow in Fig. 1e, and by the white arrow in Fig. 1f. It is also common that the large terraces contain broad but shallow pentagonal pits (black arrows in Fig. 1f). These two features--the screw dislocations, and the pentagonal pits--are distinctive, since we never observe screw dislocations and pentagonal pits on the $5 \mathrm{f} \mathrm{Al-Pd-}$ Mn surfaces.

The fine structure on the terraces after high temperature annealing is shown in Fig. lg-h. It consists of many flower-like features that are approximately $18 \AA$ in diameter, (most obvious in Fig. 1h) and of small black holes arranged in Fibonacci pentagrids (most obvious in Fig. 1g). The flowers are presumably the same as the "daisies" noted earlier by Becker, et al..[3] The origin of the fine structure on this surface will be discussed elsewhere. The corrugation in Fig. $1 \mathrm{~g}$ is about $0.5 \AA$ peak-to-peak. (Statistical analysis gives $0.25 \AA$ for the root mean square, and $0.18 \AA$ as the arithmetic mean.) The main point is that the corrugation on the terraces is much smaller than the step heights, and hence does not interfere 
significantly in the following analysis.

We also analyzed the step heights carefully. Determination of individual step heights was problematic, for two reasons. First was the sloping background evident in Fig. 2, which could not be corrected satisfactorily by planing the entire image-either due to STM drift, or to a meandering, large-scale curvature of the surface. Second was the fact that the step height determined from a single line profile varied significantly, depending upon the exact point where the profile cut across the step. Hence, we devised a procedure to obtain a statistical average of heights measured along a continuous step, within localized regions of the image. For background correction, we used standard Omicron software to plane the original image. For individual step heights, we used the Omicron software to construct histograms of pixel intensities in the immediate vicinity of each step. These histograms showed the frequency of $\mathrm{z}$-values versus $\mathrm{z}$ within the local area bridging two neighboring terraces. This resulted in a histogram with two peaks, one corresponding to the lower terrace and the other to the upper terrace. We took the separation of the peaks as the step height across the two terraces. A typical histogram for a single step height measurement spanned roughly $300 \mathrm{~nm}^{2}$. An example of one such histogram is shown in Fig. 3, and corresponds to the pixel height distribution encompassed within the rectangular box of Fig. 1c.

We avoided steps originating obviously from screw dislocations in the analysis of the step height distributions. Steps originating from screw dislocations predominantly displayed heights of $2.5 \AA$.

The entire set of values of step heights is illustrated in the distribution of Fig. 4. We 
see three step heights: $2.5 \AA, 4.0 \AA$ and 6.2-6.6 $\AA$. These values form consecutive ratios of 1.6 and $1.55-1.65$, i.e. close to the golden mean, $\tau=1.618$. The step height of $2.5 \AA$ is found much less frequently than the other two values. For instance, in Fig. 4, we have 30 steps with height of $6.2 \AA$, compared to 5 with a height of $2.4 \AA$.

Line profiles across series of terraces display sequences of step heights such as those illustrated in Fig. 2. Steps are labeled as low $(\mathrm{L})$ and high $(\mathrm{H})$, corresponding to heights of about $4 \AA$ and $6 \AA$, respectively. If the surface were perfectly bulk-terminated, one would expect to see a Fibonacci sequence of $\mathrm{L}$ and $\mathrm{H}$ steps, in which sequences such as L-L-L would be forbidden.[34] The fact that we observe the forbidden L-L-L sequences indicates that the surface cannot be perfectly bulk-terminated; instead, it appears that layers--or, more likely, groups of layers--are stacked imperfectly.

The observation of a relatively high density of screw dislocations on this surface (on the order of 3 dislocations per $200 \mathrm{~nm} \times 200 \mathrm{~nm}$ ) is also consistent with imperfections in the layer stacking sequence. In a regular periodic lattice, as one spirals around a screw dislocation, all layers line up properly again after each turn, preserving a simple sequence of equal step heights. But in a quasicrystal, because of the non-periodic spacings between layers, most layers do not line up correctly when spiraling around a screw dislocation, leading to mismatches and thus stacking errors. For example, if one propagates a step of height $\mathrm{L}$ around a screw dislocation (as in spiral growth), and if that step height is maintained, it will create a sequence of heights L-L-L-..., adding one height $\mathrm{L}$ at each turn. This simple example, if continued, leads to a periodic sequence. 
The exact relationship between screw dislocations and the non-Fibonacci step height sequences is not clear at this stage. Among other things, such a relationship must explain how the screw dislocations generate $2.5 \AA$ step heights in their immediate vicinity, whereas the non-Fibonacci sequences include 4.0 and 6.2-6.6 $\AA$ steps (Fig. 2); for example, a $2.5 \AA$ step may join another step and increase or decrease its height by $2.5 \AA$ (Fig. le). It is nonetheless true that the screw dislocations and the non-Fibonacci step heights are both manifestations of imperfect quasicrystalline stacking, such as locally periodic stacking.

Periodic sequences of interlayer spacings have in fact been observed by highresolution transmission electron microscopy (TEM) in defect areas of bulk i-Al-Cu-Fe samples. Similar periodic regions have been observed by TEM in the types of samples we use, and are associated with strain fields. Strain fields arise because the samples are first prepared via liquid-assisted growth, then hot-isostatically-pressed to reduce porosity, and finally annealed again to reduce strain from the pressing. Strain fields become particularly abundant after the hot isostatic-pressing step, as shown by comparing the TEM images of Fig. $5 \mathrm{a}$ and $5 \mathrm{~b}$. Fig. $5 \mathrm{a}$ is a TEM image of an as-grown sample, whereas Fig. $5 \mathrm{~b}$ shows a sample immediately after pressing. The strain fields are reduced considerably, although not eliminated, after the final annealing. Fig. $5 \mathrm{c}$ is a TEM image after the final treatment, showing a region that is locally perfect. We speculate that remnant strain fields from the hot-pressing procedure may play a role in the imperfections at our surfaces. 


\section{Sketch of Dynamical LEED Theory for Quasicrystalline Surfaces}

In spite of their non-periodicity, quasicrystals often produce LEED patterns with a set of well-defined spots, as shown in Fig. 6. The LEED patterns can be even sharper and clearer than for normal crystals; quasicrystals are thus structurally very different from amorphous materials. The sharp LEED pattern is evidence that quasicrystals are a class of ordered materials with particular long-range order and orientation symmetries; in fact they can be described with self-similar models (i.e. as structures that can be scaled up by constant factors to yield similar structures at different length scales). It has been proven in many studies that the Fourier transform of a quasicrystal structure is well ordered in reciprocal space, although again not periodic.[34] In LEED, the Fourier transform corresponds to single scattering theory. Just as with periodic crystals, multiple scattering does not change the LEED pattern in reciprocal lattice, but modifies the LEED spot intensities. The LEED IV curves for these patterns have the same qualitative appearance as for a normal crystal, and are illustrated in Fig. 7 and Fig. 8, where diffraction spot intensity is plotted as a function of momentum transfer parallel to the surface, $\mathrm{Dk}_{\|}$.

As a starting point for the structural LEED analysis, we need a source of bulk atomic coordinates with icosahedral symmetry. Quasicrystals are most conveniently represented as a periodic bcc lattice in 6 dimensions. The "atoms" in that lattice are replaced by atomic hypersurfaces. Tricontahedral atomic hypersurfaces, instead of the usual spherical ones, are used in our models for $\mathrm{Al}-\mathrm{Cu}-\mathrm{Fe}$ and $\mathrm{Al}-\mathrm{Pd}-\mathrm{Mn}$, in order to yield reasonable bond lengths. [35] The three-dimensional structure is generated as a particular projection from that six- 
dimensional bcc lattice into a 3-dimensional space, and the two-dimensional surface is obtained by terminating the three-dimensional lattice. The bulk structure of $\mathrm{Al}-\mathrm{Cu}-\mathrm{Fe}$ is determined by x-ray and neutron diffraction, which fix the atomic hypersurfaces. Once the 3dimensional bulk structure of $\mathrm{Al}-\mathrm{Cu}-\mathrm{Fe}$ is determined, a $5 \mathrm{f}$ surface is obtained by rotating the bulk structure so that the surface plane is perpendicular to the $5 \mathrm{f}$ axis.

The resulting atomic structure along the surface normal shows well-defined and separated atomic planes, with one, two or three chemical types of atoms in each plane. (We shall use the term "plane" for sets of coplanar atoms and "layer" for more general sets of atoms, including groups of atomic "planes", as in "composite layers"). The interplanar spacings vary systematically according to Fibonacci sequences and the Golden mean. As a result of the non-periodicity, each plane is different. If we assume that the surface terminates along such atomic planes, there is, strictly speaking, an infinite number of inequivalent terminations. Within each atomic plane, although there is no repeating unit cell, one finds repeating local $5 f$ rings throughout the plane. The atomic density of different planes varies strongly in the range from 100 to 800 atoms in an area of $100 \AA \times 100 \AA$. The smallest size of the local rings varies with the atomic density. These planes are found to belong to subsets of planes with very similar compositions, atomic densities and geometry. Thus, the infinite variety of planes actually has a relatively small number of distinct types of structure, and we shall exploit this property.

The major difficulty in the LEED analysis of quasicrystals comes from multiple scattering. In a single scattering LEED theory, which assumes that all electrons are scattered 
only once before they leave the surface, the outgoing wave amplitude from an atom depends only on the chemical identity and the position of the atom. In dynamical LEED, however, the outgoing wave amplitude from an atom is composed not only of that directly scattered wave, but also of waves that have undergone other scattering events within the surface. The latter events depend not only on the chemical identity and position of the scatterer, but also on the environment of the scatterer. In principle, the environment of every atom is different from that of every other atom, measured on an infinitely large scale. Therefore, the total wave amplitude of outgoing electrons from any given atom is different from that from all other atoms. In this sense, there are an infinite number of different atoms that have to be taken account in a dynamical LEED analysis of quasicrystals. This is fundamentally different from the dynamical LEED theory of periodic crystals, in which the number of different atoms is finite, due to the repeating unit cells.

As a result, certain approximations have to be made in a realistic dynamical LEED analysis of quasicrystals. In this paper, we apply efficient approximations that were tested in a previous LEED analysis of an i-Al-Pd-Mn surface; [21] these are sketched in the following. The basic idea is that the local environments of atoms tend to have only a few basic structural arrangements, as described above. Due to the limited mean free path of propagating electrons within the surface, the LEED IV curves are determined primarily by the scattering within such local environments. The different approximations depend on how we treat the similar local environments in a quasicrystal lattice.

More specifically, the approximations in our theory take into account that the atoms 
are explicitly arranged in atomic planes parallel to the $5 \mathrm{f}$ surface in a bulk terminated quasicrystal. The atoms within a given atomic plane are typically evenly distributed in space. The atomic density measured per unit area is uniform within a plane, but fluctuates from plane to plane, in the range $0.01-0.13$ atoms $/ \AA^{2}$. The "effective" atomic scattering properties (including all multiple scattering events) are more alike within an atomic plane than between different atomic planes, as the average scattering properties of atoms in an atomic plane depend largely on the composition and the atomic density of that plane. Therefore, as an approximation, we assume that all atoms within a certain atomic plane are equivalent, but different from atoms in other planes.

This approximation, in the single scattering theory, implies that the scattering properties of an atom are averaged over chemical identities. It leads to the average t-matrix approximation (ATA), [36] [37] [38] according to which the scattering matrices t of the individual atoms within one plane are replaced by an averaged scattering t-matrix:

$$
\langle t\rangle=c_{A l} t_{A l}+c_{C u} t_{C u}+c_{F e} t_{F e}
$$

where $C_{i}$ are the relative concentrations within the plane.

Next, as an approximation in the multiple scattering LEED theory, the variable environments of the atoms in a particular plane are replaced by a fixed, simplified average geometry, referred to as "average neighborhood approximation" (ANA). More specifically, the final wave amplitude from an atom, with all multiple scattering events considered, is replaced by an averaged wave amplitude over all atoms in the plane. The ATA is applied before the wave amplitude is averaged. For more details, see Ref. [21]. 
With these approximations, we can perform the calculation with a relatively small number of atoms, equivalent to the number of atomic planes, with different plane-dependent scattering properties. If we take atomic planes as deep as $10 \AA$ into account, we obtain about 12 planes, i.e. 12 atoms with different scattering properties. The incident beam is damped into the surface due to inelastic scattering events, so that the contributions of deeper atoms do not influence the IV curves significantly. The calculation of the averaged propagator matrix $\langle G\rangle$, which describes electron propagation within and between closely-spaced planes, is performed by averaging in an area of $100 \AA \times 100 \AA$. The averaged wave amplitude depends strongly on the atomic density of the plane. For a plane with high atomic density, the averaged amplitude is affected by multiple scattering events within the plane more than that between planes. Inversely, for a plane with low atomic density, it is affected more by the multiple scattering events between atomic planes than by that within the plane.

The ANA, in which all atoms in a particular atomic plane have the same averaged effective scattering properties, can be improved in several respects. First, it is possible to use the ANA without the ATA. In this case, a single coplanar atomic plane is divided into several mono-atomic planes such that each has only one chemical species. All atoms in one mono-atomic plane are then assumed to have the same scattered wave amplitude. The computation time increases because one has to deal with more mono-atomic planes, i.e. more atoms in the calculation. It has been demonstrated for Al-Pd-Mn that most IV curves are little affected by the use of the ATA: thus one can safely apply ATA and gain computational time. The ANA may be further improved if one divides an atomic plane into 
several subplanes in which the atoms in each subplane have exactly the same local environment within and out of the plane. For example, one can assume that the local environments of two atoms are similar only if the nearest-neighbor distances and the number of nearest-neighbors are equal. All the atoms are thus sorted into a few classes of atoms, each with the same local neighborhoods. Notice that the number of different atoms increases sharply if the size of the local neighborhood increases. In the LEED calculation for Al-PdMn 5 f surfaces, it was shown that the IV curves are also very similar under this approximation. This at least shows that the ANA is reliable for the $5 f$ quasicrystal surfaces. However, the approximation was found to be less successful for the surfaces of Al-Pd-Mn with lower symmetry, such as the two- and three-fold surfaces. [39]

\section{LEED Structural Analysis}

Some of the experimental LEED IV curves are shown in Fig. 7 and Fig. 8. Normal incidence is established by optimizing the agreement between curves for different, but symmetry-equivalent, diffraction spots, as shown in Fig. 7. Each curve shown in Fig. 8 is a symmetry-averaged composite, normalized to approximately the same value. Different curves represent different sample treatments. We varied sample treatments in order to check the robustness of the IV data. In all, we reproduced the complete set of IV curves 9 times. We conclude that the IV curves are not sensitive to sample preparation conditions, within the range of sputtering conditions (15-30 minutes with $\left.\mathrm{Ar}^{+}\right)$and annealing conditions (1-2 hours at $800-850 \mathrm{~K}$ ) tested. This is similar to our previous findings for $5 \mathrm{f} \mathrm{i}-\mathrm{Al}-\mathrm{Pd}-\mathrm{Mn}$, 
where the experimental IV curves were also found to be very robust.

In the LEED analysis of the $5 \mathrm{f} \mathrm{Al-Cu-Fe} \mathrm{surface,} \mathrm{we} \mathrm{first} \mathrm{explored} \mathrm{individual} \mathrm{bulk}$ terminations, with possible relaxations of the top few interplanar spacings. Later we also considered combinations of terminations. For the calculation, we used relativistic phase shifts of $\mathrm{Al}, \mathrm{Cu}$ and $\mathrm{Fe}$, which have also been used in the LEED analysis of the (110) surface of crystalline $\mathrm{b}-\mathrm{Al}_{\mathrm{I}-\mathrm{x}}(\mathrm{CuFe})_{\mathrm{x}}$ with a similar composition. [40] Thermal effects were included through the usual Debye-Waller factor. The Pendry R- factor was used for comparison between theory and experiment.

We thus started by analyzing a large set of individual terminations of the $5 \mathrm{f} \mathrm{Al}-\mathrm{Cu}-\mathrm{Fe}$ surfaces, namely those that exist within a rectangular box with surface dimensions of $100 \AA x$ $100 \AA$ and a depth of $50 \AA$. Those terminations run through all the atomic planes along the surface normal in the entire depth of the box. For each termination, the top 12 atomic planes are chosen as a composite layer for LEED calculation. At first, in a rough search, we only allowed the relaxation of the topmost interplanar spacing using a grid search, i.e. the R-factor was calculated on a grid of points in the range from -0.3 to $0.3 \AA$.

The resulting R-factors for all the terminations that we examined are plotted in Fig. 9. It is seen that the R-factor varies from about 0.5 to 0.9 for different terminations. The Rfactor drops if more planes are allowed to relax, but it is found that the change in $\mathrm{R}$-factors is much smaller than the fluctuations between R-factors for different terminations. So the Rfactors shown in Fig. 9 are reliable as a basis for further analysis. It is also interesting to find that the different terminations yield very similar optimized structural parameters. 
Next, we focused on the most promising surface terminations as given by the Rfactors shown in Fig. 9. We interpret the "better" terminations of Fig. 9 to be all present on the real surface, forming an array of terraces separated by steps of variable height.

The favored step heights can be understood in terms of groups of closely-spaced planes. Even though there is no periodic repetition in the quasicrystalline bulk structure, certain groups of planes occur frequently (even if not identically). We recognize three main sets of grouped planes, each group containing 3,5 or 9 atomic planes (they can be identified in Fig. 9, while the 5- and 9-plane cases are also illustrated in Figure 10; note that there sometimes exist additional planes of very low atomic density between these planes, resulting in 11-plane groups, for instance). Within each group, the distance between successive atomic planes is smaller than $0.78 \AA$. The different groups are mutually separated by at least a distance of 1.5-1.6 $\AA$. The two large groups with 5 and 9 planes share some common features. Each group has an approximate central symmetry around the middle plane. Both outer surfaces of such a group have two planes separated by only $0.48 \AA$; these planes have the highest combined atomic density.

It is found that the surface tends to form between these grouped planes, i.e. by splitting through the relatively large spacings of $1.5-1.6 \AA$, and thus exposing the closelyspaced pairs of planes. Since the outermost plane within these pairs has a high aluminum concentration and the other an $\mathrm{Al}: \mathrm{Cu}$ ratio of about 50:50, these pairs are enriched in aluminum compared to the bulk average. Their combined surface atomic density is close to that of the densely-packed $\mathrm{Al}$ (111) crystalline surface. The step heights between terraces 
with such terminations are obtained by measuring the distances between two kinds of terminations, which are $3.99 \AA$ for 5-plane steps (Fig. 10 shows one such step) and $6.47 \AA$ for 9-plane steps; 3-plane steps give $2.40 \AA$ heights. (Spacing relaxations, if identical for the terminations involved, do not change these distances).

The next stage in our LEED analysis mixes different terminations. In a full LEED analysis of quasicrystals, one can explicitly take into account the coexistence of terraces with different types of terminations. Since they are all assumed to contribute to the LEED IV curves, one needs to mix their contributions. Assuming that the area of each terrace is large compared to the coherence width of the electron beam (which is justified by the STM observations in Section 3), then only the reflected intensities from different terraces need to be mixed, as opposed to reflected amplitudes. As shown both in the LEED analysis and in the STM data (Sec. 3), the frequencies of the three kinds of terminations are different. To reduce the number of fit parameters, we take into account that the terminations in 5-plane groups appear to have the better R-factors, and mix only this kind of termination. Since they have essentially the same structure perpendicular to the surface, the number of fitparameters can be reduced to a few, representing the number of interplanar spacings that one wishes to fit.

We thus mixed the 6 terminations that yielded the best $R$-factors (indicated by the short arrows in Fig. 9) and gave them equal weights. We also allowed relaxation of the top 5 interplanar spacings. This was done by the linear LEED approximation.[41] In this very efficient approach, the wave fields are calculated for one reference and several trial 
structures. Then the Powell optimization scheme is applied to optimize the structure. The linear LEED approximation is valid for this material if the deviations between reference and trial structures are smaller than about $0.2 \AA$ (which was always the case in our analysis).

As a result, the R-factor could be reduced to 0.39 , which we take as our optimum fit. The comparison between the experimental and theoretical IV curves for this best-fit structure is shown in Fig. 11, and the structure itself in Fig. 12 (again, this represents an average best-fit structure for the 5-plane terminations). The topmost interplanar spacing contracts from the bulk value by $0.10 \pm 0.08 \AA$, going from $0.48 \AA$ to $0.38 \AA$, while the second interplanar spacing expands, the third interplanar spacing contracts and the deeper spacings are close to bulk values, cf. Fig. 12. Since the third plane has a low atomic density, the error bar for the second and third interplanar spacings is large (comparable to the changes from the bulk values).

We assumed the bulk composition for each layer, since the earlier work with Al-PdMn showed little sensitivity of LEED to these compositions.[21] Thus, on average, the bulk composition of the outermost atomic plane is $90 \% \mathrm{Al}$ and $10 \% \mathrm{Fe}$ atoms, while the second atomic plane contains $45 \% \mathrm{Al}, 45 \% \mathrm{Cu}$ and $10 \% \mathrm{Fe}$ atoms. The average lateral density of the two top planes taken together is 0.14 atoms $/ \AA^{2}$, a value very close to that of a single plane of Al(111), 0.141 atoms $/ \AA^{2}$. This indicates a very densely packed surface double-layer. We notice that the optimized structure for a single termination is exactly the same as that for mixed terminations. This again indicates that the selected terminations are very similar in structure. 
From Fig. 9 it is to be noted that some 9- and 3-plane terminations give R-factors that are not much worse than those for 5-plane terminations. While the LEED analysis cannot be used to state that these terminations are also present on the surface or with what relative abundance, at least the LEED analysis is consistent with the presence of some 9- and 3-plane terminations on the surface: the LEED results are therefore also compatible with the STM data, which indicate the presence of several step heights.

Finally, we note that other authors have suggested that three step heights may be a normal situation on 5 fold surfaces of icosahedral quasicrystals. Fradkin $[42,43]$ has shown that three step heights are to be expected on supercooled quasicrystalline samples along the fivefold axis. The relative population of these three step heights depends upon the extent of supercooling, i.e. the extent of deviation from equilibrium. At the exact points where one population disappears, the ratio of the other two populations should be $\tau=1.61 \ldots$ In Fig. 4, the $2.4 \AA$ population is very small, and in the context of the Fradkin model, one might think that it is on the verge of disappearing. The ratio of the three populations is about $9 \%: 37 \%: 54 \%$, i.e. the two largest populations are in the ratio of about 1.5 . While this value is not exactly $\tau$, it is close enough to be qualitatively in accord with the hypothesis of Fradkin. $[42,43]$

Another prediction has been made recently by Gratias, et al., [44] who, on the basis of the specific structural model for $\mathrm{i}-\mathrm{Al}-\mathrm{Cu}-\mathrm{Fe}$, forecast that steps should adopt three values of heights with predictable frequencies. These heights (frequencies) are, relative to the longest step, $\mathrm{L}: \mathrm{L}(31 \%) ; \mathrm{L} / \tau(51 \%) ;$ and $\mathrm{L} / \tau^{2}(19 \%)$, i.e. the two largest populations should 
be in the ratio of 1.65 . Again, we see that our experimental value of 1.5 is only slightly lower than expected. Also, our experimental values of the step heights $(2.5,4.0$, and 6.2-6.6 $\AA$ ) follow the predicted ratio of $\mathrm{L}, \mathrm{L} / \tau$, and $\mathrm{L} / \tau^{2}$ exactly.

\section{Conclusions}

We have analyzed the atomic-scale structure of the $5 \mathrm{f}$ surface of an $\mathrm{Al}-\mathrm{Cu}-\mathrm{Fe}$ quasicrystal by STM imaging and a special dynamical LEED analysis. Basically, the surface is bulk-terminated with presumably a bulk-like layer-dependent composition. Our analysis finds that the surface tends to form between different groups of closely-spaced planes, i.e. by splitting the quasicrystal through the larger interplanar spacings that separate those groups. In particular, we find that those grouped planes can be sorted into three sets, with 3 , 5 and 9 planes, respectively. The interplanar spacings are the same for the same set of grouped planes. The 5-plane and 9-plane groups are similar in that the top pair of planes have the highest combined atomic density, about 0.14 atoms $/ \AA^{2}$--very close to that of the $\mathrm{Al}$ (111) surface--and are rich in Al.

The sets of 3,5 , and 9 planes correspond to step heights of $2.47,3.99$, and $6.47 \AA$, respectively. The step height frequencies seen in STM hence favor the 9-plane steps, then the 5-plane steps and finally the 3-plane steps (Fig. 4). LEED does not measure step heights directly: the R-factors favor 5-plane terminations, then 9- and 3-plane terminations. The LEED R-factors contain information both on the quality of fit of individual coexisting terminations, and on the frequency of occurrence of the different terminations; however, it is 
not clear how one could extract these two aspects separately from the data. Nonetheless, the fact that LEED and STM favor the same sets of step heights indicates that the two techniques are in overall agreement on the types of terminations present at the surface. The measured distribution is also roughly consistent with the predictions of Fradkin,[42, 43] and of Gratias, et al.[44]

The sequence of step heights in STM (Fig. 2) suggests that there are defects in layer stacking, relative to a perfect bulk quasicrystalline termination. LEED shows that groups of planes are separated by distances of $1.4-1.6 \AA$ with no atoms in between, as illustrated in Fig. 10. We speculate that "mistakes" in the quasicrystalline lattice, normal to the surface, occur between these groups of planes. In other words, we hypothesize that quasicrystalline order is maintained well within favored groups of planes, where atoms interconnect densely throughout the structure, but less well between groups of planes. Perhaps these mistakes occur as the surface regrows after ion bombardment. Or perhaps they are engendered by defects in the bulk structure incorporated during sample preparation (Fig. 5). In either case, this hypothesis provides some understanding for the observation that there is stronger evidence for defects perpendicular to the surface than for defects within, or very near to, the surface plane.

Finally, it is interesting to compare the present results for $5 \mathrm{f}$ - $\mathrm{Al}-\mathrm{Cu}-\mathrm{Fe}$, and those reported previously for $5 \mathrm{f} \mathrm{i}-\mathrm{Al}-\mathrm{Pd}-\mathrm{Mn}$. The main difference is that the $\mathrm{i}-\mathrm{Al}-\mathrm{Cu}-\mathrm{Fe}$ surface contains screw dislocations and pentagonal pits, neither of which has been observed on i-Al$\mathrm{Pd}-\mathrm{Mn}$, in our laboratory and under analogous conditions. Our general impression is that the 
i-Al-Cu-Fe surface contains more defects, perhaps a remnant of the hot-pressing step which is a part of sample preparation, as discussed in Section 3. On the other hand, there are several similarities between the two types of surfaces. Both have been analyzed successfully by dynamical LEED theory. Similar R-factors are obtained for both materials. The preferred terminations, atomic densities, and chemical compositions are similar. As a result, the surface in both cases is aluminum-rich. The implied step heights are also very comparable. And the outermost interplanar spacing is contracted on both materials by $0.1 \AA$, leaving deeper spacings bulk-like within the error bars. Further discussion of geometric aspects of these surface structures can be found in ref. [21], given that the two materials are structurally so similar.

\section{Acknowledgments}

We thank M. Quiquadon for providing useful information. This work was supported by the Director, Office of Science, Office of Basic Energy Sciences, Materials Sciences Division of the U.S. Department of Energy under Contract Nos. DE-AC03-76SF00098 and W-405-Eng-82.

\section{References}

1. P. A. Thiel, J. M. Dubois, Materials Today 2 (1999) 3.

2. Z. M. Stadnik, Physical Properties of Quasicrystals, M. Cardona, P. Fulde, K. v. Klitzing, R. Merlin, H.-J. Queisser, H. Störmer (Series Ed.) Springer Series in Solid-State Sciences, 
Vol. 126, Springer-Verlag, Berlin, 1999.

3. R. S. Becker, A. R. Kortan, F. A. Thiel, H. S. Chen, J. Vac. Sci. Technol. B 9 (1991) 867.

4. T. M. Schaub, D. E. Bürgler, H.-J. Güntherodt, J. B. Suck, Phys. Rev. Lett. 73 (1994) 1255.

5. T. M. Schaub, D. E. Bürgler, H.-J. Güntherodt, J. B. Suck, M. Audier, Appl. Phys. A 61 (1995) 491.

6. T. M. Schaub, D. E. Bürgler, H.-J. Güntherodt, J.-B. Suck, Z. Phys. B 96 (1994) 93.

7. Z. Shen, C. Stoldt, C. Jenks, T. Lograsso, P. A. Thiel, Phys. Rev. B 60 (1999) 14688.

8. J. Ledieu, R. McGrath, R.D. Diehl, T.A. Lograsso, D.W. Delaney, Z. Papadopolos, G.

Kasner, Surface Sci. Lett. (2001) in press.

9. T. M. Schaub, D. E. Bürgler, H.-J. Güntherodt, J. B. Suck, M. Audier, "Fivefold symmetric LEED patterns measured on icosahdral Al68Pd23Mn9," in: C. Janot, R. Mosseri (Ed.) Proceedings of the 5th International Conference on Quasicrystals (ICQ5), Vol. World Scientific, Singapore, 1995, p. 132.

10. X. Wu, S. W. Kycia, C. G. Olson, P. J. Benning, A. I. Goldman, D. W. Lynch, Phys. Rev. Lett. 75 (1995) 4540.

11. S.-L. Chang, W. B. Chin, C.-M. Zhang, C. J. Jenks, P. A. Thiel, Surf. Sci. 337 (1995) 135.

12. M. Gierer, M. A. Van Hove, A. I. Goldman, Z. Shen, S.-L. Chang, C. J. Jenks, C.-M. Zhang, P. A. Thiel, Phys. Rev. Lett. 78 (1997) 467.

13. Z. Shen, P. J. Pinhero, T. A. Lograsso, D. W. Delaney, C. J. Jenks, P. A. Thiel, Surface 
Sci. 385 (1997) L923.

14. J. Ledieu, A. Munz, T. Parker, R. McGrath, R. D. Diehl, D. W. Delaney, T. A. Lograsso, Surface Science 433-435 (1999) 666.

15. G. Cappello, A. Dechelette, F. Schmithüsen, J. Chevrier, F. Comin, A. Stierle, V. Formoso, M. de Boissieu, T. Lograsso, C. Jenks, D. Delaney, "Characterization and properties of the AlPdMn 5 surface," in: J. M. Dubois, P. A. Thiel, A. P. Tsai, K. Urban (Ed.) MRS Proceedings: Quasicrystals, Materials Research Society Symposium Proceedings Vol. 553, MRS, Boston, 1999, p. 243.

16. Z. Shen, W. Raberg, M. Heinzig, C. J. Jenks, V. Fournée, M. A. V. Hove, T. A.

Lograsso, D. Delaney, T. Cai, P. C. Canfield, I. R. Fisher, A. I. Goldman, M. J. Kramer, P. A. Thiel, Surface Science 450 (2000) 1.

17. M. Erbudak, H.-U. Nissen, E. Wetli, M. Hochstrasser, S. Ritsch, Phys. Rev. Lett. 72 (1994) 3037.

18. D. Naumovic, P. Aebi, L. Schlapbach, C. Beeli, "A real-space and chemically-sensitive study of the i-AlPdMn pentagonal surface," in: A. I. Goldman, D. J. Sordelet, P. A. Thiel, J. M. Dubois (Ed.) New Horizons in Quasicrystals: Research and Applications, Vol. World Scientific, Singapore, 1997, p. 86.

19. D. Naumovic, P. Aebi, L. Schlapbach, C. Beeli, T. A. Lograsso, D. W. Delaney, "Study of the 5-fold and 2-fold i-AlPdMn surfaces by full-hemispherical x-ray photoelectron diffraction," in: S. Takeuchi, T. Fujiwara (Ed.) Proceedings of the 6th International Conference on Quasicrystals (ICQ6), Vol. World Scientific, Singapore, 1998, p. 749. 
20. R. Bastasz, C. J. Jenks, T. A. Lograsso, A. R. Ross, P. A. Thiel, J. A. Whaley, "LowEnergy Ion Scattering Measurements from an Al-Pd-Mn Quasicrystal," in: E. Belin-Ferré, P. A. Thiel, K. Urban, A.-P. Tsai (Ed.) MRS Conference Proceedings: Quasicrystals, Vol. MRS, Warrendale, NJ, 2001,

21. M. Gierer, M. A. Van Hove, A. I. Goldman, Z. Shen, S.-L. Chang, P. J. Pinhero, C. J. Jenks, J. W. Anderegg, C.-M. Zhang, P. A. Thiel, Phys. Rev. B 57 (1998) 7628.

22. D. K. Saldin, J. B. Pendry, M. A. Van Hove, G. A. Somorjai, Phys. Rev. B31 (1985) 1216.

23. C. J. Jenks, P. J. Pinhero, Z. Shen, T. A. Lograsso, D. W. Delaney, T. E. Bloomer, S.-L. Chang, C.-M. Zhang, J. W. Anderegg, A. H. M. Z. Islam, A. I. Goldman, P. A. Thiel, "Preparation of icosahedral AIPdMn and AlCuFe samples for LEED studies," in: S. Takeuchi, T. Fujiwara (Ed.) Proceedings of the 6th International Conference on Quasicrystals (ICQ6), Vol. World Scientific, Singapore, 1998, p. 741.

24. C. J. Jenks, D. W. Delaney, T. E. Bloomer, S.-L. Chang, T. A. Lograsso, Z. Shen, C.-M. Zhang, P. A. Thiel, Appl. Surf. Sci. 103 (1996) 485.

25. S.-L. Chang, J. W. Anderegg, P. A. Thiel, J. Non-cryst. Solids 195 (1996) 95. 26. S. Suzuki, Y. Waseda, N. Tamura, K. Urban, Scripta Materialia 35 (1996) 891. 27. D. Rouxel, M. Gavatz, P. Pigeat, B. Weber, P. Plaindoux, "Auger electron microprobe analysis of surface of $\mathrm{Al}_{62} \mathrm{Cu}_{25.5} \mathrm{Fe}_{12.5}$ quasicrystal. First steps of oxidation.," in: A. I. Goldman, D. J. Sordelet, P. A. Thiel, J. M. Dubois (Ed.) New Horizons in Quasicrystals: Research and Applications, Vol. World Scientific, Singapore, 1997, p. 173. 
28. D. Naumovic, P. Aebi, C. Beeli, L. Schlapbach, Surf. Sci. 433-435 (1999) 302.

29. F. Schmithüsen, G. Cappello, M. De Boissieu, M. Boudard, F. Comin, J. Chevrier, Surface sci. 444 (2000) 113.

30. C. J. Jenks, J. W. Burnett, D. W. Delaney, T. A. Lograsso, P. A. Thiel, Applied Surface Science 157 (2000) 23.

31. J. Ledieu, A. W. Munz, T. M. Parker, R. McGrath, R. D. Diehl, D. W. Delaney, T. A. Lograsso, "Clustered, terraced, and mixed surface phases of the $\mathrm{Al}_{70} \mathrm{Pd}_{21} \mathrm{Mn}_{9}$ quasicrystal," in: J. M. Dubois, P. A. Thiel, A.-P. Tsai, K. Urban (Ed.) MRS Proceedings: Quasicrystals, Materials Research Society Symposium Proceedings Vol. 553, Materials Research Society, Warrendale, Pennsylvania, 1999, p. 237.

32. G. Cappello, Ph.D. Thesis, Université Joseph Fourier--Grenoble I (2000).

33. D. Rouxel, T. Cai, C. J. Jenks, T. Lograsso, A. Ross, P. A. Thiel, Surface Sci. 461 (2000) L521.

34. C. Janot, Quasicrystals: A Primer, C. J. Humphreys, P. B. Hirsch, N. F. Mott, R. J. Brook (Series Ed.) Monographs on the Physics and Chemistry of Materials, Vol. 48, Clarendon Press, Oxford, 1992.

35. A. Katz, D. Gratias, J. Non-Cryst. Solids 153-154 (1993) 187.

36. Y. Gauthier, Surf. Rev. Lett. 3 (1996) 1663.

37. F. Jona, K. O. Legg, H. D. Shih, D. W. Jepsen, P. M. Marcus, Phys. Rev. Lett. 40 (1978) 1466.

38. P. J. Rous, Surf. Sci. 244 (1991) L137. 
39. M. Gierer, F. Shi, Z. Shen, P. Thiel, C. Jenks, A. Goldman, M. A. Van Hove, (1998) unpublished results.

40. F. Shi, Z. Shen, D. W. Delaney, A. I. Goldman, C. J. Jenks, M. J. Kramer, T. Lograsso, P. A. Thiel, M. A. Van Hove, Surface Science 411 (1998) 86.

41. A. Wander, J. B. Pendry, M. A. Van Hove, Phys. Rev. B 46 (1992) 9847.

42. M. A. Fradkin, "Structure selection and generation of phasons at the growing surface of quasicrystal," in: G. Chapuis, W. Paciorek (Ed.) Aperiodic Crystals, Vol. World Scientific, Singapore, 1995,

43. M. A. Fradkin, JETP Letters 69 (1999) 570.

44. D. Gratias, F. Puyraimond, M. Quiquandon, A. Katz, Phys. Rev. B 63 (2001) 024202. 


\section{Figure Captions}

Figure 1. Micrographs of the 5 Al-Cu-Fe surface, after different thermal treatments and at different magnifications. (a) $\mathrm{T}<825 \mathrm{~K}, 30 \times 30 \mathrm{~nm}^{2}$ (b) $\mathrm{T} \approx 825 \mathrm{~K}, 50 \times 50 \mathrm{~nm}^{2}$ (c) $\mathrm{T}>$ $825 \mathrm{~K}, 100 \times 100 \mathrm{~nm}^{2}$ (d) $\mathrm{T}>825 \mathrm{~K}, 200 \times 200 \mathrm{~nm}^{2}$ (e) $\mathrm{T}>825 \mathrm{~K}, 100 \times 100 \mathrm{~nm}^{2}$. Black arrow shows the origin of a screw dislocation. (f) $\mathrm{T}>825 \mathrm{~K}, 100 \times 100 \mathrm{~nm}^{2}$. White arrow points to a screw dislocation; black arrows point to the pentagonal facets. (g) $\mathrm{T} \approx 825 \mathrm{~K}$, $50 \times 50 \mathrm{~nm}^{2}$. (h) $\mathrm{T} \approx 825 \mathrm{~K}, 15 \times 15 \mathrm{~nm}^{2}$. The circle outlines a flower.

Figure 2. Line profiles across consecutive terraces, from the data of Figs. 1c-d. The labels (i)-(iii) indicate the specific profiles in Figs. 1c-d. Steps are labelled as H, high (6.2-6.6 $\AA)$ or $\mathrm{L}, \operatorname{low}(4.0 \AA)$.

Figure 3. Histogram illustrating the method of step height measurement. The histogram shows the distribution of pixel heights, in the area encompassed by the rectangle in Fig. 1c. The two maxima correspond to the upper and lower terraces, respectively. Their separation is the step height.

Figure 4. Frequency of step heights. Steps originating at screw dislocations are not included in this distribution. The total number of observations is 278 . The height $2.5 \mathrm{~A}$ is the average of 23 data points, and the calculated standard deviation is $0.2 \AA$. The height $4.0 \AA$ is

the average of 99 data points, with $0.4 \AA$ standard deviation. The height $6.2 \AA$ is from 142 data points, with $0.3 \AA$ standard deviation.

Figure 5. TEM images of i-Al-Cu-Fe samples after different treatments. (a) Along the $2 \mathrm{f}$ axis, as grown. (b) Along the $2 \mathrm{f}$ axis, after hot isostatic pressing. Arrows point to periodic 
regions in the lattice; one such region is enclosed in a box to show it clearly. The bands of periodic lattice align along the $5 f$ directions. (c) Along the $5 f$ direction, after pressing and annealing to $800^{\circ} \mathrm{C}$. The inset shows a selected area diffraction pattern. The lack of streaking between diffraction spots, and the high degree of perfection in the TEM image, indicate the absence or very low density of "periodic" defect regions. Contrast variations across the images in (a) and (c) are due to variations in foil thickness; in (b), the contrast variation is due to this and also to the strain fields associated with the periodic regions.

Figure 6. LEED pattern of the surface after annealing at $850 \mathrm{~K}$ for 1 hour. The incident beam energy is $70 \mathrm{eV}$.

Figure 7. LEED IV curves from the $5 \mathrm{f}$ surface of Al-Cu-Fe, taken after preparing the surface by sputtering at 15 minutes and annealing at $800 \mathrm{~K}$ for 1 hour, then cooling to $120 \mathrm{~K}$ for data acquisition. Diffraction spot intensity is plotted as a function of momentum transfer parallel to the surface, $\mathrm{Dk}_{\| \mid}$. Each panel includes four symmetry-equivalent curves. At normal incidence, the curves should be identical. The values of $\mathrm{Dk}_{\|}$, based upon $\mathrm{x}$-ray diffraction data for the bulk, are: bottom two panels-- $1.647 \AA^{-1}$; middle two panels-- $2.665 \AA^{-1}$; top two panels--4.312 $\AA^{-1}$.

Figure 8. LEED IV curves from the $5 \mathrm{f}$ surface of $\mathrm{Al}-\mathrm{Cu}-\mathrm{Fe}$, showing the effect of different annealing temperatures on the IV curves. Diffraction spot intensity is plotted as a function of momentum transfer parallel to the surface, $\mathrm{Dk}_{\|}$. Within each box, the lower and upper curves were measured after annealing for one hour to 800 and $850 \mathrm{~K}$, respectively, then cooling to $120 \mathrm{~K}$ for data acquisition. 
Figure 9. R-factors for different terminations of bulk icosahedral Al-Cu-Fe, depending on the surface height $\mathrm{z}$ of the termination. (For each atomic plane shown as a vertical bar, the surface consists of this plane and all planes with higher $\mathrm{z}$ values; planes with lower $\mathrm{z}$ values are cut away.) The terminations giving the best $\mathrm{R}$-factors are marked by arrows. In the lower part, the atomic planes are shown, at their respective depths $z$, as bars with thickness proportional to the atomic density in each layer. The colors of the bars suggest the chemical composition of each layer (as also used in Fig. 12): red is Al-rich with some $\mathrm{Fe}$; green is a roughly even mix of $\mathrm{Al}$ and $\mathrm{Cu}$ with some $\mathrm{Fe}$; dark blue is a roughly even mix of $\mathrm{Al}$ and $\mathrm{Cu}$; and light blue is pure $\mathrm{Cu}$.

Figure 10. Side view (parallel to the surface, which is at top) showing several atomic planes: the five upper ones form a 5-plane layer, the left part of which is cut away to suggest a step down to a 9-plane layer (the structure at the step edge is completely unknown). The colors identify chemical elements: $\mathrm{Al}$ red, $\mathrm{Cu}$ blue, and $\mathrm{Fe}$ green. The larger spacing between the thicker layers is believed to be the preferred place where a surface forms.

Figure 11. Comparison of the IV curves measured experimentally (solid lines) and generated from the best structural model (dashed lines). The experimental curves were measured after annealing at $800 \mathrm{~K}$ for 1 hour. The values of $\mathrm{Dk}_{\|}$, based upon $\mathrm{x}$-ray diffraction data for the bulk, are, for the bottom two boxes-- $1.647 \AA^{-1}$; middle two boxes-$2.665 \AA^{-1}$; top two boxes--4.312 $\AA^{-1}$.

Figure 12. Most favored termination for the five-fold $\mathrm{Al}-\mathrm{Cu}-\mathrm{Fe}$ surface, from LEED. 
Individual atomic planes are shown as colored bars (color coded to suggest their approximate chemical composition). The optimized and bulk interplanar spacings are shown (at right), as well as a possible step height (at left). 

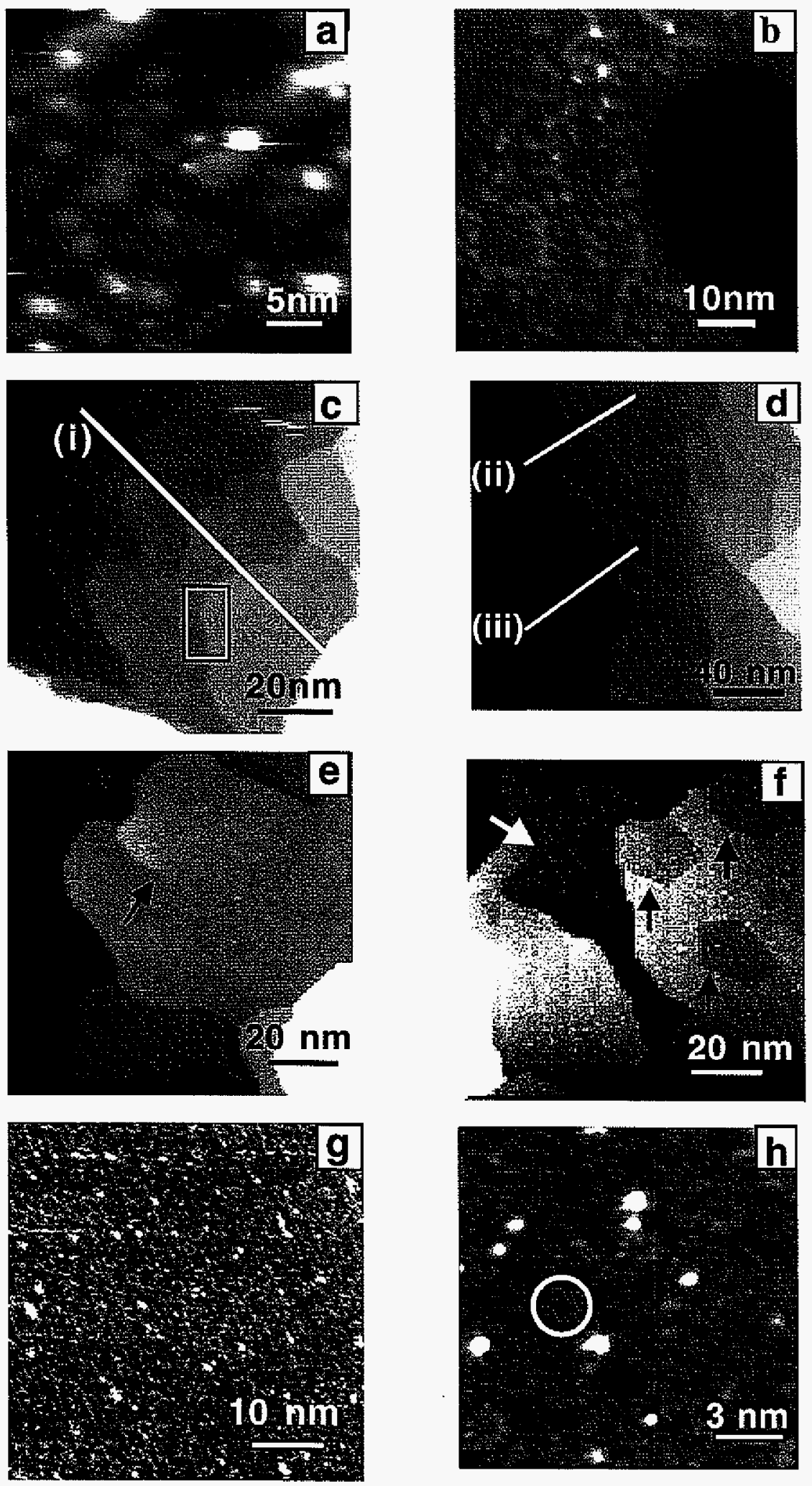

Figure 1 


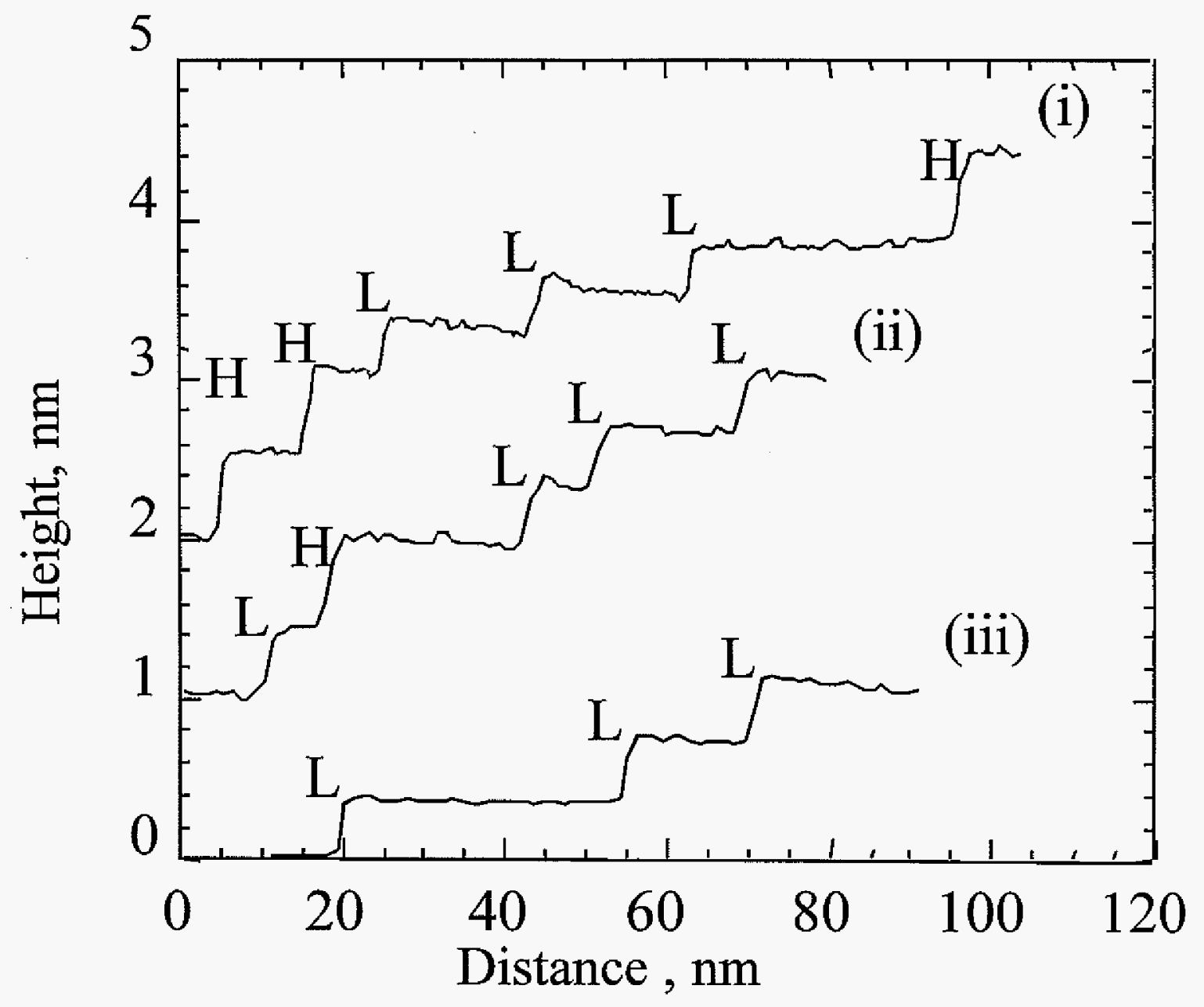

Figure 2 


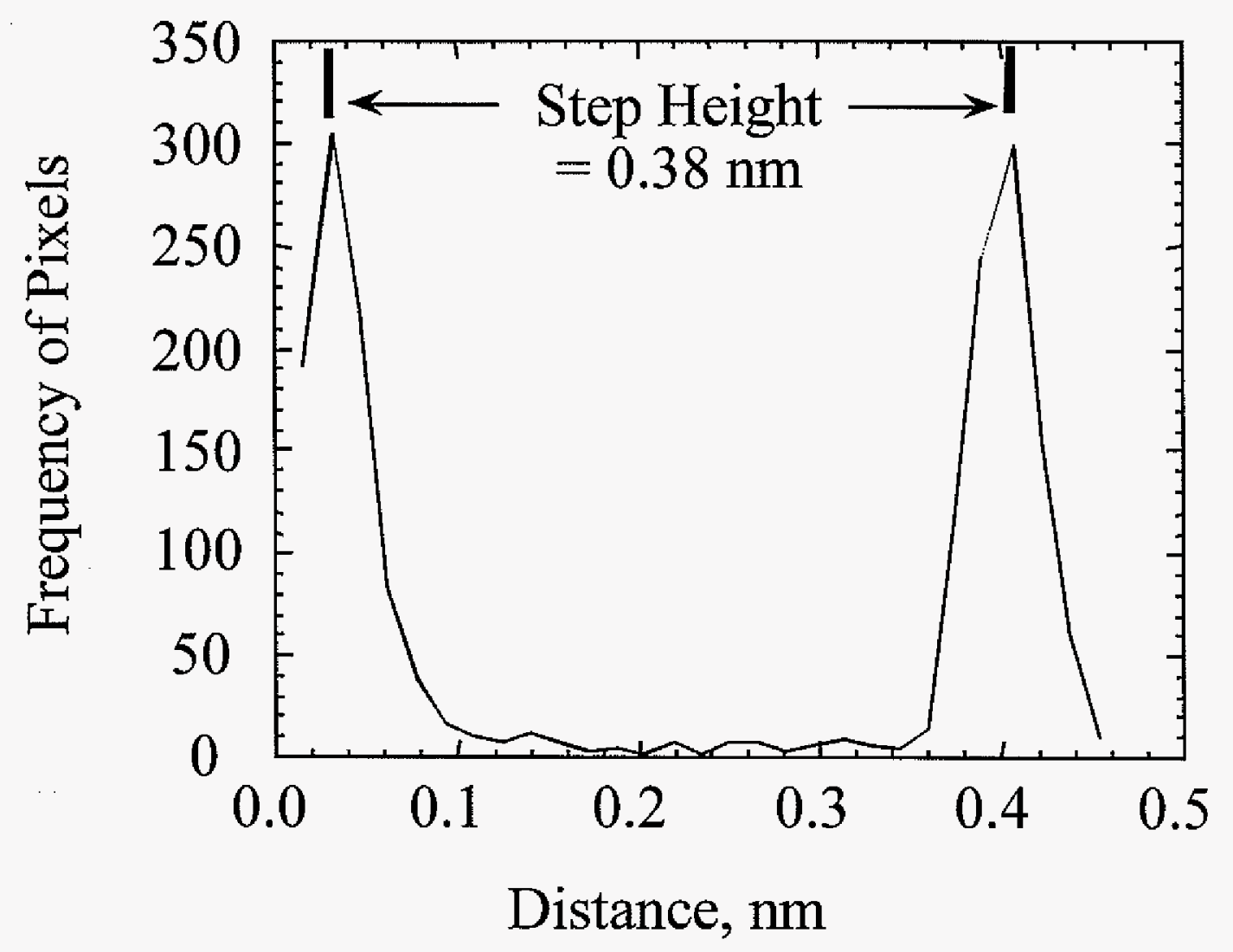

Figure 3 


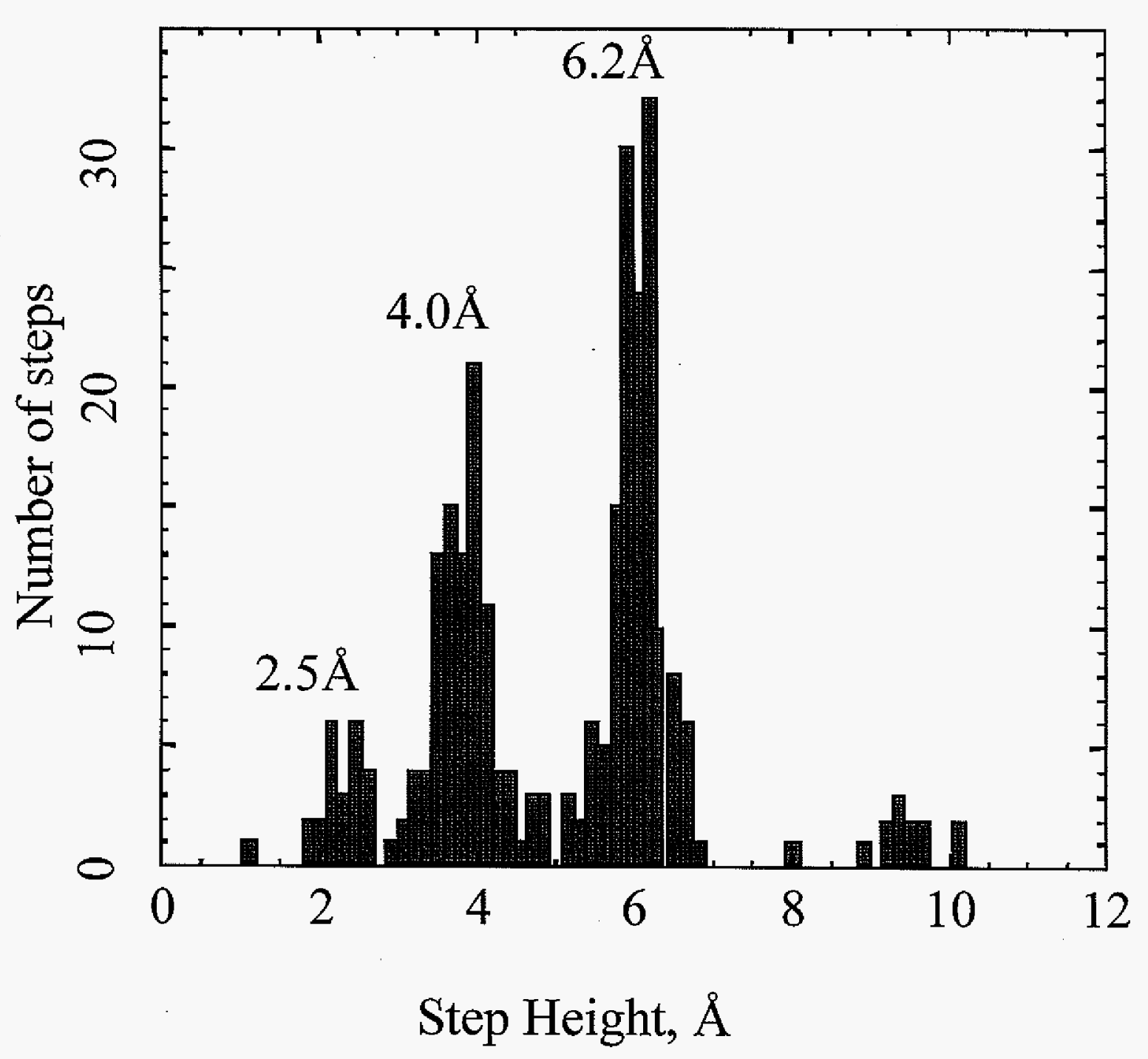

Figure 4 

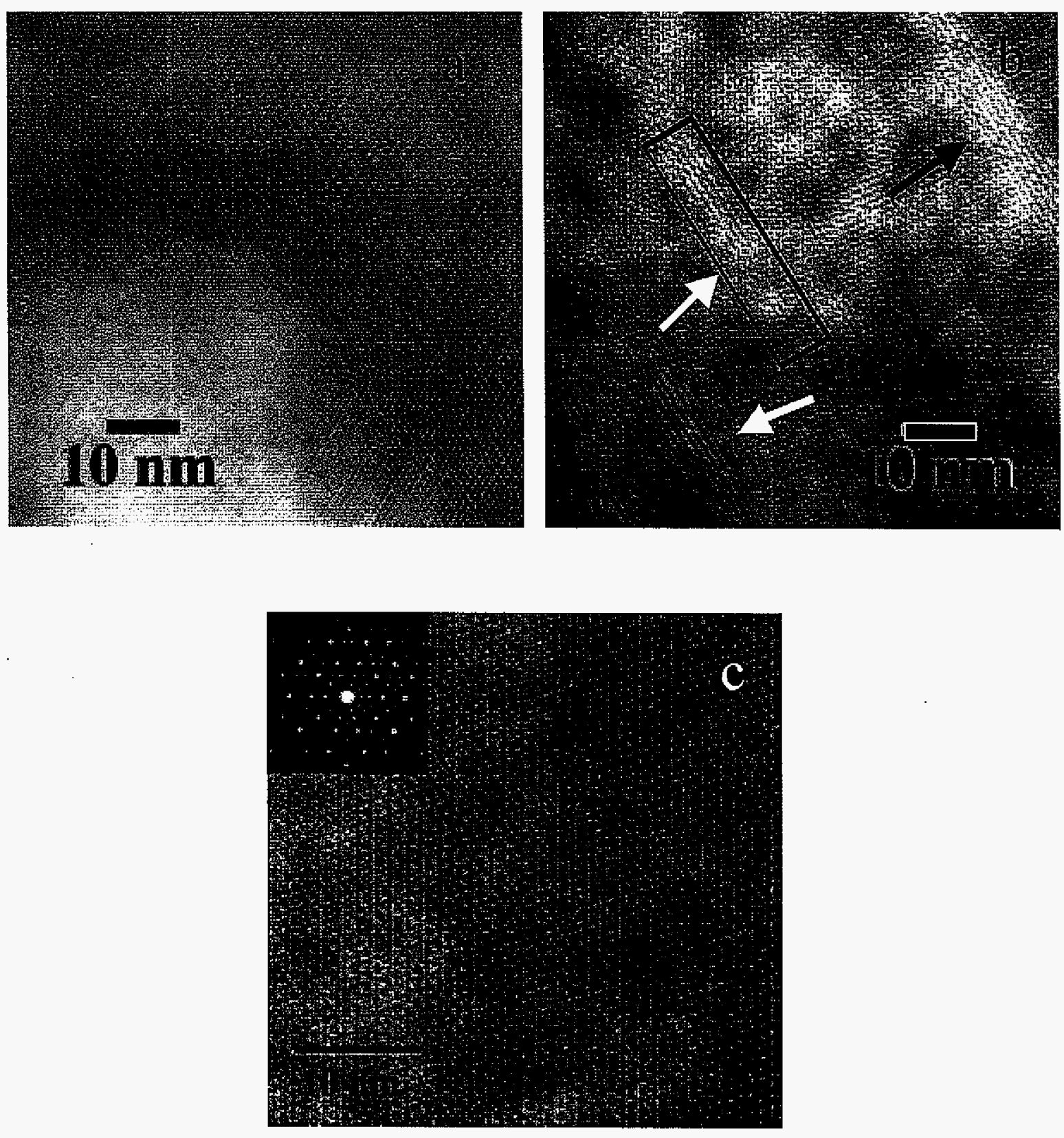

Figure 5 


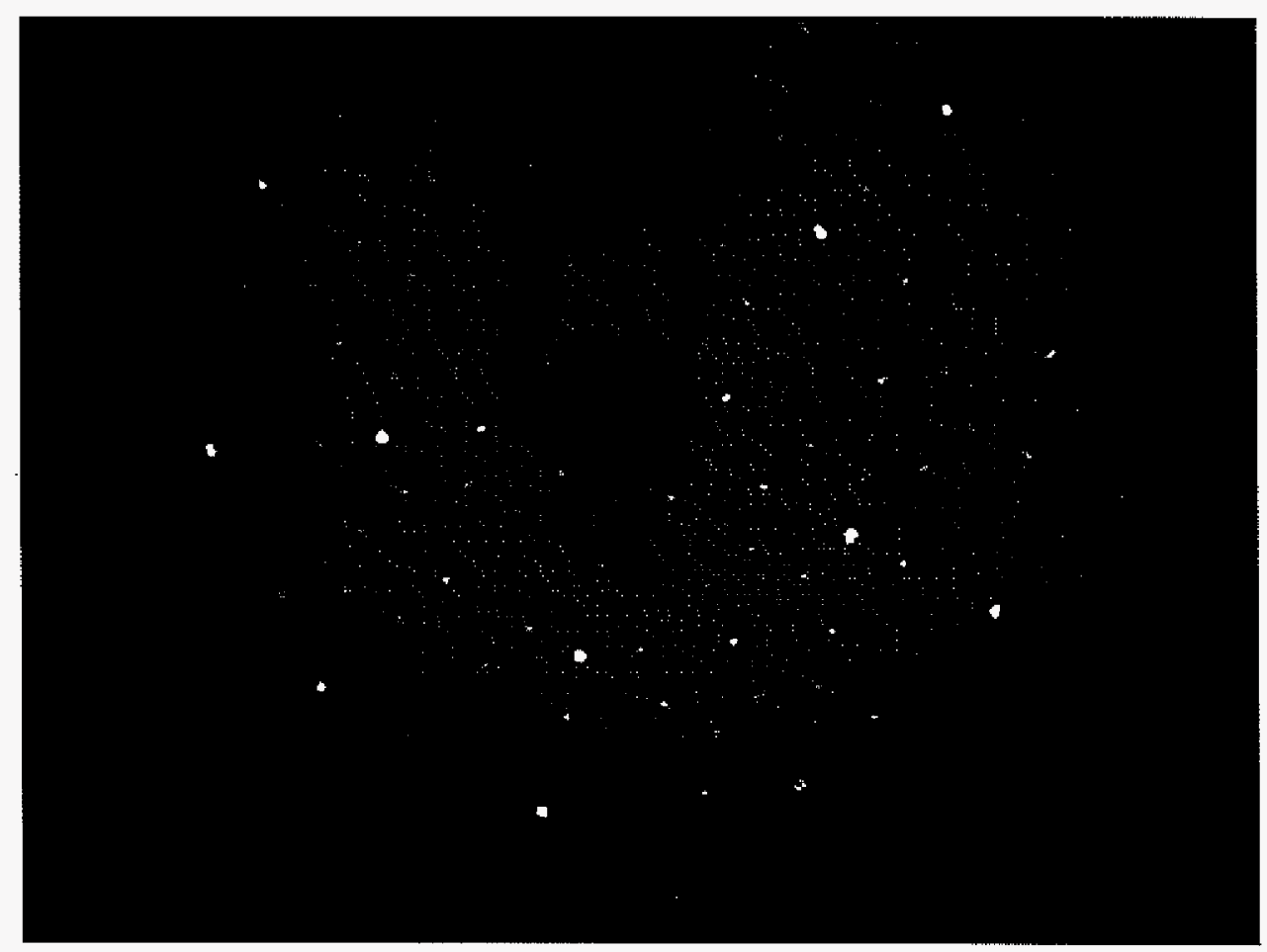

Figure 6 


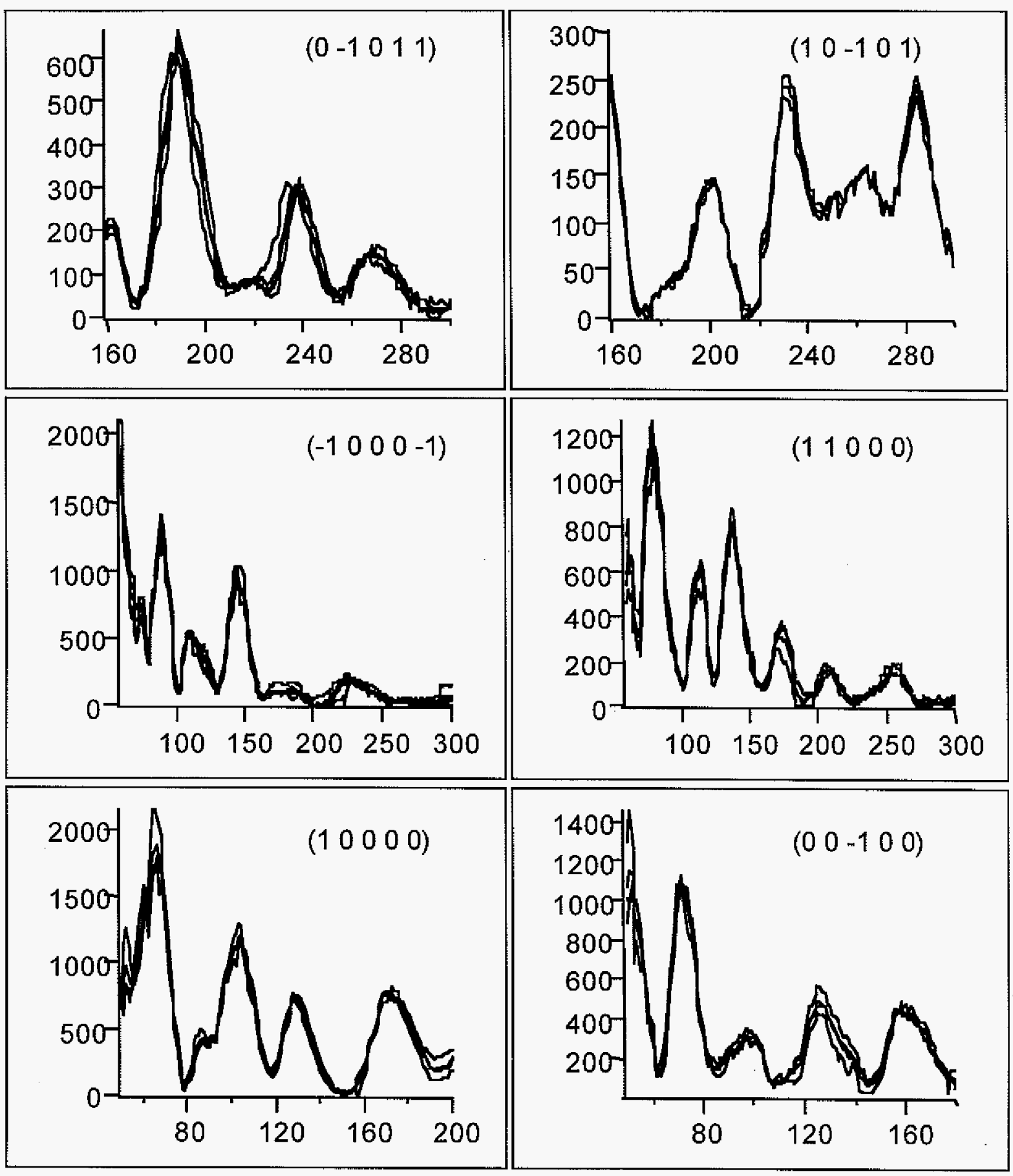

Electron Energy, eV

Figure 7 

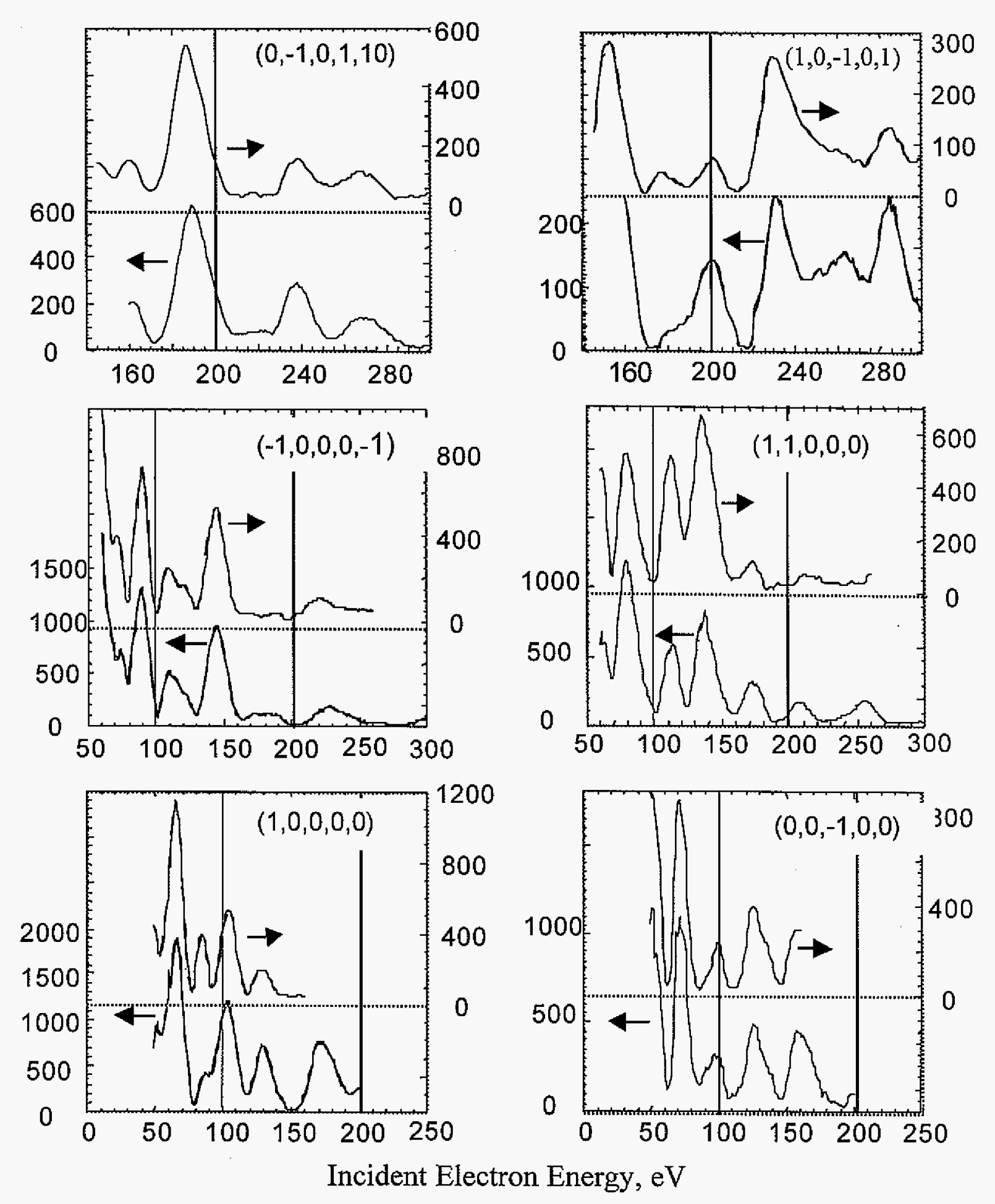

Figure 8 


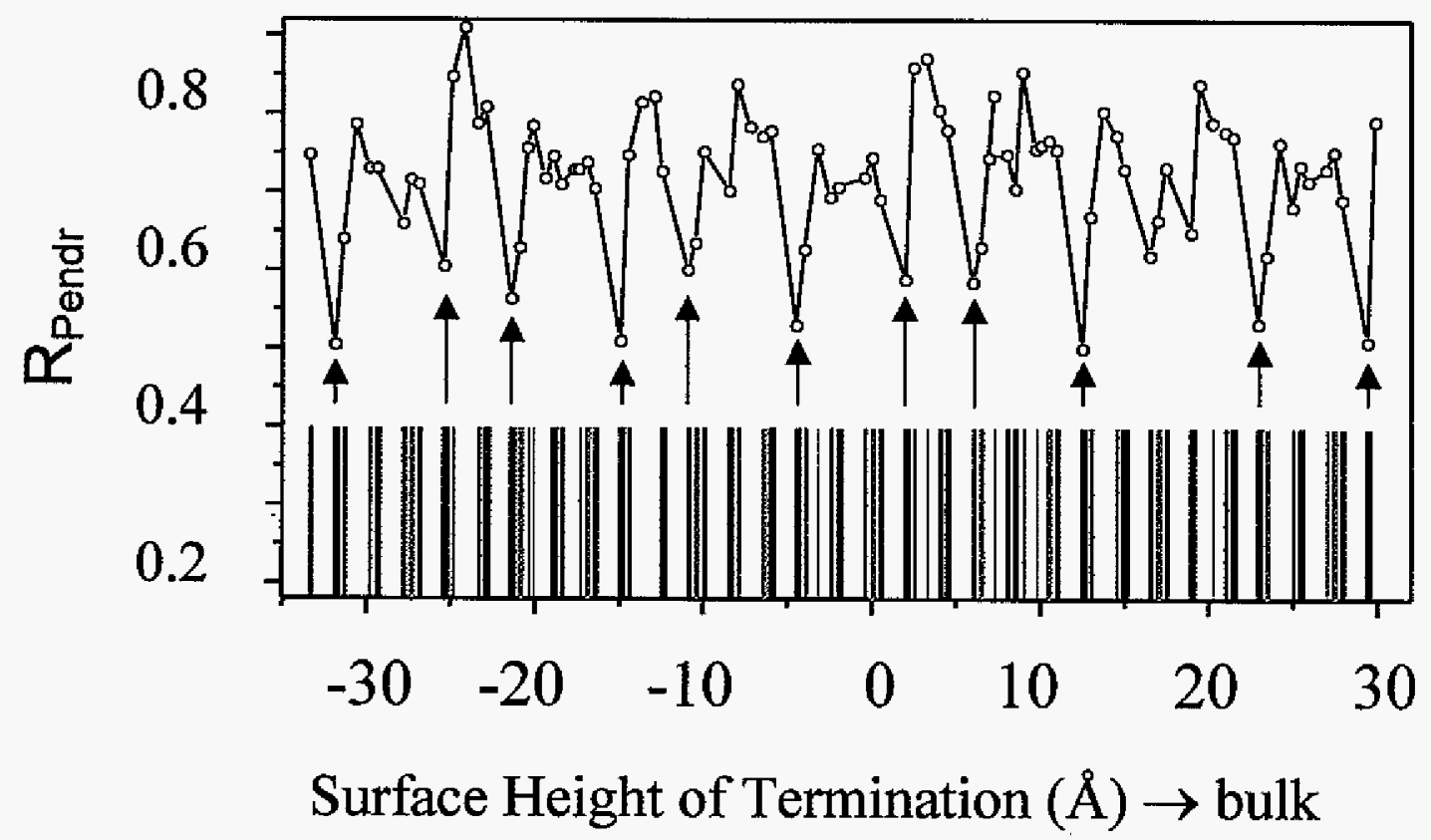

Figure 9 

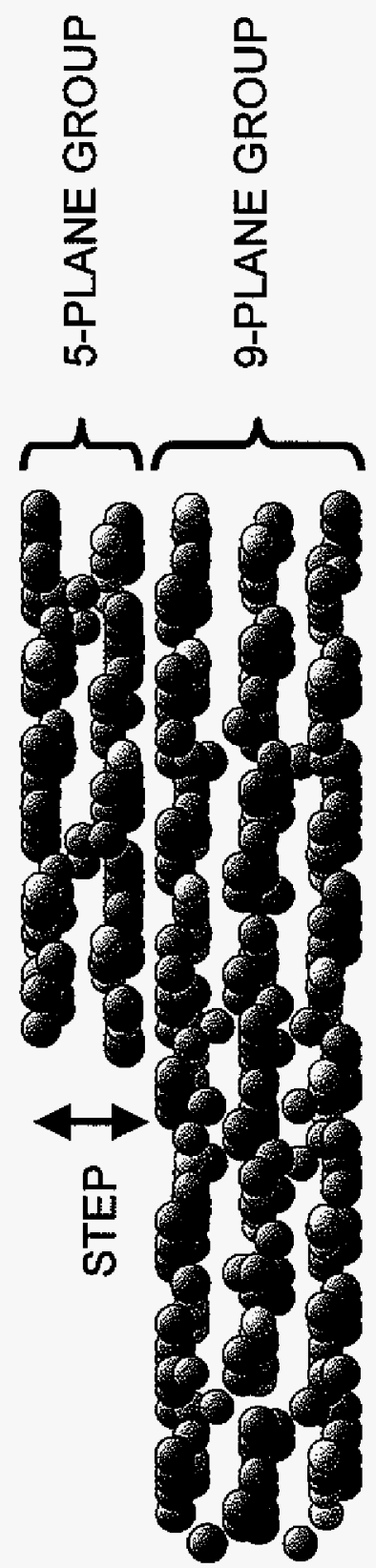

Figure 10 


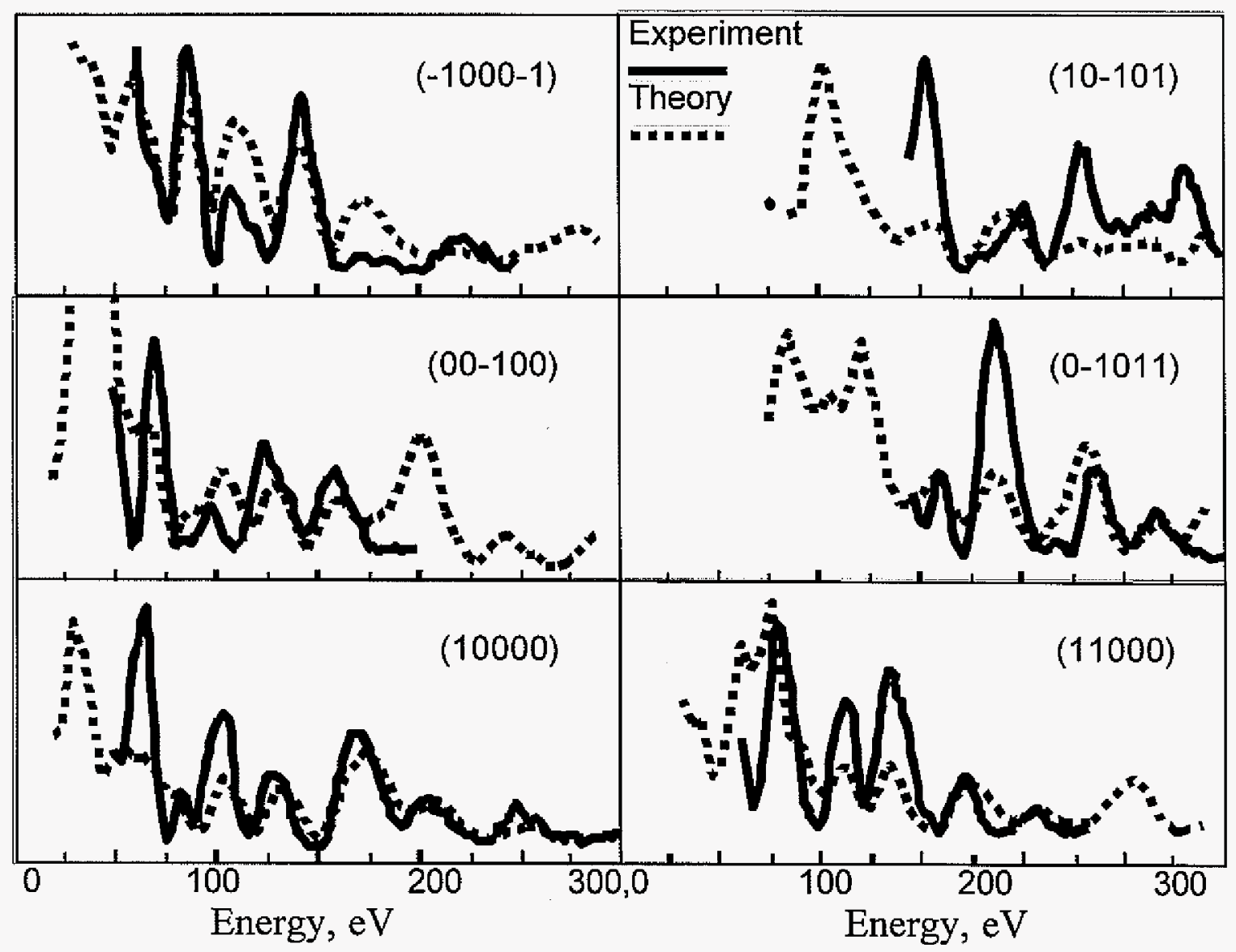

Figure 11 


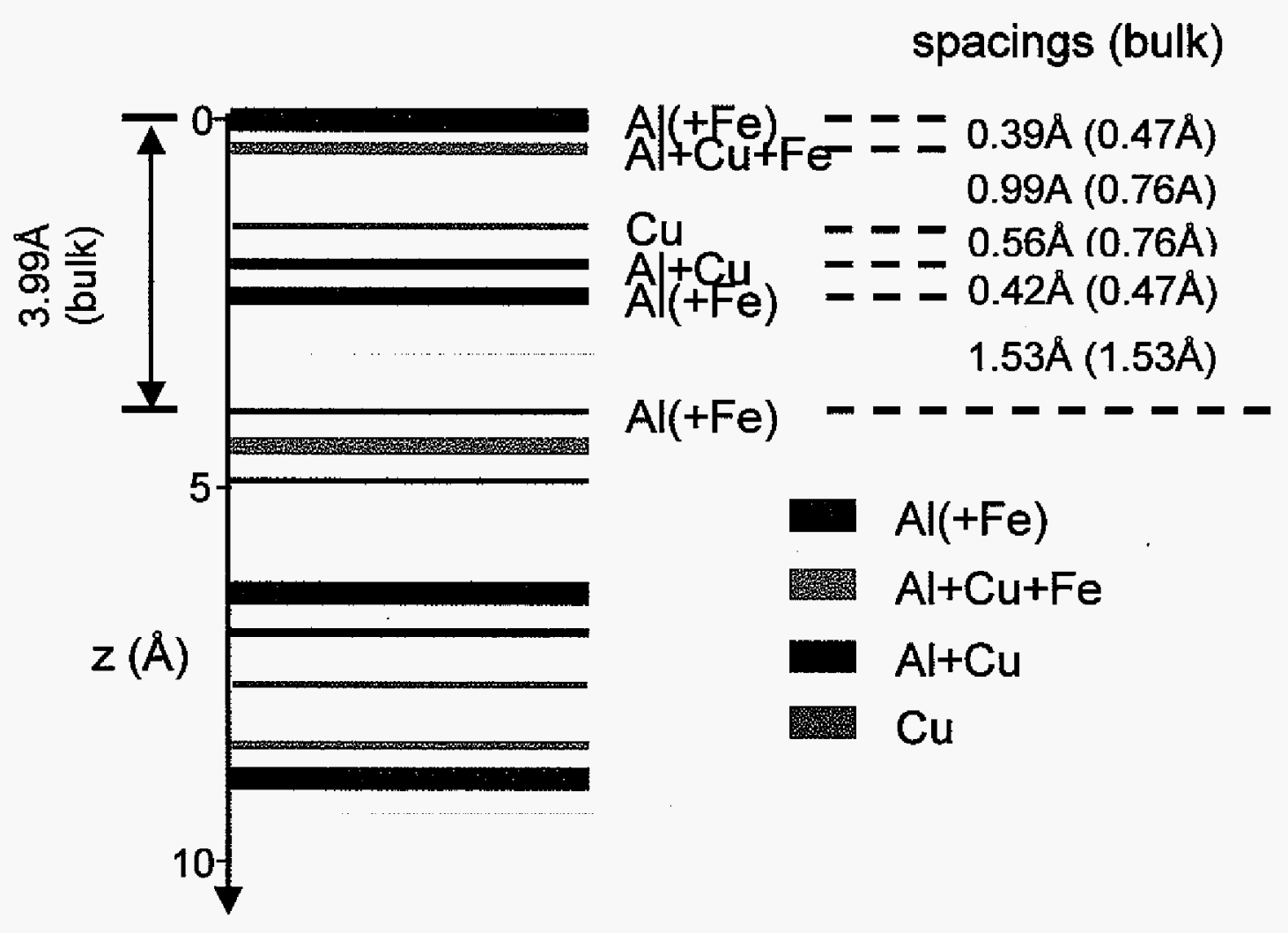

Figure 12 


\title{
AN STM STUDY OF THE ATOMIC STRUCTURE OF THE ICOSAHEDRAL AL-CU-FE FIVEFOLD SURFACE
}

\author{
A Paper submitted to Physical Review B, Rapid Communication \\ T. Cai, V. Fournée, T. Lograsso, A. Ross, and P. A. Thiel
}

PACS Numbers: 61.44.Br $68.35 . \mathrm{Bs} \quad$ 68.37.Ef

\begin{abstract}
We use scanning tunnelling microscopy (STM) to investigate the atomic structure of the icosahedral (i-) Al-Cu-Fe fivefold surface in ultra high vacuum (UHV). Studies show that large, atomically-flat terraces feature many ten-petal "flowers" with internal structure. The observed flower-patterns can be associated with features on $\mathrm{Al}$ rich dense atomic planes generated from two-dimensional cuts of bulk models based on X-ray and neutron diffraction experiments. The results confirm that the fivefold surface of $\mathrm{i}-\mathrm{Al}-\mathrm{Cu}-\mathrm{Fe}$ corresponds to a bulk-terminated plane.
\end{abstract}

The lack of periodicity within the class of intermetallics known as quasicrystals ${ }^{1}$ poses a challenge in determining their surface structure. The most common technique, dynamical theory of electron diffraction, relies very much on the existence of model systems based on periodic structure. Therefore, an exact analysis is not achievable for quasicrystals. Approximations have been introduced successfully into dynamical low energy electron diffraction (LEED) calculations to determine structures of i-Al-Pd-Mn ${ }^{2,3}$ and i-Al-Cu-Fe 
fivefold (5f) surfaces - and the result has been supported in the former case by data from Xray scattering ${ }^{5}$, low-energy ion scattering ${ }^{6}$ and temperature-programmed desorption ${ }^{7}$. However, these techniques provide only averaged information on surface structure. Alternatively, scanning tunnelling microscopy (STM) probes the electronic surface structure at atomic scale in real space ${ }^{8}$ and thus its application does not require periodicity in the material. In this work, we employ STM as our main technique to probe the surface structure of i-Al-Cu-Fe quasicrystal.

STM has been actively used to investigate the structure of the i-Al-Pd-Mn $5 \mathrm{f}$ surface. ${ }^{9-17}$ Annealing the sputtered surface at appropriate temperature routinely generates a step-terrace surface structure. In STM studies, an ultimate goal is to obtain high-resolution images displaying the fine structure (at the best, the direct images of the atomic arrangements) on these flat terraces, and to relate the surface structure to the atomic structure of a bulk model. Two facts challenge the achievement of this goal. First, the intrinsic structural and chemical complexity in the quasicrystals undoubtedly engenders a complex geometric and/or electronic surface structure. Secondly, both the tip specification (the size and the shape) and the scanning conditions greatly influence the resolution and appearance of the STM images. In recent years, several research groups have recorded STM images, with different resolution and at different scales, on flat terraces of i-Al-Pd-Mn $5 f$ surfaces. ${ }^{11-14}$

Among icosahedral quasicrystals, it is known that $\mathrm{i}-\mathrm{Al}-\mathrm{Cu}-\mathrm{Fe}$ has a bulk structure very similar to that of i-Al-Pd-Mn. It is reasonable to expect that surfaces of these two materials with the same high-order symmetry will exhibit similar structure. In this paper, we test that hypothesis. There has been only one previous published STM image of an i-Al-Cu- 
Fe surface, ${ }^{18}$ and it was not possible at that time to attempt interpretation of the observed features, partly due to the lack of information about the bulk structure and partly due to the lack of high-resolution in the imaging.

Fig. 1 represents the step-terrace structure commonly obtained on a clean, well annealed $\mathrm{i}-\mathrm{Al}-\mathrm{Cu}-\mathrm{Fe} 5 \mathrm{f}$ surface in UHV, after preparation as described in other publications. ${ }^{4,19,20}$ We have discussed the step height measurement and the structural imperfection along the $5 \mathrm{f}$ axis in detail in a previous paper. ${ }^{4}$ The step that separates the two major adjacent terraces in Fig. 1 is $0.40 \mathrm{~nm}$ high. The peak-to-peak corrugation across individual terraces is about $0.05 \mathrm{~nm}$. Higher protrusions, about $0.1 \mathrm{~nm}$ high (which appear as the brighter bumps on the terrace), are often observed on the surface as well. These may be similar in origin to the larger protrusions that were reported previously in highly-resolved STM images of the i-Al-Pd-Mn 5 f surface. There, analysis of their shapes led to the conclusion that they should be regarded as part of the icosahedral structure, and not impurities randomly distributed on the surface. ${ }^{16}$

High-resolution STM (Fig. 2a) shows the fine structure on the flat terraces. The Fourier Transform from such a image of $50 \times 50 \mathrm{~nm}^{2}$ (see inset in Fig. 2a) exhibits tenfold symmetry, consistent with the $5 \mathrm{f}$ quasiperiodic order on the terrace. The most notable features in the image are the "flowers" that are approximately $1.5 \mathrm{~nm}$ in diameter, measured from peak to peak of the outermost petals, as shown in Fig. 2c. Several flowers are outlined in Fig. 2a, and labelled A-C. In their pioneering STM work on i-AI-Cu-Fe $5 f$ surface, Becker et al. ${ }^{18}$ also reported some "daisy"-like structures that appeared like central spots surrounded by a series of ring- or U-shaped features. However, features in their STM image were not sufficiently resolved. We have obtained STM images at higher resolution, which makes it 
possible to analyse the structural features of the flowers. The "complete" flowers (Fig. 2a, A-C) consist of ten petals. Despite the distortion of the STM scan, the ten petals appear to be uniformly distributed (as far as both intensities and positions are concerned, e.g. flower A) in a circle, which gives local tenfold symmetry. However, some flowers are missing parts of petals (e.g. flower B). The center of the flowers is either filled (e.g. flowers A and B) or empty (e.g. flower C). Between the center and the ring of ten petals in each flower, there is clearly another ring of bright contrast, which, however, is not well resolved in the STM images. These features are clarified in the schematic sketches of Fig. 2c.

It is clear that there is also a wealth of features between the intact flowers, which we call the 'glue' area. In some cases, four close petals from two neighbouring flowers (two petals from each flower) constitute four vertices of a pentagon, with an additional atom that does not belong to any flowers fulfilling the fifth vertex. Such pentagons appear much more clearly in Fig. 2b. Another type of glue object is a nearly petal-free inner ring, with a diameter of $1.05 \mathrm{~nm}$, shown in Fig. 2a. Such a ring can be resolved into ten separate spots, defining axes parallel to the axes of the petal-rich flowers, suggesting that the inner rings have this symmetry in the full flowers as well. Overlapping is another possible configuration for adjacent flowers, as indicated by the white arrows in Fig. 2a.

Some groups of five flowers form large pentagons, as outlined in Fig. 3. Noting that the lines linking the center and one of the ten petals of each flower define five directions, we connect the flowers by five sets of parallel lines in the five directions defined above. Fig. 3 shows only part of one set of parallel lines to illustrate the idea. Analysis shows that these lines are mainly separated by two different spacings: $\mathrm{L}=1.88 \mathrm{~nm}$ for the wider spacings and 
$\mathrm{S}=1.17 \mathrm{~nm}$ for the narrower ones. The ratio $\mathrm{L} / \mathrm{S}=1.61$ is close to the "golden mean" $\tau=$ $(\sqrt{5}+1) / 2 \approx 1.618$.

The arrow in Fig. 3 indicates a location where one parallel line stops and shifts at right angles. Such discontinuity and displacement changes the sequence of the consecutive spacings, e.g., from a LLS sequence to a LSL one, which makes it impossible to identify the exact sequence of successive spacings. Another reason why it is difficult to identify exact sequences is because one could also identify other, more closely-spaced lines connecting flower centers, particularly when dealing with extended images of larger area. An example is shown by the upper-right-most spacing in Fig. 3. The wider spacing (L) then would be reasonably split into two shorter spacings of $S$ and $S / \tau$ (note $L=S+S / \tau$ ).

Based on X-ray and neutron diffraction measurements, Boudard et al. ${ }^{21}$ derived a bulk model for i-Al-Pd-Mn. The bulk structure can be described as atomically-dense planes, quasi-periodically stacked along the $5 \mathrm{f}$ axis, and separated by less-dense planes. The atomically-dense planes can be separated into a small number of groups, each group encompassing only a small range of variations in composition and local order.

Similar experiments have been carried out on the i-Al-Cu-Fe alloy, ${ }^{22}$ and a similar structure model can be used to describe its bulk structure. Based on this model, dynamic LEED calculations ${ }^{4}$ concluded that the outermost layer perpendicular to $5 \mathrm{f}$ axis is Al-rich with a fraction of Fe atoms. Fig. 4 presents a $10 \times 10 \mathrm{~nm}^{2}$ section of such a plane, generated from the bulk model. This atomically-dense plane contains $85 \% \mathrm{Al}$ atoms and $15.0 \% \mathrm{Fe}$ atoms.

Many flower-like patterns, with size similar to those that dominate the STM images, are easily identified in the plane (Fig. 4). Each flower consists of two concentric rings with a 
filled center. Each ring possesses ten atoms. However, the choice of atoms to form such flower-patterns in the plane is not unique. We have chosen the flowers in Fig. 4 in such a way that their arrangement matches the pattern of flowers drawn in the STM image of Fig. 3, and vice versa. As observed in STM images, local 'glue' configurations that connect the neighbouring flowers, such as the small pentagons, overlapping flowers, and intermediatesized rings, are also identified in the plane, as indicated by the constructions in Fig. 4.

The size of the features identified in the model (Fig. 4) compares very well with the size of the features measured with STM, as indicated in Table 1.

Furthermore, we note that the centre of the patterns identified in Fig. 4 is either occupied by $\mathrm{Al}$ atom or $\mathrm{Fe}$ atom. In addition, some flower-patterns have $\mathrm{Fe}$ atoms, instead of only Al atoms, occupying the atomic positions in the petals. Comparing the obtained STM image (Fig. 3) where the flowers are either filled or empty at center and some flowers exhibit missing petals, we speculate that $\mathrm{Fe}$ and $\mathrm{Al}$ atoms contribute differently to the electronic structure at the surface and thus respond differently to the tunnelling conditions used to acquire the presented STM images. Another possible interpretation for the missing parts (both the empty center and the missing petals) is local defects on the surface.

Finally, we point out that the atomic-resolution STM images of i-Al-Cu-Fe presented in this paper are quite different than those for the i-Al-Pd-Mn $5 \mathrm{f}$ surface. On the latter surface, pentagonal holes are the predominant features reported to date. ${ }^{11-14,17,23}$ We have ruled out differences in experimental conditions in various laboratories as being responsible for the differences, as follows: in the same chamber, we acquired a $30 \times 30 \mathrm{~nm}^{2}$ highresolution STM image on an i-Al-Pd-Mn 5 f surface (Fig. 5) with the same tip under similar tunnelling conditions. The most notable features in Fig. 5 are the pentagonal depressions 
aligned in parallel directions. Nonetheless, images for both alloys can be interpreted in terms

of features expected for the bulk-terminated surfaces. ${ }^{17}$ Hence, it is puzzling why the surface STM images are very different, when the bulk structures of the two quasicrystals are very similar. Perhaps subtle differences in the electronic structure simply serve to emphasize the pentagonal holes in one alloy, and the flowers in the other.

In summary, we have obtained the first atomic resolution STM images on the atomically-flat terraces of $\mathrm{i}-\mathrm{Al}-\mathrm{Cu}-\mathrm{Fe} 5 \mathrm{f}$ surface. The analysis of the images shows that the flat terraces exhibit local $5 f$ symmetry, consistent with the quasiperiodicity on the surface. The acquired STM images display the atomic arrangements on the surface with sufficient resolution so that it is possible to compare the STM image directly with the atomicallydense planes generated from the experimental bulk structure. The comparison shows a good match. We conclude that these images are consistent with the description of the $5 \mathrm{f}$ surface of i-Al-Cu-Fe as Al-rich, bulk-terminated planes.

\section{Acknowledgements}

This work was supported by the Director, Office of Science, Office of Basic Energy Science, Materials Science Division of the U. S. Department of Energy under contract No. W-405-Eng-82. We would also like to acknowledge that STM studies of the atomic-scale structure of 5f Al-Pd-Mn surfaces were underway in parallel with our own work, in the laboratories of R. McGrath (Univ. Liverpool) and D. Gratias (LEM-CNRS/ONERA). We thank them for sharing information openly and generously. 


\section{References}

1. C. Janot, Quasicrystals: A Primer (Clarendon Press, Oxford, 1992).

2. M. Gierer, M. A. Van Hove, A. I. Goldman, et al., Phys. Rev. Lett. 78, 467 (1997).

3. M. Gierer, M. A. Van Hove, A. I. Goldman, et al., Phys. Rev. B 57, 7628 (1998).

4. T. Cai, F. Shi, Z. Shen, et al., Surface Science, in press (2001).

5. M. J. Capitan, J. Alvarez, J. L. Joulaud, et al., Surface Science 423, L251 (1999).

6. R. Bastasz, C. J. Jenks, T. A. Lograsso, et al., in MRS Conference Proceedings:

Quasicrystals, edited by E. Belin-Ferré, P. A. Thiel, K. Urban and A.-P. Tsai (MRS, Warrendale, NJ, 2001).

7. C. J. Jenks, T. A. Lograsso, and P. A. Thiel, J. Am. Chem. Soc. 120, 12668 (1998).

8. C. Bai, Scanning Tunnelling Microscopy and Its Applications, 2000).

9. P. Ebert, M. Feuerbacher, N. Tamura, et al., Phys. Rev. Lett. 77, 3827 (1996).

10. P. Ebert, F. Kluge, B. Grushko, et al., Phys. Rev, B 60, 874 (1999).

11. T. M. Schaub, D. E. Bürgler, H.-J. Güntherodt, et al., Phys. Rev. Lett. 73, 1255 (1994).

12. T. M. Schaub, D. E. Bürgler, H.-J. Güntherodt, et al., Z. Phys. B 96, 93 (1994).

13. T. M. Schaub, D. E. Bürgler, H.-J. Güntherodt, et al., Appl. Phys. A 61, 491 (1995).

14. T. M. Schaub, D. E. Bürgler, C. M. Schmidt, et al., J. Non-Cryst. Solids 205-207, 748 (1996).

15. Z. Shen, C. Stoldt, C. Jenks, et al., Phys. Rev. B 60, 14688 (1999).

16. J. Ledieu, A. Munz, T. Parker, et al., Surface Science 433-435, 666 (1999).

17. J. Ledieu, R. McGrath, R. D. Diehl, et al., Surface Sci. Lett. 492, L729 (2001).

18. R. S. Becker, A. R. Kortan, F. A. Thiel, et al., J. Vac. Sci. Technol. B 9, 867 (1991). 
19. C. J. Jenks, P. J. Pinhero, Z. Shen, et al., in Proceedings of the 6th International Conference on Quasicrystals (ICQ6), edited by S. Takeuchi and T. Fujiwara (World Scientific, Singapore, 1998), p. 741.

20. Z. Shen, P. J. Pinhero, T. A. Lograsso, et al., Surface Sci. 385, L923 (1997).

21. M. Boudard, M. de Boissieu, C. Janot, et al., J. Phys. Condens. Matter 4, 10149 (1992).

22. M. Cornier-Quiquandon, A. Quivy, S. Lefèbvre, et al., Phys. Rev. B 44, 2071 (1991).

23. T. Schaub, J. Delahaye, C. Berger, et al., European Physical Journal B 20, 2001 (2001). 


\section{Table and Figure Captions}

Table 1. Experimental and model-based sizes of characteristic features.

Figure 1. STM image $\left(100 \times 100 \mathrm{~nm}^{2}\right)$ showing the typical step-terrace structure. The arrow indicates a screw dislocation; these are often observed on the $5 \mathrm{f}$ i-Al-Cu-Fe surface. ${ }^{4}$

Figure 2. a) High resolution STM image $\left(20 \times 20 \mathrm{~nm}^{2}\right)$ of an atomically-flat terrace.

Inset: Fourier Transform of an image of $50 \times 50 \mathrm{~nm}^{2}$ on a terrace. A: complete flower with ten petals and filled center. B: flower with one missing petal and filled center. C: flower with empty center. D: nearly petal-free flower. Arrows: overlapping of neighbouring flowers.

b) High resolution STM image $\left(10 \times 20 \mathrm{~nm}^{2}\right)$ showing small pentagons gluing two adjacent flowers. Large circles: flower patterns. Small circle: outlines of small gluing pentagons.

c) Schematic of a flower, together with line scans of a flower with filled center (top curve in box), and an empty center (lower curve in box).

Figure 3. Same high resolution STM image as in Fig. 2. Pentagons are drawn to illustrate the local $5 \mathrm{f}$ arrangements of flowers. Parallel lines separated by different spacings align the flowers. Arrow indicates where a displacement takes place. The splitting of a wider spacing into two smaller ones is shown in the upper-right-most spacing.

Figure 4. Atomic positions in a plane $\left(10 \times 10 \mathrm{~nm}^{2}\right)$ generated from experimental bulk structure model. Filled circles: Al atoms. Unfilled circles: Fe atoms. The sketched contour illustrates the matching part of STM image outlined in Fig. 3.

Figure 5. High resolution STM image $\left(20 \times 20 \mathrm{~nm}^{2}\right)$ of an i-Al-Pd-Mn $5 f$ surface, obtained under experimental conditions very similar to those of Fig. 2-3, and with the same experimental apparatus. 
Table 1

\begin{tabular}{|c|c|c|}
\hline Feature & $\begin{array}{c}\text { Size in } \\
\text { STM data (nm) }\end{array}$ & $\begin{array}{c}\text { Size in model } \\
\text { (nm) }\end{array}$ \\
\hline Full flowers (petal-to-petal) & 1.57 & 1.52 \\
\hline Inner ring & 1.05 & 0.94 \\
\hline Small pentagon (edge length) & 0.46 & 0.47 \\
\hline Large pentagon (edge length) & 2.04 & 1.99 \\
\hline L (defined in Fig. 3) & 1.88 & 1.89 \\
\hline S (defined in Fig. 3) & 1.17 & 1.17 \\
\hline L/S & 1.61 & 1.61 \\
\hline
\end{tabular}




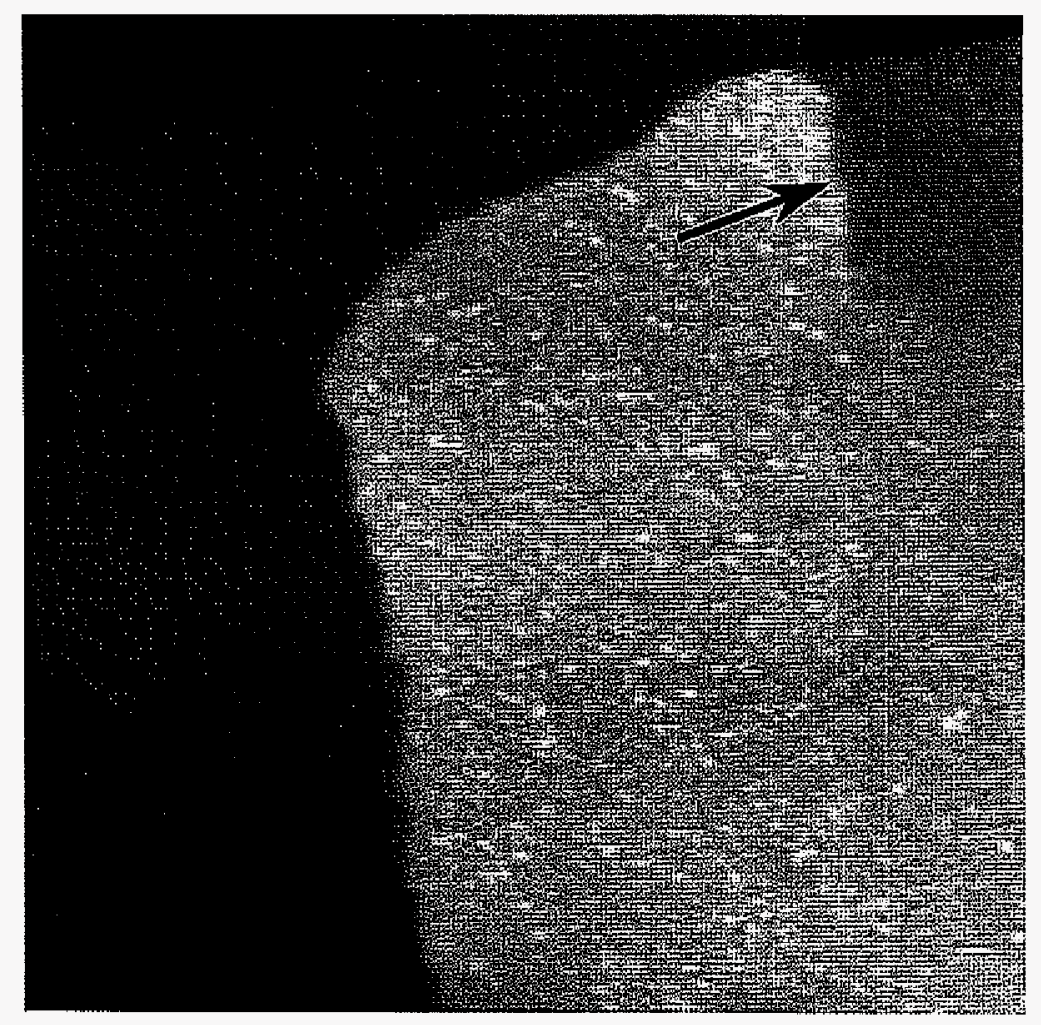

Figure 1 

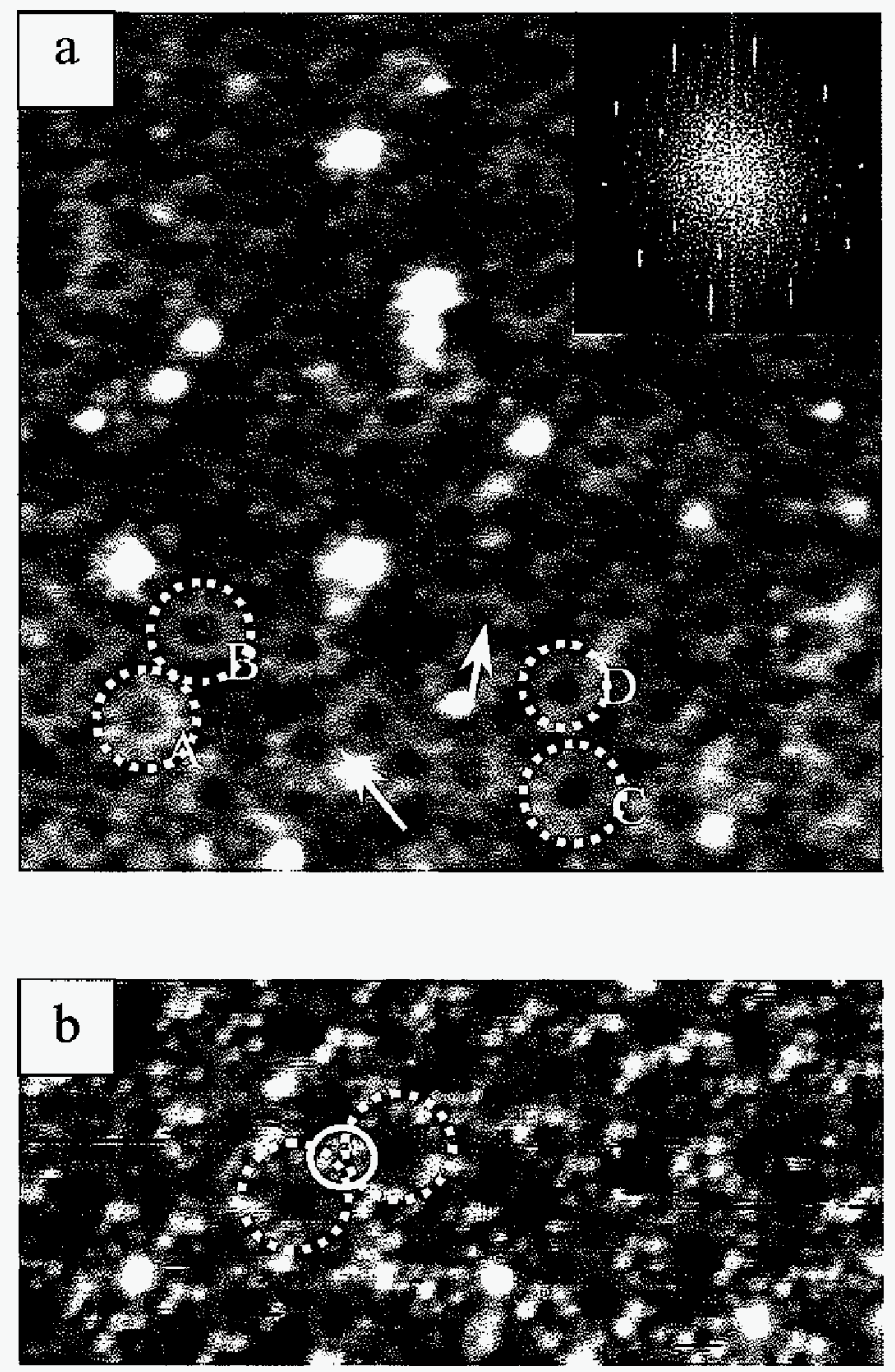

Figure 2 


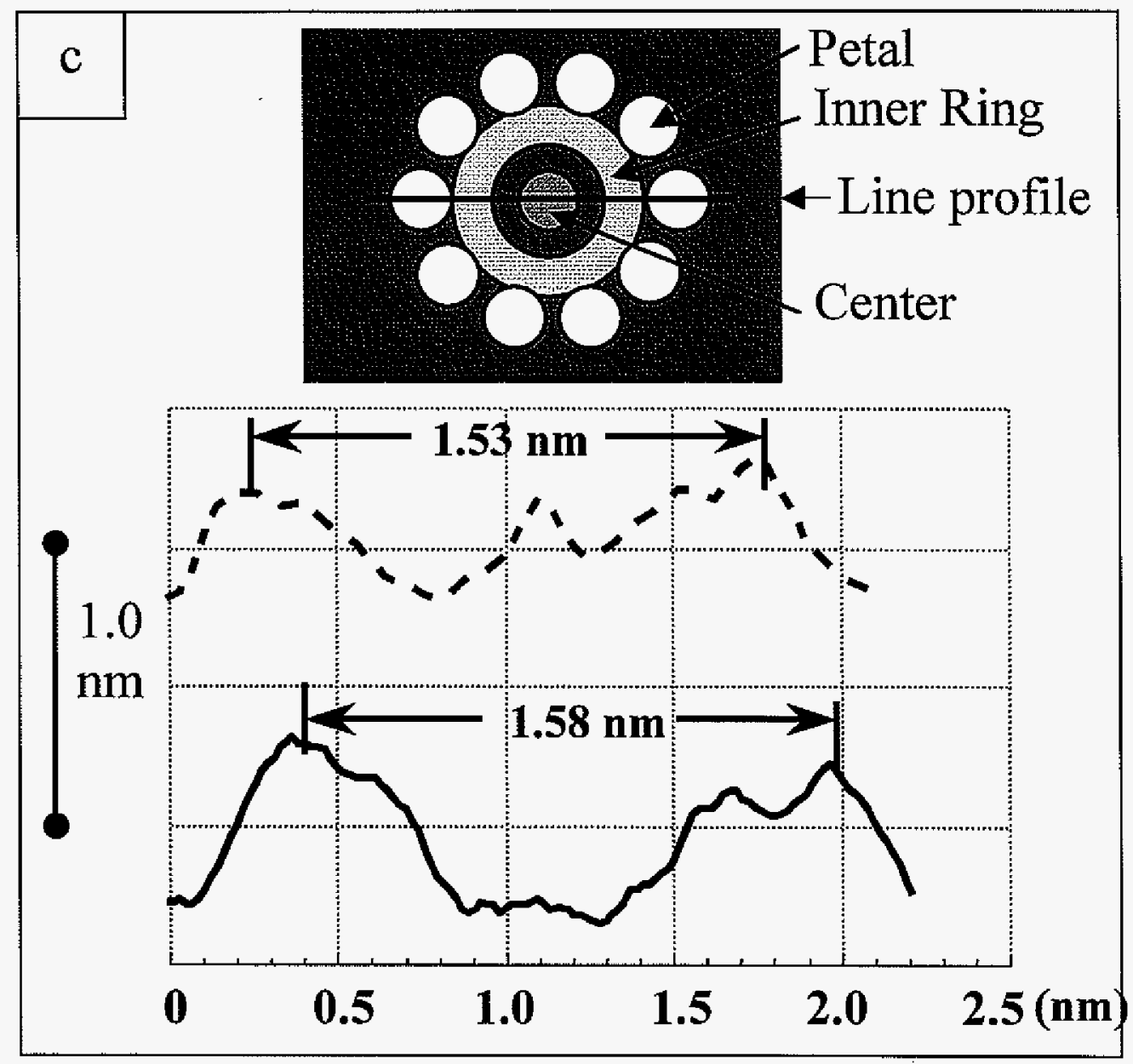

Figure 2 


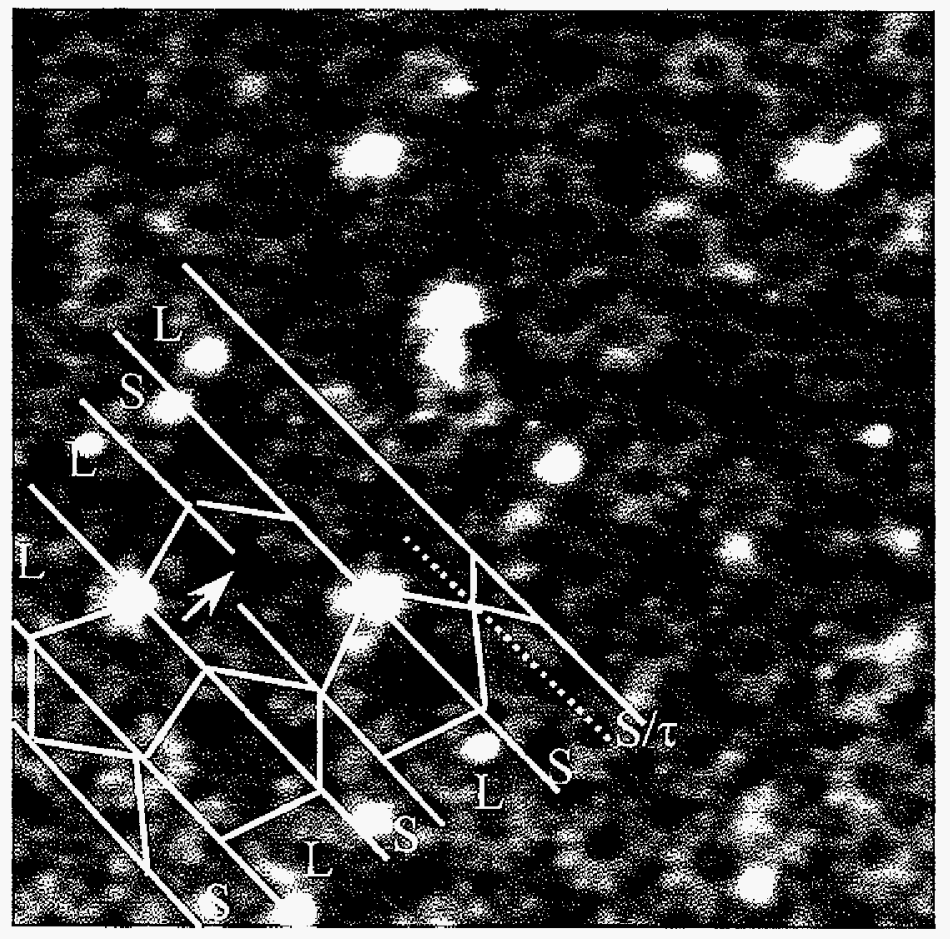

Figure 3 




Figure 4 


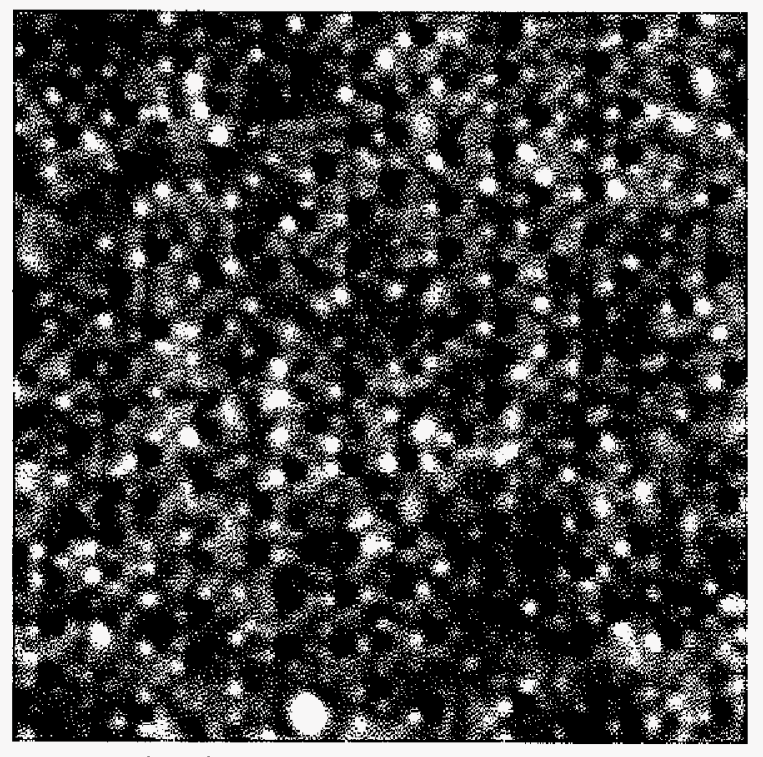

Figure 5 


\section{PSEUDOMORPHIC STARFISH: ARRANGEMENT OF EXTRINSIC METAL ATOMS ON A QUASICRYSTALLINE SUBSTRATE}

A paper submitted to Nature

T. Cai", J. Ledieu*, V. Fournée, T. Lograsso, A. Ross, R. McGrath, P. Thiel

Quasicrystals $^{1}$ - intermetallics with long-range order but without periodicity present a number of unsolved mysteries, among them the question of how they form. This is because the "rules" that govern their order can only be grasped, either by using geometricalmathematical constructs such as the Fibonacci sequence and the Penrose tiling, or by resorting to high-dimensional space. In either case, it is difficult to envision how nature actually implements these non-intuitive rules, placing one atom after another to form a real three-dimensional structure with excellent long-range order. One direct approach to this intellectual conundrum is to observe the way that extrinsic metal atoms arrange themselves on a quasicrystalline template (surface), at the atomic scale. Here we show how $\mathrm{Al}$ atoms deposited on an Al-rich quasicrystal nucleate and grow into islands. Nucleation occurs at specific surface sites, and growth preserves the fivefold symmetry of the substrate over a short range.

In this paper, we report atomic-scale observations of, and a model for, the initial nucleation of small, pseudomorphic features on a quasicrystal surface. We choose the clean fivefold surface of icosahedral $\mathrm{Al}-\mathrm{Cu}-\mathrm{Fe}$ as the substrate. We choose $\mathrm{Al}$ as the deposit, because this matches the chemical composition of most of the exposed surface layer. ${ }^{2}$

\footnotetext{
*These authors contributed equally to the work.
} 
Hence, chemically, this system is very near to simple homoepitaxial growth. Structurally, things are more complex, since it is well known that the structure of bulk $\mathrm{Al}$ is face-centered cubic; however, the film's interaction with the substrate might lead it to deviate from this arrangement and adopt fivefold symmetry (pseudomorphic growth).

Figure 1 illustrates a typical scanning tunnelling micrograph obtained after deposition of a low coverage (in this case, 0.04 monolayers) of $\mathrm{Al}$ from a thermal filament in ultrahigh vacuum. Most of the image encompasses a single terrace of the quasicrystalline surface. The substrate is the red region, part of which is shown in the rectangle, at a contrast that reveals the fine structure of the nascent surface. Within this fine structure, many flowerlike features can be identified. An abundance of evidence, ${ }^{2-4}$ including analysis of the flowers and their relative positions, ${ }^{5}$ suggests that quasicrystalline surfaces of this type are laterally bulk-terminated, i.e. the atoms in the surface plane occupy the same relative positions as if they were buried in the bulk. (There is, however, a deviation in the interplanar spacing near the surface. ${ }^{2,6-8}$ )

The contrast is chosen in the remainder of the image so as to highlight the features formed by the deposited $\mathrm{Al}$ atoms at room temperature. These features resemble starfish. All are aligned in parallel, even across different terraces. All of the starfish are the same size and shape, although a few lack one or more "legs." The distance between the highest points on adjacent legs defines a pentagon with sides of $5.1 \pm 0.2 \AA$. Formation of the starfish is very robust, being insensitive to deposition flux over a range of two orders of magnitude $\left(8 \times 10^{-5}\right.$ to $7 \times 10^{-3}$ monolayers/s). These observations are highly reproducible.

A first task is to identify the sites occupied by the starfish, relative to the bulkterminated surface structure. Three pieces of information are useful in this task: the fact that 
all starfish are aligned in parallel; the size of the starfish; and the orientation of the starfish relative to the substrate flowers. Using Boudard's model ${ }^{9}$ for the bulk structure, and assuming that the substrate is bulk-terminated, the fact that all the starfish are aligned in parallel allows several types of adsorption site to be eliminated. For instance, the centres of the decagonal flowers should give two equally probable directions for the pentagonal $\mathrm{Al}$ stars (rotated to $\pi / 5$ with respect to each other) whereas only one is seen. By further considering the orientation of the starfish with respect to the substrate features from plane to plane, three good candidates emerge: empty (light gray), partially filled (hatched gray) and small (black) pentagons (see Fig.2). However, the last two pentagons suffer from the same problem as the centers of the flowers: they point in two equally-probable directions. Moreover, the atomic distribution within the hatched pentagons is asymmetric, which should lead to distorted starfish. This is clearly not observed. Due to the short edge length of the black pentagons $(2.901 \AA)$ they seem unlikely as sites for starfish formation. Hence, these two types of pentagons are ruled out, leaving the empty pentagons as the best candidates for the starfish sites.

Furthermore, the centres of the flowers fall on lines that are aligned in five principal directions, both in the scanning tunneling microscopy (STM) images and in the bulkterminated model (Fig. 2). STM data show that the pentagons defined by the starfish always have all five edges parallel to these lines, and the model shows that this is true for the empty pentagons of the substrate as well. Hence, the orientation of the starfish is consistent with the orientation of the pentagons, when the five principal directions defined by the substrate flowers comprise the frame of reference. This geometric argument is true, regardless of whether the two pentagons are overlaid or are rotated by $\pi / 5$ with respect to each 
other-their sides will be parallel in both cases. However, we will show below that the latter orientation is most probable.

The center holes of the empty pentagons vary in depth from plane to plane, from 0.76 to $1.99 \AA$, based upon the bulk structure. The starfish exhibit a shallower central depression which can also vary in depth, the maximum depth being $0.8 \AA$ below the starfish legs, based on STM measurements. This central feature is attributed to a deposited Al atom occupying the center hole, and hence being embedded slightly within the substrate plane. Furthermore, the purple pentagons have an edge length of $4.695 \AA$, which is slightly smaller than, but still comparable to, the measured edge length of $5.1 \pm 0.2 \AA$ for the pentagons defined by the starfish.

The identification of empty pentagons as reasonable surface sites for the starfish leads naturally to the model for Al nucleation and growth illustrated schematically in Fig. 3a-c. We postulate that a deposited $\mathrm{Al}$ atom diffuses across the surface until it drops into the hole at the center of an empty substrate pentagon, where it becomes trapped (Fig. 3b). This central atom stabilizes five more $\mathrm{Al}$ atoms around its periphery, as shown in Fig. $3 \mathrm{c}$. The stability of $\mathrm{Al}$ atoms at these five sites is made plausible by the fact that these sites are very near to $\mathrm{Al}$ lattice sites, i.e. sites that $\mathrm{Al}$ atoms would occupy if the entire upper layer were filled as it would be in the bulk quasicrystal. However, as noted above, the lateral separation of the Al atoms in the starfish is slightly larger than the separation of true lattice sites. This can be explained by the fact that the central $\mathrm{Al}$ atom occupies a site that would remain vacant in the bulk structure; hence, it 'pushes out' the surrounding $5 \mathrm{Al}$ atoms slightly, relative to their bulk positions. 
The observations described up until now hold true over the majority of the surface area. However, on a few terraces, Al growth is strikingly different--the Al forms fewer but larger, smoother islands, with no apparent fivefold symmetry. These terraces tend to be

bordered by 2.4 and $4.0 \AA$ steps. ${ }^{2}$ In the bulk structure model, planes associated with $2.47 \AA$ steps, and some of those associated with $4.0 \AA$ steps, contain no empty pentagons. Hence, the lack of starfish on these terraces is consistent with the idea that empty pentagons are necessary to engender the starfish.

As coverage increases, the film roughens rapidly. Once formed, the starfish do not grow laterally, but rather vertically. This is probably due to a combination of high interfacial energy (high strain) for an extended pseudomorphic Al layer on a quasicrystalline substrate, and low surface energy of the substrate. The apparent low surface energy of quasicrystals has long been noted. ${ }^{10}$ Both of these factors-high interfacial energy, and low substrate surface energy-would favour rough growth. ${ }^{11,12}$

In conventional thin films, nucleation and growth leads to a distribution of island sizes. In order to use these islands in various nanotechnologies, it is often desirable to make their size as uniform as possible. Indeed, significant effort has been expended to this end. It is therefore remarkable that the present system naturally forms very small, monodisperse islands, presumably due to the combined influence of interfacial and surface energies. In summary, we postulate that Al forms pseudomorphic starfish on the fivefold surface of icosahedral Al-Cu-Fe. The starfish nucleate at specific types of sites on the laterally bulkterminated quasicrystalline surface. The nucleation event consists of a diffusing $\mathrm{Al}$ atom dropping into an empty site at the center of a substrate pentagon. Growth consists of the 
addition of 5 Al atoms in (nearly) lattice sites. These 6-atom starfish do not grow laterally as coverage increases, leading to islands of uniform size, and to early roughening.

\section{Acknowledgements}

This work was supported by the Director, Office of Science, Office of Basic Energy Science, Materials Science Division of the U. S. Department of Energy, and by the EPSRC of the United Kingdom.

\section{References}

1. Janot, C. Quasicrystals: A Primer (eds. Humphreys, C. J., Hirsch, P. B., Mott, N. F. \&

Brook, R. J.) (Clarendon Press, Oxford, 1992).

2. Cai, T. et al. Structural aspects of the fivefold quasicrystalline Al-Cu-Fe surface from STM and dynamical LEED studies. Surface Science, in press (2001).

3. Thiel, P. A., Jenks, C. J. \& Goldman, A. I. in Physical Properties of Quasicrystals (ed. Stadnik, Z.) 327 (Springer-Verlag, Berlin, 1998).

4. Ledieu, J. et al. Tiling of the five-fold surface of Al 70 Pd 21 Mn 9. Surface Sci. Lett. 492, L729-L734 (2001).

5. Cai, T., Fournée, V., Lograsso, T., Ross, A. R. \& Thiel, P. A. An STM Study of the Atomic Structure of the Icosahedral AI-Cu-Fe Fivefold Surface. Phys. Rev. B: Rapid Communications, submitted (2001).

6. Gierer, M. et al. The Surface Structure of a Fivefold AIPdMn Quasicrystal: Dynamical Low-energy Electron Diffraction Analysis. Phys. Rev. Lett. 78, 467-470 (1997). 
7. Gierer, M. et al. Fivefold surface of quasicrystalline AlPdMn: Structure determination using low-energy-electron diffraction. Phys. Rev. B 57, 7628-7641 (1998).

8. Capitan, M. J., Alvarez, J., Joulaud, J. L. \& Calvayrac, Y. Characterization of the fivefold plane surface of an $\mathrm{Al}_{70.4} \mathrm{Pd}_{21.4} \mathrm{Mn}_{8.2}$ quasi-crystal by means of surface $\mathrm{X}$-ray diffraction. Surface Science 423, L251-L257 (1999).

9. Boudard, M. et al. Neutron and x-ray single-crystal study of the AlPdMn icosahedral phase. J. Phys. Condens. Matter 4, 10149-10168 (1992).

10. Dubois, J. M. et al. Quasicrystalline coatings with reduced adhesion for cookware. Ann. Chim. Fr. 19, 3-25 (1994).

11. Bauer, E. Phaenomenologische Theorie der Kristallabscheidung an Oberflaechen, I and II. Z. Kristallogr. 110, 372 and 395 (1958).

12. Bauer, E. \& van der Merwe, J. H. Structure and growth of crystalline superlattices: From monolayer to superlattice. Phys. Rev. B33, 3657 (1986). 


\section{Figure Captions}

Figure 1. Scanning tunneling micrograph $\left(45 \times 45 \mathrm{~nm}^{2}\right)$. Blue: deposited Al. Red: substrate. Rectangle with contents in gray: image in different contrast, chosen to reveal the flower structure of the nascent substrate. Rectangle to right of main figure: Expeanded views of two selected starfish.

Figure 2. Atomic positions in a plane $\left(10 \times 10 \mathrm{~nm}^{2}\right)$ from bulk model. Filled circles: $\mathrm{Al}$ atoms. Unfilled circles: $\mathrm{Fe}$ atoms. Hatched pentagons: examples of pentagons with internal atoms in two possible orientations. Black pentagons: examples of smaller pentagons with two possible orientations. Light gray pentagons: all the empty pentagons in the plane. Crossing lines: five principle directions lining up the centers of flowers on the substrate.

Figure 3. Schematic of the mechanism of starfish formation. Solid circles: atoms on the substrate. Hatched circles: deposited $\mathrm{Al}$ atoms. (a) The empty pentagon represents the nucleation sites on the substrate. (b) One deposited Al atom drops into the hole at the center of the empty pentagon and gets trapped. (c) Five more deposited $\mathrm{Al}$ atoms are stabilized at the periphery of the empty pentagon and form the starfish. 


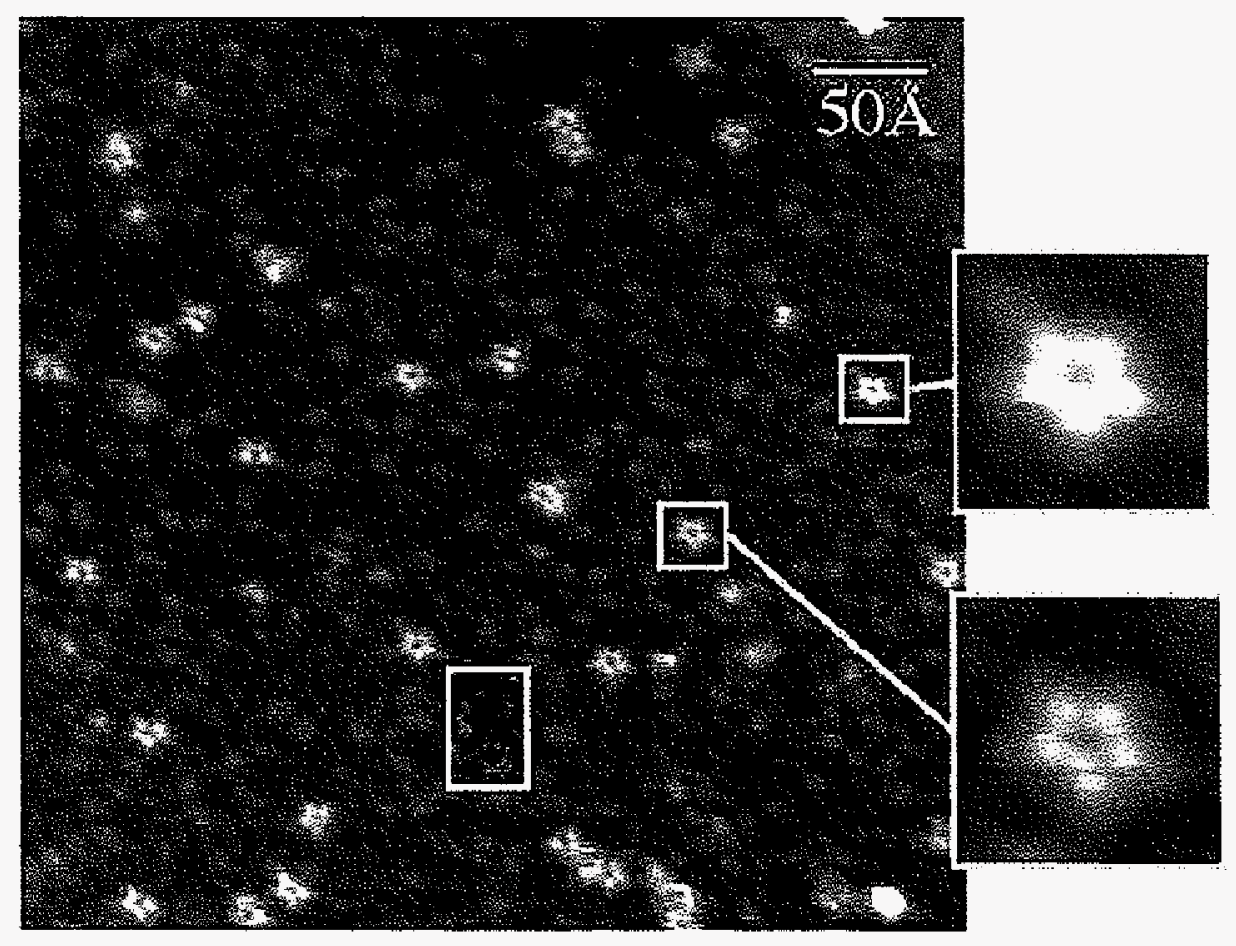

Figure 1 


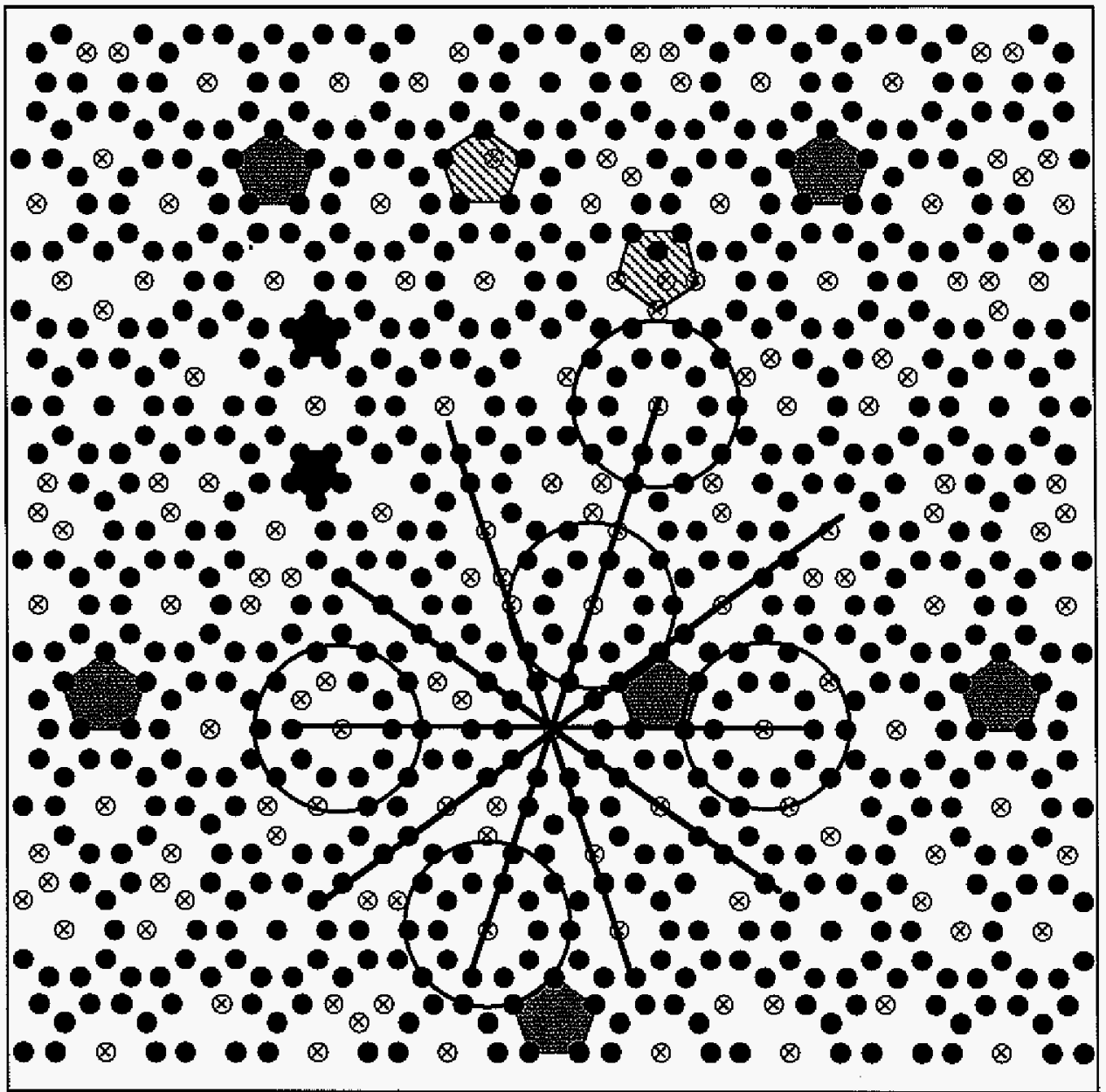

Figure 2 


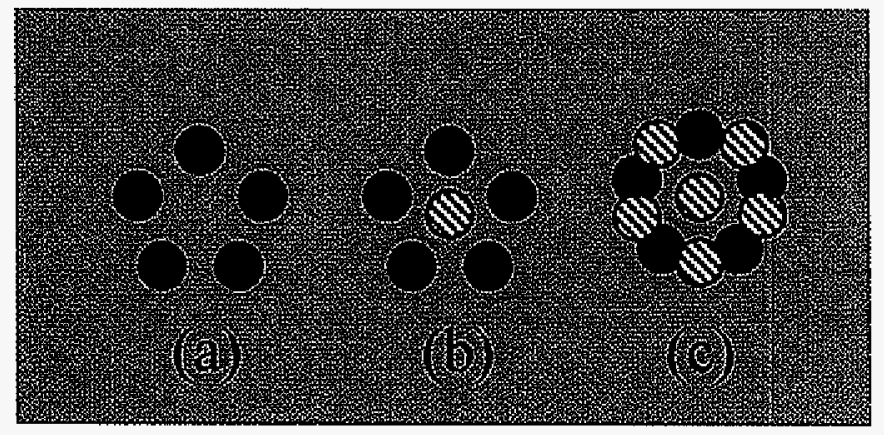

Figure 3 


\section{GENERAL CONCLUSIONS}

We have investigated the atomic-scale structure of the fivefold surface of an i-AlCu-Fe quasicrystal by STM imaging and a special dynamical LEED analysis. In addition, we have studied the nucleation and growth of aluminum thin films on the quasicrystalline surface of $\mathrm{i}-\mathrm{Al}-\mathrm{Cu}-\mathrm{Fe}$ by STM. The main conclusions that can be drawn from the work described in this dissertation are given below.

\section{The clean surface}

From dynamical LEED calculations, we conclude,

a) The surface tends to form between different groups of closely-spaced planes by splitting the quasicrystal through larger interplanar spacings that separate those groups.

b) The closely-spaced planes can be sorted into three sets, with 3,5 and 9 (sometimes 11) planes, corresponding to step heights of $2.47 \AA, 3.99 \AA$ and $6.47 \AA$, respectively.

From STM measurements, we conclude,

a) The step heights adopt (primarily) two values of $4.0 \AA$ and $6.4 \AA$, while a stepheight of $2.5 \AA$ is found with much less frequency.

b) The spatial distribution of the two primary stepheights along the fivefold axis does not follow a Fibonacci sequence, thus breaking the ideal bulk-like quasicrystalline layer stacking order perpendicular to the surface. The screw dislocations observed in the STM images also indicate the imperfect quasiperiodic order. 
c) The atomically-flat terraces feature many ten-petal "flowers" with internal structure. Such flower-patterns and their arrangements on the terrace can be associated with features on dense atomic planes generated from two-dimensional cuts of a bulk model based on X-ray and neutron diffraction experiments.

The results from dynamical LEED calculations and STM observations agree well with each other. These results suggest that the fivefold surface of i-Al-Cu-Fe is bulkterminated. It exhibits a quasicrystalline layered structure, but with stacking defects.

\section{The nucleation and growth of Al thin films on $\mathrm{i}-\mathrm{Al}-\mathrm{Cu}-\mathrm{Fe}$ fivefold surface}

a) At low coverage, Al adatoms nucleate at specific sites on the substrate, and form pentagonal "starfish" with uniform size and specific orientation. We proposed a nucleation model to interpret the formation of the starfish.

b) With increasing coverage, the Al thin film does not follow layer-by-layer growth, which is consistent with the low surface energy of quasicrystalline material.

c) Upon deposition, a small fraction of terraces accommodate the growth of smoother and bigger Al islands compared to their surrounding terraces. Such "special" terraces might exhibit higher surface energy. 


\title{
APPENDIX A. STRUCTURAL ASPECTS OF THE THREE-FOLD SURFACE OF ICOSAHEDRAL AL-PD-MN
}

\author{
A paper published in Surface Science Letters \\ D. Rouxel, T. -H. Cai, C. J. Jenks, \\ T. A. Lograsso, A. Ross, and P. A. Thiel
}

\begin{abstract}
We report the first STM study of a threefold surface of an icosahedral quasicrystal. We find that a rough, cluster-dominated structure evolves into a terrace-step morphology, with increasing temperature. The terraces display a fine structure whose long-range order is consistent with threefold symmetry. The fine structure includes small, deep holes. The steps can be very straight, serving to bound equilateral triangles (or portions thereof). These straight steps can cut directly across meandering step edges, superimposing a triangular "shadow" upon the other terrace-step landscape. The data suggest that the triangles grow outward from a special type of central point. The triangles may represent the initial stages of facetting.
\end{abstract}

Keywords: Aluminum; Alloys; Metallic surfaces; Scanning tunneling microscopy (STM).

Quasicrystals are well-ordered, but aperiodic, intermetallics. They typically exhibit a rotational symmetry which is crystallographically-forbidden. It is of interest to determine whether, and in what ways, their surfaces resemble their bulk. On one hand, this issue is 
motivated both by practical interest - a desire to understand the origins of their low friction and reduced adhesion. On the other hand, this issue is motivated by fundamental curiosity - a desire to understand whether the forces which stabilize a three-dimensional structure remain in effect at its two-dimensional truncation.

Many experimental techniques can probe surface structure. For a material as complex as a quasicrystal, most techniques will provide only a partial answer, and a complete picture will evolve only after many different types of experiments. In this paper, we apply one technique, scanning tunneling microscopy (STM). This technique can provide information both about local and long-range structure. For quasicrystalline surfaces, however, it has not yet provided atomic-scale resolution nor chemical speciation.

Surfaces of quasicrystals can be prepared in a variety of ways. The most common, sputtering and annealing, can yield terrace-step features when the surfaces have compositions close to that of the bulk. (Cleavage can produce much rougher surfaces. [1]) Scanning tunneling microscopy studies of the terrace-step-type surfaces have thus far been reported only for fivefold surfaces of icosahedral materials[2-9] and tenfold surfaces of decagonal materials[9-12]. These studies have often revealed small local structures, e.g. pentagons, which are consistent with the symmetry of the zone axis perpendicular to the surface. In some cases, the STM has even revealed a longer-range order on the terraces, again consistent with the symmetry expected for the bulk termination.

However, quasicrystals also possess other high-order symmetry axes, and surfaces perpendicular to these axes have not yet been studied with STM, perhaps because they are less exotic than the crystallographically-forbidden fivefold and tenfold symmetries. Nonetheless, they are essential to build a complete database of information on 
quasicrystalline surfaces. In this paper, we report an STM study of the threefold surface of icosahedral Al-Pd-Mn. While this surface has not been studied previously with STM, it has been studied with low-energy electron diffraction (LEED).[13-15] The latter technique has shown that a quasicrystalline(-like) surface can be prepared, after sputtering, by prolonged annealing in the range $650-850 \mathrm{~K}$. At lower annealing temperatures, a cubic (111) overlayer develops. At even lower temperatures, an unidentified crystalline phase develops.[14] However, the crystalline phases are not the focus of the present work. Instead we concentrate on the temperature regime which produces the quasicrystalline(-like) LEED pattern.

The experiments were performed with equipment, and under conditions, similar to those described previously in a study of the fivefold surface.[5] The tunneling voltage was $1.0 \mathrm{~V}$, and the current was 0.3 to $0.6 \mathrm{nA}$. Use of lower tunneling currents, down to $0.03 \mathrm{nA}$, did not produce a qualitative difference in the images.

Two different samples were used. Each of these samples went through two cycles of being polished and then examined with STM. Hence, in total, the experiments were performed on four different surfaces. After any given annealing temperature, a variety of structures could be found on the surface, based upon examination of many images at different surface areas, over all four of these surfaces. In other words, the surfaces are heterogeneous. However, it is our strong impression that the surfaces generally evolve according to the sequence described below, even though different regions may not change exactly in parallel. The sample was typically annealed at the stated temperature for one-half to four hours, then re-cooled to $300 \mathrm{~K}$ for imaging. A threefold LEED pattern was observed 
in parallel with the STM measurements. The LEED optics were too poor, however, to resolve significant structure in the pattern.

Fig. 1 shows two examples of rough surfaces obtained at the lowest annealing temperatures $(800-850 \mathrm{~K})$. Fig. 1a and $1 \mathrm{c}$ are micrographs, while $1 \mathrm{~b}$ and $1 \mathrm{~d}$ are corresponding line scans. Both surfaces have cluster-like structures, the clusters being about $10 \mathrm{~nm}$ in diameter. However, the clusters are better-separated in Fig. 1a than in 1c, which leads to higher roughness in the former case. This is illustrated by the line scans, Fig. $1 \mathrm{~b}$ and 1c. The values of the root-mean square roughness are $2.3 \mathrm{~nm}$ and $0.96 \mathrm{~nm}$, respectively. (Corresponding arithmetic means are 1.9 and 0.77 .) These clusters may represent the cubic structure, although they may also (by analogy with suggestions for the fivefold surface[8, 16]) represent a quasicrystalline intermediate between the rough cubic phase and the quasicrystalline terrace-step structure. We note that in this temperature range, the LEED pattern is already that of the quasicrystalline(-like) surface.[14, 15]

At slightly higher temperatures, or longer annealing times, the clusters become more isolated and terraces emerge. Both features can co-exist. This is illustrated in Fig. 2a-b. Images are poor for such surfaces because the remaining clusters tend to streak and change the tip characteristics during the scan. The step edges are rough and meandering, and may be pinned by clusters.

Around $875-900 \mathrm{~K}$, the clusters disappear. The step edges are still very rough. This is shown by the large-scale image of Fig. 3a. The terraces exhibit a fine structure, which is shown in Fig. 3b. The fine structure appears random upon visual inspection. The most pronounced features are small holes, which appear black in Fig. 3b. The line scans (Fig. 3c) reveal that these holes are about $0.2 \mathrm{~nm}$ deep, while the rest of the surface has a peak-to- 
peak corrugation of about $0.1 \mathrm{~nm}$. (The root-mean square roughness is $0.06 \mathrm{~nm}$ and the arithmetic mean is $0.04 \mathrm{~nm}$.) The holes are about $1-2 \mathrm{~nm}$ wide. It should be noted that STM images the electronic density contours, which may or may not reflect nuclear positions. Hence, the "corrugation" and "holes" on the terraces may actually be part of the electronic topography, rather than the atomic topography, or some convolution of the two.

When Fig. $3 b$ is corrected for distortion, its Fourier transform exhibits six-fold symmetry, hence indicating a degree of order in the real-space structure. The sixfold autocorrelation function of Fig. $3 \mathrm{~d}$ confirms this. Note that both the Fourier transform and the autocorrelation introduce an inversion center, so that a threefold structure in real-space becomes sixfold after each manipulation. Hence, both manipulations are consistent with the observation of a threefold pattern in LEED under similar conditions.[15]

At $900 \mathrm{~K}$, straight step edges emerge. They usually coexist with the meandering step edges. The straight step edges are the boundaries of equilateral triangles, or portions of such triangles. The straight step edges can actually cut across the meandering step edges. This produces an image such as Fig. 4a, where the triangle looks like a shadow cast upon the underlying landscape. This intriguing type of structure is illustrated also in Fig. 4b. Smaller triangles are illustrated in Fig. 4c. The triangles can be either depressions in, or protrusions from, the surface. The large triangles in Fig. 4a-b are depressions.

As shown in Fig. 4a-b, one often finds several triangles-or sections of triangles-nested concentrically. The straight step edges are always parallel, no matter how large the scale of the image. This is especially striking in Fig. $4 c$, where three non-overlapping triangles are shown, all with parallel step edges. Furthermore, only three step orientations are ever observed. Hence, the orientations of the straight steps must be controlled by the 
fundamental threefold symmetry of the zone axis. This is consistent with the relative orientation of the triangles in STM, and the LEED pattern observed in parallel experiments (in the same chamber).

Higher temperatures were not accessible due to phase decomposition of the sample.

We can speculate about the mechanism and driving force for evolution of the straight step edges. The fact that they form closed triangles, and even concentric triangles, rather than randomly-intersecting lines, suggests strongly that they grow outward from a point of origin which is somehow unique. One can imagine that the small triangles in Fig. $4 \mathrm{c}$ could be the precursors to the larger ones in Fig. 4a,b. The small triangles might grow larger with more severe annealing, one of the triangles eventually overtaking and consuming the others. The small triangles do seem to have something at their centers, as can be seen in Fig. 4c. At this time, however, we cannot determine the nature of the centers of the small triangles. It may be that the triangles are the first stages of faceting, with their straight edges perpendicular to the three fivefold axes. In a previous study, we have shown that the threefold surface does tend to facet, toward the fivefold axes and toward an unidentified direction.[15]

There are some indications that the triangles are related to the black holes. The density of the holes seems to increase between $800 \mathrm{~K}$ and $900 \mathrm{~K}$. At a temperature slightly lower than $900 \mathrm{~K}$, just before triangles appear, we found at different places a density of holes so high that they seem to outline the edges of triangles. Thus, we speculate that the holes may be the precursors to the small triangles, and the small triangles in turn may be the precursors to the larger ones.

It is interesting to compare these observations to those for the fivefold surface, prepared under similar conditions. There, a progression was also observed, from a rough, 
cluster-dominated structure to a terrace-step structure, with increasing temperature. However, the step edges in the terrace-step structure were always rough (meandering); no straight step edges were ever observed. For the fivefold surface, a fine structure was also observed on the steps, with a clear rotational symmetry and long-range order, as revealed by the Fourier transform and autocorrelation function. However, the peak-to-peak corrugation was about $0.08 \mathrm{~nm}$; the small deep holes were not observed. Hence, the threefold surface differs from the fivefold, for comparable conditions of preparation, in two main ways: First, it develops straight step edges, which encompass equilateral triangles; and second, it exhibits $0.2 \mathrm{~nm}$-deep depressions in the terrace fine structure, which are deeper than depressions observed on the fivefold surface, under comparable tunneling conditions.

In summary, this STM study of the threefold surface of icosahedral Al-Pd-Mn has shown the same general trend of morphological development as for the fivefold surface: a rough, cluster-dominated structure evolves into a terrace-step configuration. The terraces display a fine structure whose long-range order is consistent with threefold symmetry. However, the fine structure on the terraces is rougher than on the fivefold surface, exhibiting holes that are $0.2-\mathrm{nm}$ deep. Furthermore, the steps can be very straight, serving to bound equilateral triangles (or portions thereof) whose edges are always parallel. These straight steps can superimpose upon the meandering steps. The data suggest that the triangles grow outward from a special central point. These intriguing observations provoke further study.

\section{Acknowledgments}

This work was supported by the Ames Laboratory, which is operated for the U.S. Department of Energy by Iowa State University under Contract No. W-7405-Eng-82, and 
by Programme International de Coopération Scientifique (PICS) under Grant No. 545 NSFCNRS.

\section{References}

1. P. Ebert, M. Feuerbacher, N. Tamura, M. Wollgarten, K. Urban, Phys. Rev. Lett. 77 (1996) 3827 .

2. T. M. Schaub, D. E. Bürgler, H.-J. Güntherodt, J. B. Suck, Phys. Rev. Lett. 73 (1994) 1255.

3. T. M. Schaub, D. E. Bürgler, H.-J. Güntherodt, J.-B. Suck, Z. Phys. B 96 (1994) 93.

4. T. M. Schaub, D. E. Bürgler, H.-J. Güntherodt, J. B. Suck, M. Audier, Appl. Phys. A 61 (1995) 491.

5. Z. Shen, C. Stoldt, C. Jenks, T. Lograsso, P. A. Thiel, Phys. Rev. B (1999) submitted.

6. G. Cappello, A. Dechelette, F. Schmithüsen, J. Chevrier, F. Comin, A. Stierle, V. Formoso, M. de Boissieu, T. Lograsso, C. Jenks, D. Delaney, "Characterization and properties of the AIPdMn 5 surface," in: J. M. Dubois, P. A. Thiel, A. P. Tsai, K. Urban (Ed.) MRS Proceedings: Quasicrystals, Vol. 553, MRS, Boston, 1999, p. 243.

7. J. Ledieu, A. Munz, T. Parker, R. McGrath, R. D. Diehl, D. W. Delaney, T. A. Lograsso, Surface Science (1998)

8. J. Ledieu, A. W. Munz, T. M. Parker, R. McGrath, R. D. Diehl, D. W. Delaney, T. A. Lograsso, "Clustered, terraced, and mixed surface phases of the $\mathrm{Al}_{70} \mathrm{Pd}_{21} \mathrm{Mn} 9$ quasicrystal," in: J. M. Dubois, P. A. Thiel, A.-P. Tsai, K. Urban (Ed.) MRS Proceedings: 
Quasicrystals, Materials Research Society Symposium Proceedings Vol. 553, Materials Research Society, Warrendale, Pennsylvania, 1999, p. 237.

9. R. S. Becker, A. R. Kortan, F. A. Thiel, H. S. Chen, J. Vac. Sci. Technol. B 9 (1991) 867.

10. R. S. Becker, A. R. Kortan, "Scanning tunneling microscopy studies of quasicrystals," in: D. P. DiVincenzo, P. Steinhardt (Ed.) Quasicrystals: The State of the Art, World Scientific Publishing Co., Singapore, 1991, p. 111.

11. A. R. Kortan, R. S. Becker, F. A. Thiel, H. S. Chen, Phys. Rev. Lett. 64 (1990) 200.

12. A. R. Kortan, R. S. Becker, F. A. Thiel, H. S. Chen, "Structure of decagonal quasicrystals," in: P. Jena, S. N. Khanna, K. Rao (Ed.) Physics and Chemistry of Finite Systems: From Clusters to Crystals, Vol. 1, Kluwer Academic Publishers, Dordrecht, 1992, p. 29.

13. C. J. Jenks, P. J. Pinhero, Z. Shen, T. A. Lograsso, D. W. Delaney, T. E. Bloomer, S.-L. Chang, C.-M. Zhang, J. W. Anderegg, A. H. M. Z. Islam, A. I. Goldman, P. A. Thiel, "Preparation of icosahedral AlPdMn and AlCuFe samples for LEED studies," in: S.

Takeuchi, T. Fujiwara (Ed.) Proceedings of the 6th International Conference on Quasicrystals (ICQ6), World Scientific, Singapore, 1998, p. 741.

14. Z. Shen, M. J. Kramer, C. J. Jenks, A. I. Goldman, T. Lograsso, D. Delaney, M. Heinzig, W. Raberg, P. A. Thiel, Phys. Rev. B 58 (1998) 9961.

15. Z. Shen, W. Raberg, M. Heinzig, C. J. Jenks, V. Fournée, M. A. V. Hove, T. A. Lograsso, D. Delaney, T. Cai, P. C. Canfield, I. R. Fisher, A. I. Goldman, M. J. Kramer, P. A. Thiel, Surface Science (1999) submitted. 16. J. Chevrier, (1999) private communication. 


\section{Figure Captions}

Figure 1. Two examples of cluster-dominated surface structure. (a) STM image, obtained after annealing at $850 \mathrm{~K}$ for 1 hour. Total area: $400 \times 400 \mathrm{~nm}$. (b) Line scans corresponding to Fig. 1a. (c) STM image, obtained after annealing at $800 \mathrm{~K}$ for 1 hour. Total area: $100 \times 100 \mathrm{~nm}$. (d) Line scans corresponding to Fig. 1c.

Figure 2. Two examples of coexisting cluster and terrace-step structures. (a) STM image, obtained after annealing at $800 \mathrm{~K}$ for 4 hours. Total area: $300 \times 300 \mathrm{~nm}$. (b) STM image, obtained after annealing at $800 \mathrm{~K}$ for 2 hours. Total area: $90 \times 90 \mathrm{~nm}$.

Figure 3. Terrace-step structure with meandering steps only. (a) STM image, obtained after annealing at $900 \mathrm{~K}$ for 0.5 hours. Total area: $80 \times 80 \mathrm{~nm}$. (b) STM image of the terrace fine structure, obtained after annealing at $875 \mathrm{~K}$ for 2 hours. Total area: $50 \times 50$ nm. (c) Line scans corresponding to Fig. 3b. (d) Autocorrelation transform of Fig. 3b.

Figure 4. Three examples of terrace-step structures, where meandering and straight step edges coexist. All images obtained after annealing at $900 \mathrm{~K}$ for 0.75 hours. (a) Total area: $140 \times 140 \mathrm{~nm}$. (b) Total area: $150 \times 150 \mathrm{~nm}$. (c) Total area: $60 \times 60 \mathrm{~nm}$. 

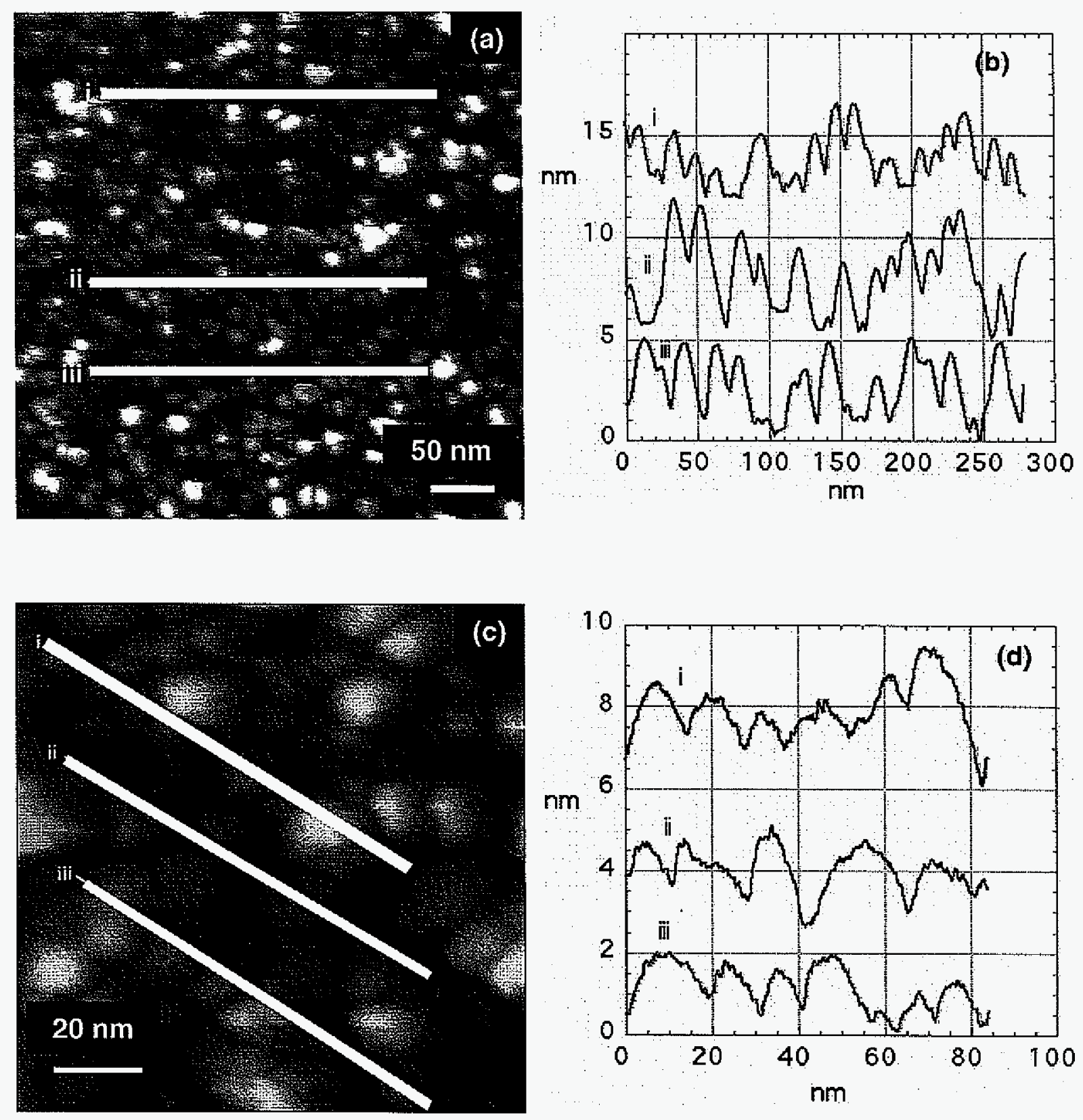

Figure 1 

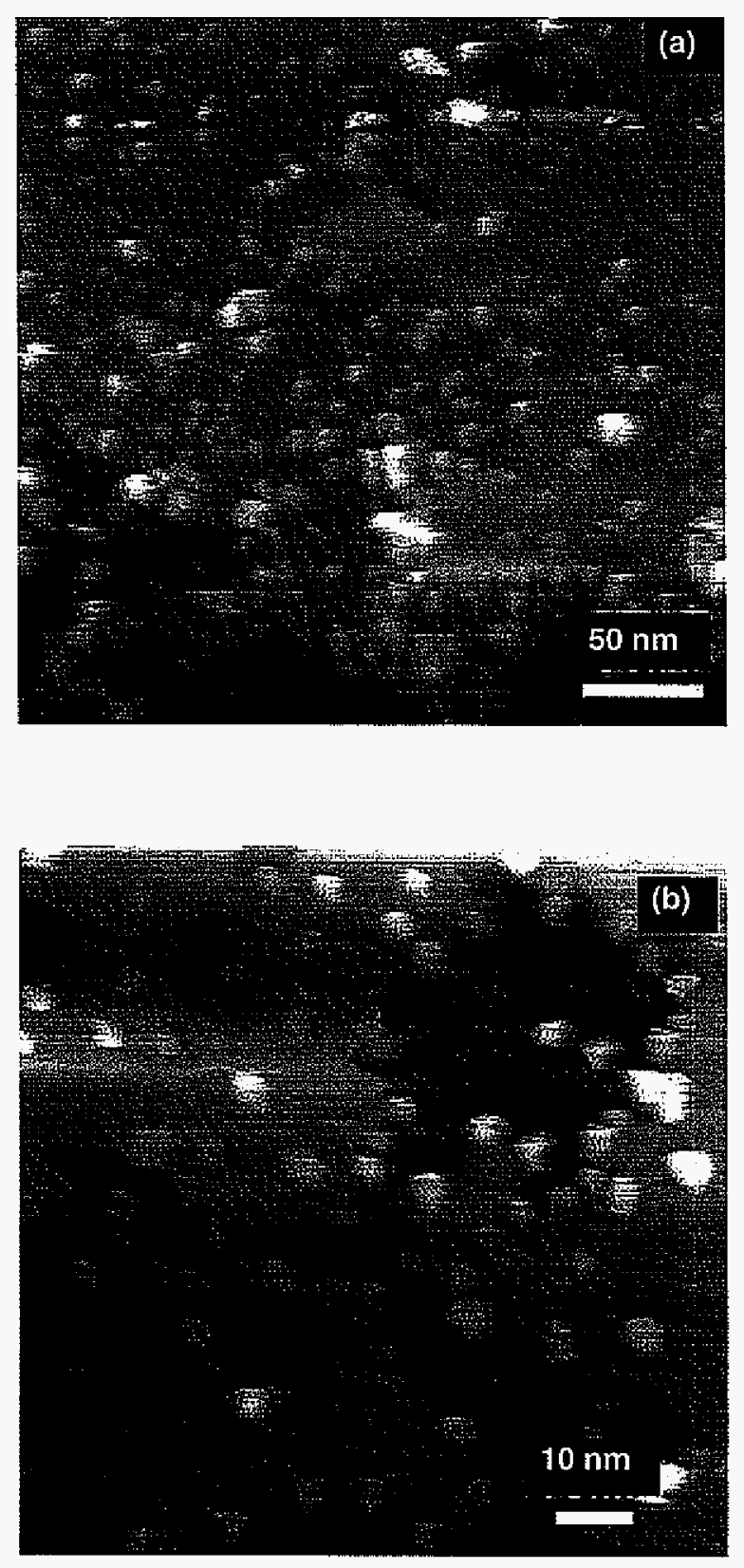

Figure 2 

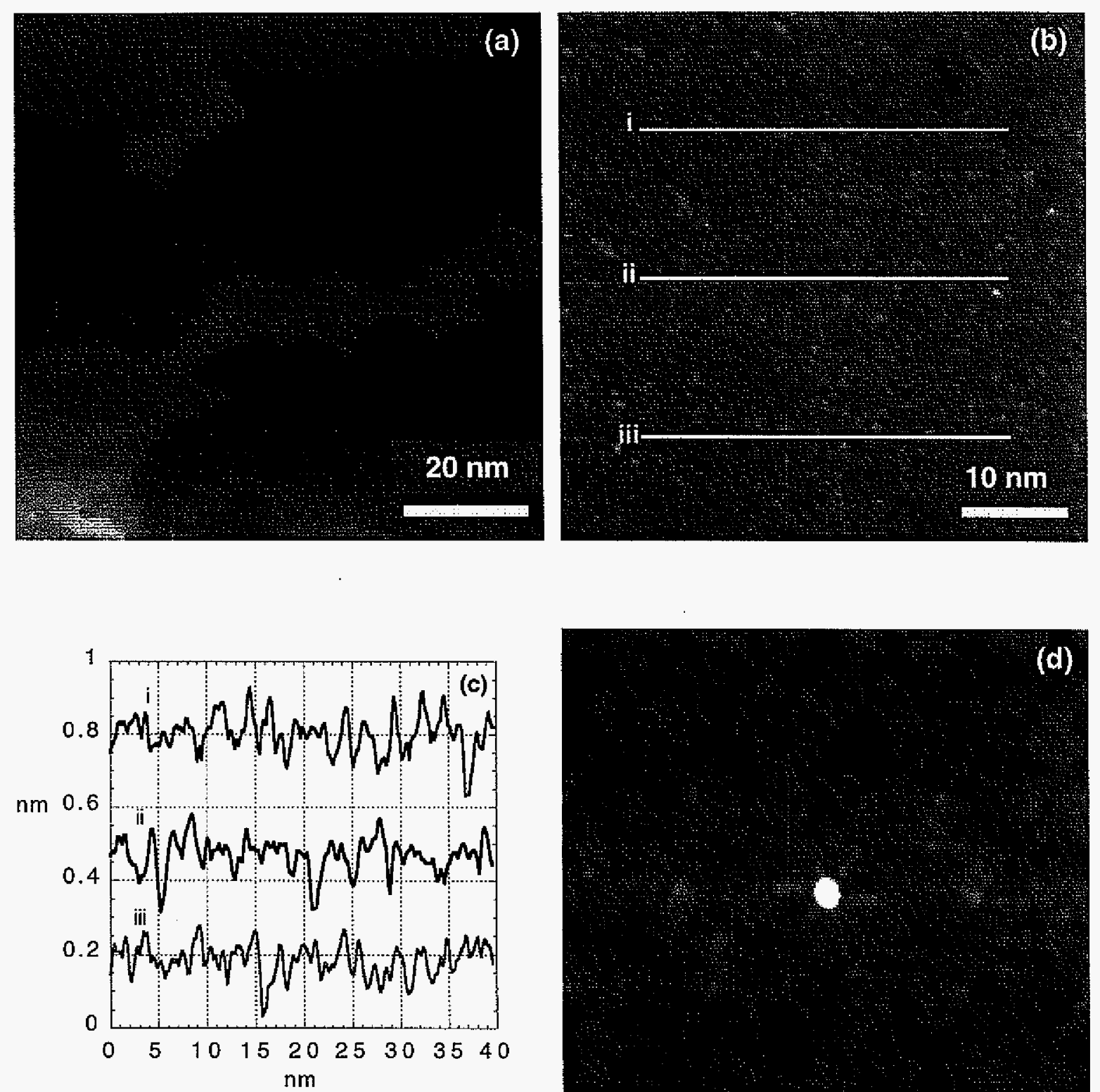

(d)

Figure 3 

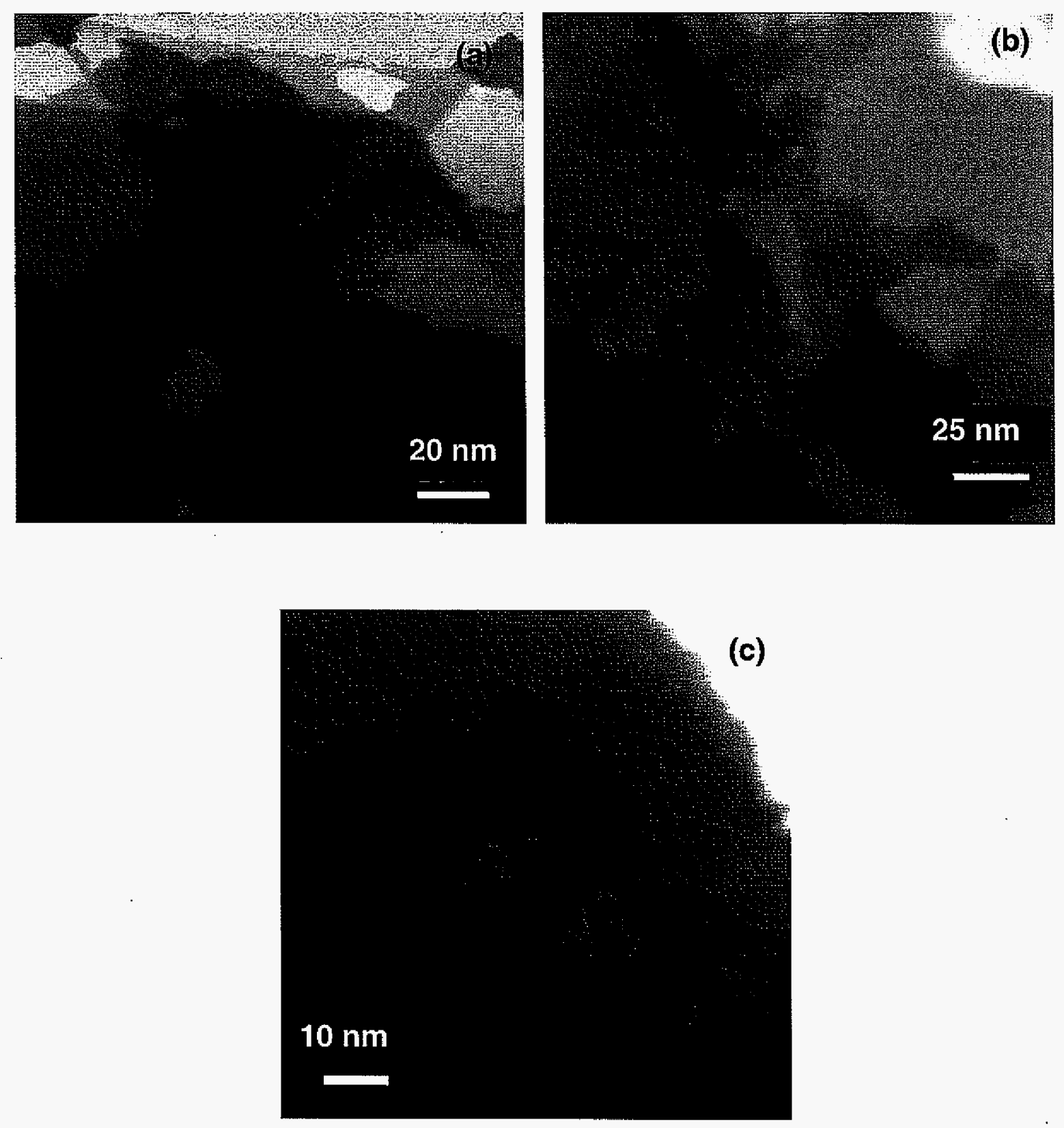

Figure 4 


\section{APPENDIX B. THE ALUMINUM EVAPORATOR}

To design a long-lived aluminum evaporator in UHV with reproducible deposition flux, one needs to consider several facts. a) Aluminum has a sufficient vapor pressure only at temperatures far beyond its melting point. Operation at such high temperatures often makes the evaporator unacceptably gassy in UHV. b) Once the vapor pressure reaches a high value, it becomes a steep function of temperature. This challenges the stability of the deposition flux. c) Aluminum has a very low surface tension at operating temperatures and will creep away from the initial evaporation point. d) Aluminum has a strong tendency to form alloys with other materials.

The aluminum evaporator described in this report is based on the design published in the paper "Long-lived aluminum evaporation source for controlled, reproducible deposition of clean ultrathin films under ultrahigh vacuum conditions" by J. Wytenburg et al. (J. Vac. Sci. Technol. A 10, Page 3597, 1992).

The general design of our aluminum evaporator is shown in the sketch in Fig. 1. The evaporator is mounted on a DN38 (2 3/4" o.d.) Conflat@ flange that contains a pair of electrical feedthroughs and a pair of thermocouple feedthroughs. The source is then enclosed in a water-shroud for cooling purposes during evaporation. The hole at the end of the shroud also helps the collimation. The shutter for the source is mounted on another port which is close to where the evaporator is mounted.

The source itself (see Fig. 2) consists of an $\mathrm{Al}_{2} \mathrm{O}_{3}$ tube (Scientific Instrument, Part NO. R19, 0.188-inch-o.d., 0.125-inch-i.d., 2.4-cm-long) into which a pure Al rod (0.121inch-diameter) is inserted. The $\mathrm{Al}$ rod is approximately half as long as the $\mathrm{Al}_{2} \mathrm{O}_{3}$ tube. The 
$\mathrm{Al}_{2} \mathrm{O}_{3}$ tube is then fit tightly into a $\mathrm{W}$ heating coil ( 7 turns of 0.1 -mm-diameter wire). In order for the $\mathrm{Al}$ rod to fit tightly in the $\mathrm{Al}_{2} \mathrm{O}_{3}$ tube, the $\mathrm{Al}$ rod is wrapped with Ta foil. An $\mathrm{Al}_{2} \mathrm{O}_{3}$ rod (Scientific Instrument, Part NO. R15) is inserted into the unfilled end of the tube. Pieces of Ta foil are smashed between the $\mathrm{Al}$ and $\mathrm{Al}_{2} \mathrm{O}_{3}$ rod to prevent the melted $\mathrm{Al}$ from creeping out the tube. Both ends of the $W$ coil are connected with thick Ta $(0.5 \mathrm{~mm}-$ diameter) wires which themselves are connected to the electronic feedthroughs by Barrel connectors. The thermocouple wires are spot-welded at the point where the Ta wire and the W coil meet to make good contact for temperature measurement. Although the thermocouple is not reading the temperature of the Al rod itself, it does give a good indication of the behavior of the aluminum source.

The source is operated by applying a constant voltage across the pair of electrical feedthroughs. The $\mathrm{W}$ coil is then resistively heated, in turn heating the $\mathrm{Al}_{2} \mathrm{O}_{3}$ tube and the encased $\mathrm{Al}$ rod. In order to limit the contamination during deposition to the lowest possible level, the evaporator has to be outgassed thoroughly with the shutter closed before operation. During each deposition run, we follow the same procedure to reach the operating power setting in order to yield a reproducible and constant evaporation rate at a certain setting. To do so, we allow five minutes for outgassing before we progressively increase the voltage by $5 \mathrm{~V}$ each time. Once we reach the final operating setting, we wait for ten minutes before starting deposition. The pressure normally remains below $6 \times 10^{-10}$ Torr during $\mathrm{Al}$ deposition.

The cleanliness of the deposited Al films is examined by Auger electron spectroscopy. No evidence of codeposition of other metals is found, and the contamination by background gases is below the detection limit of the instrument. 
To estimate the $\mathrm{Al}$ coverage, we deposit aluminum on an $\mathrm{Al}$ (111) surface and record the STM images. The coverage is estimated by calculating the fraction of the area covered by $\mathrm{Al}$ islands in the images. Within the coverage regime investigated in the work of $\mathrm{Al}$ deposition on $\mathrm{i}-\mathrm{Al}-\mathrm{Cu}-\mathrm{Fe}$ quasicrystalline surface described previously in this dissertation, the $\mathrm{Al}$ coverage is linear with dosing time. Table 1 summarizes the power settings as well as the estimated coverage for the aluminum evaporator with the geometry quoted in this report.

Table 1. Aluminum flux rate at different power settings

\begin{tabular}{|c|c|c|c|c|c|}
\hline \multicolumn{3}{|c|}{$\begin{array}{l}\text { Aluminum source } \\
\text { Power settings }\end{array}$} & \multirow{2}{*}{$\begin{array}{l}\text { Dosing time } \\
\text { (seconds) }\end{array}$} & \multirow{2}{*}{$\begin{array}{c}\text { Coverage } \\
\text { (ML) }\end{array}$} & \multirow{2}{*}{$\begin{array}{c}\text { Flux } \\
(\mathrm{ML} / \mathrm{s})\end{array}$} \\
\hline $\mathrm{U}(\mathrm{V})$ & $\mathrm{I}(\mathrm{A})$ & $\begin{array}{l}\text { Thermocouple } \\
\text { readings (mV) }\end{array}$ & & & \\
\hline \multirow[b]{2}{*}{10.0} & \multirow[b]{2}{*}{5.0} & \multirow[b]{2}{*}{20.2} & 30 & 0.04 & \multirow[b]{2}{*}{$1.7 \times 10^{-3}$} \\
\hline & & & 60 & 0.10 & \\
\hline 8.9 & 4.7 & 19.2 & 120 & 0.01 & $8.3 \times 10^{-5}$ \\
\hline 11.0 & 5.5 & 21.7 & 30 & 0.20 & $6.7 \times 10^{-3}$ \\
\hline
\end{tabular}




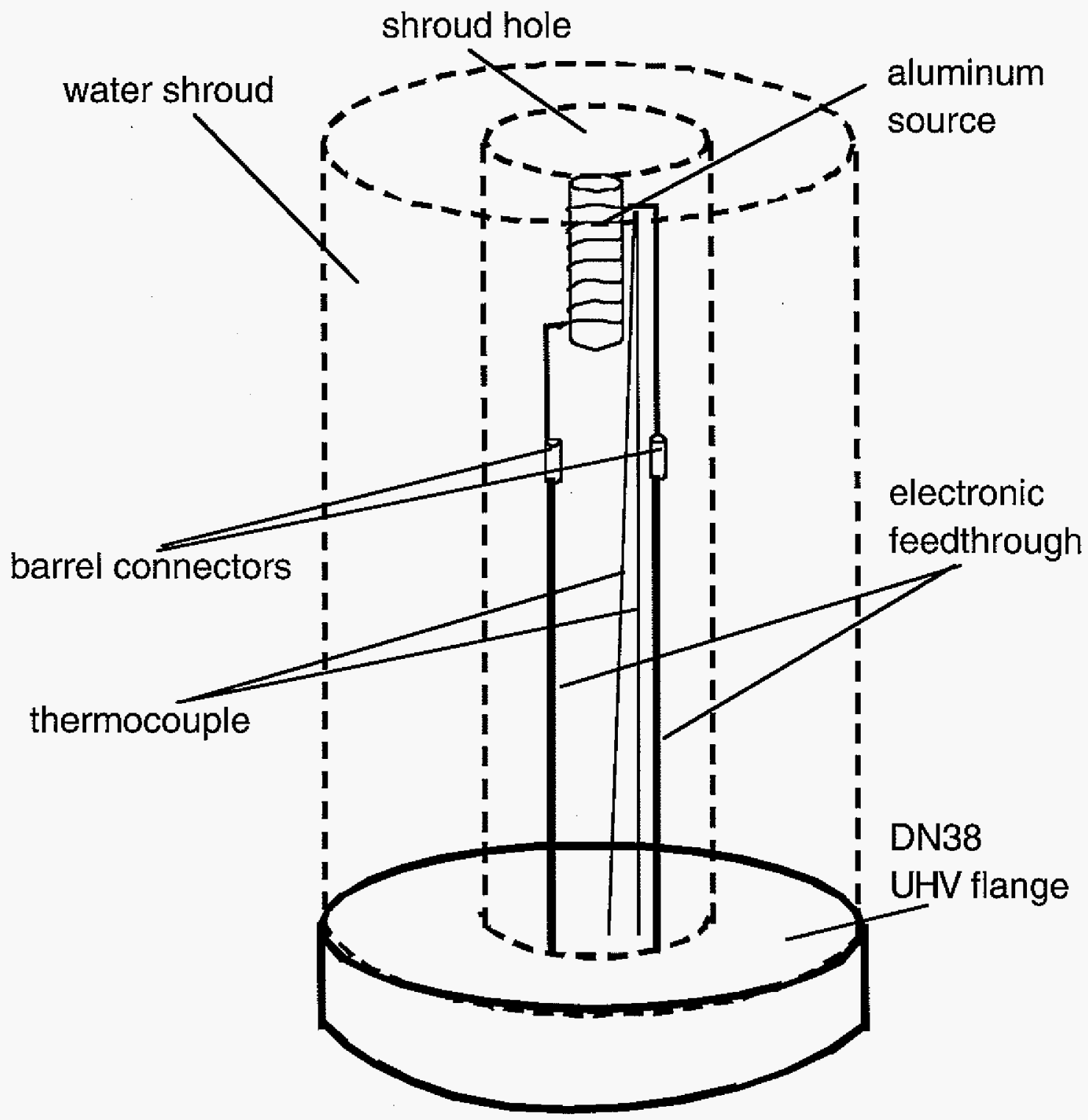

Figure 1. Sketch of general design of Al evaporator 


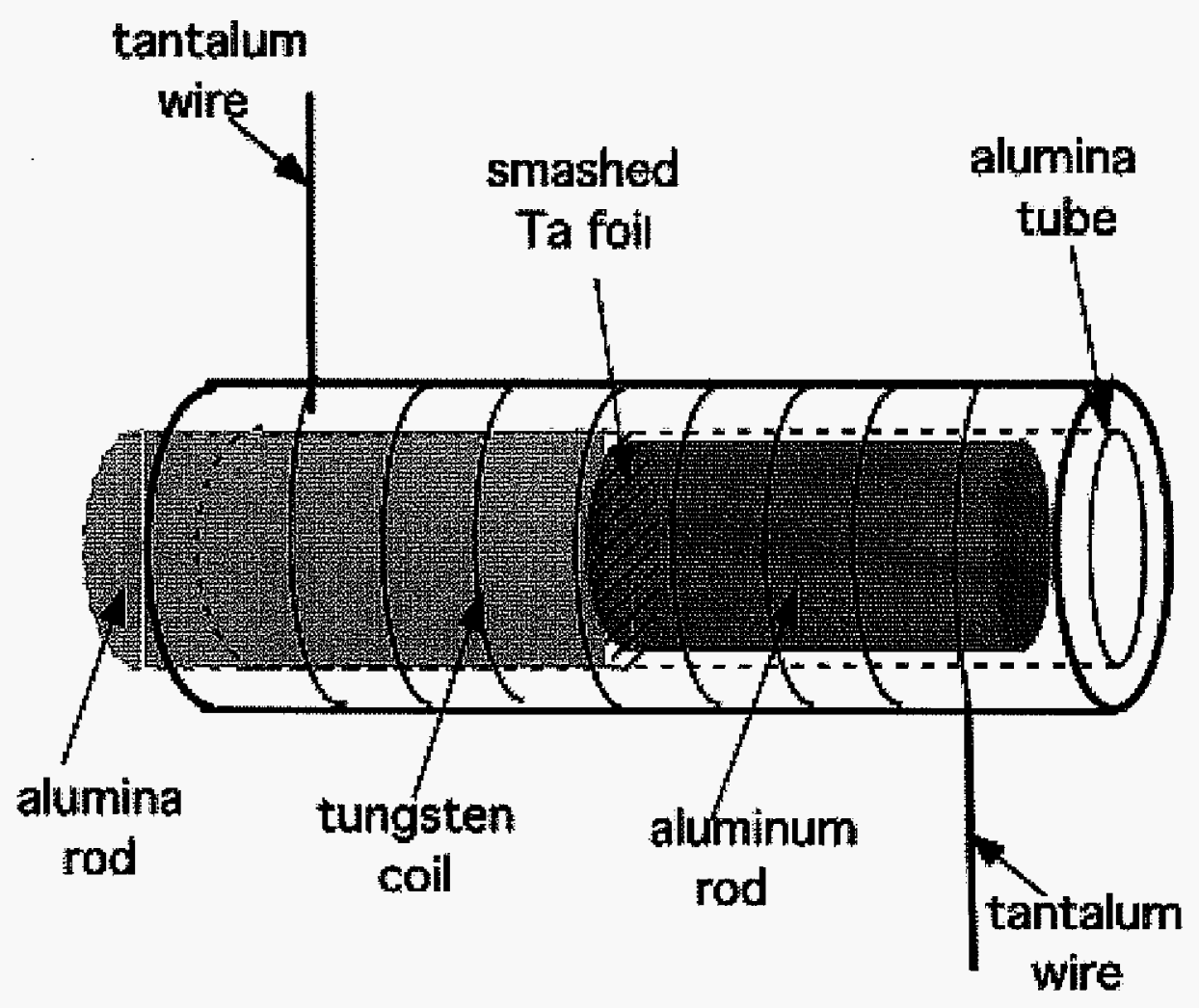

Figure 2. Sketch of $\mathrm{Al}$ evaporator source 


\title{
APPENDIX C: STRUCTURE OF ALUMINUM FILM DEPOSITED ON ICOSAHEDRAL AL-CU-FE FIVEFOLD SURFACE
}

\author{
T. Cai, J. Ledieu, V. Fournée, T. Lograsso, A. Ross, R. McGrath, P. Thiel
}

\begin{abstract}
Using scanning tunneling microscopy (STM), we investigate the nucleation and growth of Al film deposited on the fivefold surface of icosahedral (i-) Al-Cu-Fe quasicrystal at room temperature. At low coverages, the $\mathrm{Al}$ adatoms nucleate into pentagonal starfishislands, preserving the fivefold symmetry of the substrate. These starfish are of the same size and they adopt the same orientation on the same terrace as well as across different terraces. As coverage increases, the starfish grow vertically and the Al film roughens. Based on the analysis of experimental observations and the available bulk structure model, we identify the specific surface sites for the nucleation and develop the model of the formation of starfish-islands.
\end{abstract}

\section{Introduction}

In contrast to conventional crystals, quasicrystals do not have a long-range periodic order [1]. A number of experimental studies have confirmed that their bulk quasiperiodic structure is indeed maintained up to the surface [e.g. 2-4]. Recently, the topic of growing thin metallic film on quasicrystalline substrate has attracted particular interest for several reasons. For instance, it is a tempting tryout to establish a two-dimensional quasicrystalline overlayer consisting of one single species $[5,6]$. In addition, the structure of the growing thin 
metal film represents a geometric balance between the quasiperiodic structure of the substrate and the periodic bulk structure of the respective film material, and thus will possibly provide additional structural and chemical information on the quasicrystal substrate. Furthermore, it is of great interest to examine the structural transition at crystalline-quasicrystalline interface since quasicrystals are used as thin coating on conventional crystalline material in several technological applications. In this study, we present the STM observations of the initial growth of Al film deposited on $\mathrm{i}-\mathrm{Al}-\mathrm{Cu}-\mathrm{Fe}$ fivefold surface.

A similar system (Al deposited on i-AlPdMn fivefold surface) has been studied using low energy electron diffraction (LEED). It is found that the Al film of about $20 \AA$ thick consisting of $\mathrm{Al}$ nanocyrstals grows in the face-centered cubic structure in five different sets of domains [7]. STM measurements have great advantage to provide information of the film structure at atomic scale. Local information is of particular importance when substrate with structure as complex as quasicrystals are studied, where the inherent quasiperiodic surface structure can crucially affect the nucleation and growth of adlayers.

\section{Experimental Description}

The experiments were performed in an ultrahigh vacuum (UHV) chamber (base pressure $\sim 3.0 \times 10^{-11}$ Torr), equipped with an Omicron VTSTM as well as the instrumentation necessary for sample preparation and characterization. The i-AlCuFe substrate was cleaned by cycles of ion bombardment and subsequent annealing. The sample treatment produced the surface exhibiting fivefold LEED pattern and step-terrace structure 
in STM measurements. The high-resolution STM images accordingly revealed the ten-petalflower features on the atomic flat terraces [4].

Aluminum films were grown by physical vapor deposition. The Al evaporator consisted of an alumina tube $(4.77 \mathrm{~mm}$ o.d., $3.17 \mathrm{~mm}$ i.d.) into which a pure aluminum rod (3.07mm diameter) was inserted. The alumina tube was then fit tightly into a tungstenheating coil (seven turns of $0.1 \mathrm{~mm}$-diamter wire). To operate, the tungsten wire was resistively heated, in turn heating the alumina tube and the encased aluminum rod. During the deposition, the quasicrystalline substrate was hold at room temperature, and the background pressure remained below $6 \times 10^{-10}$ Torr.

To estimate the deposition flux, we deposited aluminum on $\mathrm{Al}$ (111) surface. The $\mathrm{Al}$ coverage, in terms of monolayer (ML), was then estimated by calculating the area fraction covered by Al islands in the images. Within the coverage regime studied in this work, the Al coverage is linear with dosing time. The Al coverage on the $\mathrm{i}-\mathrm{AlCuFe}$ fivefold surface was taken as the same as that on $\mathrm{Al}$ (111) surface under the same deposition conditions. Such an estimation is reasonable since the most favorable terminations for $\mathrm{i}-\mathrm{AlCuFe}$ fivefold surface is Al-rich, with atomic density close to that of a single plane of $\mathrm{Al}$ (111) [3].

\section{Experimental Results}

Fig. la represents a typical STM image obtained from the surface after low coverage of $\mathrm{Al}$ deposition. The white frame exhibits part of the image at a contrast that reveals the structure on the nascent i-AlCuFe fivefold surface dominated by flowers. The protrusions (light gray contrast) of about $1.0 \AA$ high are present on the surface before deposition [4]. 
The deposited $\mathrm{Al}$ atoms aggregate into pentagonal islands, which we call "starfish". The lineprofile (Fig. 1b) reveals that the starfish is about $5.1 \AA$ wide on the edge and about $2.3 \AA$ high. All the starfish are the same size and the same shape, although a few lack a "leg" (e.g. the circled one in Fig. 1a). Remarkably, they adopt the same orientation, not only within the same terrace, but also across different terraces. Furthermore, each of the five edges of the starfish (rotated by $72^{\circ}$ with respect to each other) finds itself parallel to one of the five principle directions on the substrate, where the principle directions are defined as the directions of the five sets of the parallel lines [4] that connect the centers of the flowers on the substrate. The dashed line crossing the two apparent flowers in Fig. 1a gives one ofsuch principle directions.

Once formed, the starfish does not grow laterally. With slightly increasing coverage, the density of starfish on the surface increases. Some of the adjacent starfish merge via shared leg. Nevertheless, the individual starfish remains intact (Fig. 2). As coverage increases, the surface gests bumpy, which worsen the resolution of the obtained STM images. We are not able to tell any specific structural features of the growing film (Fig. 3a), whereas further deposition produces three-dimensional islands, as shown in Fig $3 \mathrm{~b}$.

The formation of the starfish is insensitive to deposition flux over the investigated range of two orders of magnitude $\left(8 \times 10^{-5}\right.$ to $\left.7 \times 10^{-3} \mathrm{ML} / \mathrm{s}\right)$. Fig. $2 \mathrm{a}$ and $2 \mathrm{~b}$ show the similarity between two surfaces upon same coverage of deposition but at different flux.

These features suggest that the quasicrystalline substrate contain specific surface sites, which induce the nucleation of the pentagonal starfish preserving the fivefold symmetry of the substrate. The starfish possess certain stability, not willing to accept additional deposited $\mathrm{Al}$ atoms to grow laterally. 
The observations described up until now are true over most of the terraces on the surface. However, a few terraces accommodate strikingly different morphology upon Al deposition - the deposited $\mathrm{Al}$ atoms form fewer but larger, smoother islands (Fig. 4), with no apparent fivefold symmetry. These terraces tend to be bounded by steps of $2.4 \AA$ and 4.0 $\AA ̊$ high. Such "special" terraces again are observed at different flux.

\section{Discussions}

\subsection{Identification of the nucleation sites}

Relying on STM images only, it is hardly possible to identify the specific surface sites where the starfish nucleate. We resort to the available bulk model (similar to the one described in Ref. [8]) to achieve this goal. The $(x, y, z)$ coordinates in 3D space define the position of each atom. Taking fivefold axis as z-axis, all the atoms in the same atomic plane perpendicular to fivefold axis essentially have the same $\mathrm{z}$ coordinates. In the following discussions, we label atomic plane by the $\mathrm{z}$ coordinates of the atoms in that plane. Analysis described in the previous paper [4] confirms that the plane $(z=1.23 \AA)$ matches the STM images of clean fivefold surface of $\mathrm{i}-\mathrm{AlCuFe}$. We take this plane as the topmost layer exposed to the surface. Fig. 5a displays the atomic arrangements in an area of $10 \times 10 \mathrm{~nm}^{2}$ in this plane.

By considering the shape of the starfish, several types of pentagons outlined in Fig. $5 \mathrm{a}$ are taken as the candidates for the nucleation sites. The black pentagons can be easily ruled out since they are too small (edge length of $2.901 \AA$ ) to accommodate the starfish. The hatched and the gray colored pentagons have the same sizes with edge of $4.695 \AA$, close enough to the size of the starfish. However, the hatched pentagons are orientated in two 
different directions (rotated $\pi / 5$ with respect to each other), which would result the formation of the starfish in two equally probable orientations, whereas only one orientation appears in STM images. Same problem also applies to the black pentagons. Furthermore, the hatched pentagons possess asymmetric internal features, possibly leading to the formation of starfish with broken fivefold symmetry, which is clearly not observed in the experiments. Therefore, the hatched pentagons cannot be where the starfish nucleate. This leaves us the empty pentagons as the best candidates, all of which are marked gray in Fig. 5. They are all aligned in parallel in plane $(z=1.23 \AA)$. Such alignment holds as well for the empty pentagons in other atomic planes perpendicular to fivefold axis (see e.g., Fig. 7).

To check the vertical dimension of these empty pentagons, we probe several layers beneath this topmost layer. The atomic planes of $z=0.76,0$ and $-0.76 \AA$ follow the plane ( $z$ $=1.23 \AA$ ) in the bulk side, being $0.77,1.23$ and $1.99 \AA$ below the topmost layer respectively. We stack these planes together to reveal the vertical configurations around the empty pentagon sites. For better illustration, only a small region around one empty pentagon site is shown (Fig. 5b). Similar construction is found around other empty pentagons also. Clearly, the empty sites on plane $(z=1.23 \AA)$ remain empty as deep as $1.99 \AA$. Further analysis shows that the depth of the empty pentagons varies from 0.76 to $1.99 \AA$ if different planes are taken as topmost layer (Table 2).

As similar as for the starfish, each of the five edges of the empty pentagons finds itself parallel to one of the five principle directions (Fig. 5a). This geometric argument is also true for and only for the pentagons that are rotated by $\pi / 5$ (or multiples of $\pi / 5$ ) with respect to the empty one. Therefore, two possible orientations - either overlaying or rotated $\pi / 5$ with respect to the empty pentagons - are allowed for the deposited $\mathrm{Al}$ atoms to reside 
on the empty pentagons and as well agree with the observed starfish orientation with respect to the substrate. However, we will show below that the latter orientation is more favored.

\subsection{Nucleation model}

The identification of empty pentagonal surface sites as deep as $1.99 \AA$ leads naturally to the nucleation model as schematically illustrated in Fig. 6a-c. We postulate that a deposited $\mathrm{Al}$ atom diffuses across the surface until it drops into the hole at the center of the empty pentagons and gets trapped (Fig. 6b). This trapped central atom then helps to stablize five more $\mathrm{Al}$ atoms around its periphery to form starfish (Fig. 6c).

Based on STM measurement, the starfish exhibit a shallower central depression at maximum of $0.8 \AA$ below the starfish legs. This central depression is attributed to the deposited $\mathrm{Al}$ atom trapped in the central hole of empty pentagon sites, and embedded slightly within the substrate plane. This also explains why the hatched pentagons with filled asymmetric internal structure are not capable for the nucleation of the starfish - they lack the central hole that traps the deposited $\mathrm{Al}$ atom to initiate the nucleation.

Based on bulk model, plane $(z=2.75 \AA)$ is the one above the plane $(z=1.23 \AA)$. As shown in Fig. 7, five atoms in the upper layer form pentagons that are rotated $\pi / 5$ with respect to the empty pentagons in the lower layer. Therefore, it is reasonable for the deposited $\mathrm{Al}$ atoms to be stabilized at the five sites shown in Fig. 6c, since these sites are close to bulk lattice sites, i.e. sites that atoms would occupy if the entire upper layer were filled as it would be in the bulk structure. However, the lateral separation of the $\mathrm{Al}$ atoms in the starfish is larger than the separation of the true lattice sites. This can be justified by the 
fact that the central $\mathrm{Al}$ atom occupies a site that would remain vacant in the bulk structure; hence, 'pushes out' the surrounding five $\mathrm{Al}$ atoms.

\subsection{Analysis on other atomic planes}

It is tempting to look at other atomic planes since the planes in quasicrystalline structure are all different due to the lack of periodicity. Previous study [3] suggests that the closely-spaced atomic planes can be sorted in three recurring groups encompassing 3,5 , and 9 (or11) planes respectively and the bulk tends to terminate between different groups where the inter-planar spacings are large. The sets of 3,5 , and 9 planes give the step heights of $2.47,3.99$ and $6.47 \AA$. We apply the above analysis to the atomic planes within a depth of 3 $\mathrm{nm}$ in the bulk model and pick out all the atomic planes that have the chance to expose at the surface. Some of these atomic planes are highlighted in Table 1.

The plane ( $\mathrm{z}=1.23 \AA$ ) we have analyzed in section 4.1 is associated with stepheight of $3.99 \AA$. Other planes associated with the $3.99 \AA$-stepheight give the similar construction, although the density of the empty pentagons (surface nucleation sites for starfish), which are summarized in Table 2, varies from plane to plane.

Now we look at the atomic planes associated with $6.47 \AA$-stepheights. For instance, take the plane ( $\mathrm{z}=-19.68 \AA$ ) as the topmost layer and being exposed at the surface (Fig. 8). Only three complete flower-patterns (hatched circles) and some others with unfulfilled inner ring (gray circles) are found in this plane. Even taking the dashed circles into account, the plane seems not to contain enough flower-patterns to match the STM images obtained from the nascent substrate [4]. However, it is still not quite convincing to conclude that the plane is not the one probed by STM at this point. After all, we are not matching up the STM 
images with the atomic plane atom by atom. Many empty pentagons exist in the plane $(\mathrm{z}=-$ 19.68 ). Several examples are shown in Fig. 8. We notice that these empty pentagons are orientated in two directions rotated $\pi / 5$ with respect to each other (comparing the hatched and the gray pentagons), thus cannot be where the starfish nucleate for the reason discussed in section 4.1.

Surprisingly, taking the plane $(z=-20.15 \AA)$, which is $0.47 \AA$ below the previous one, as the topmost layer gives different story (Fig. 9a). The most notable features in this plane are the flower-patterns, consistent with the observed STM images from the nascent surfaces. The empty pentagons in this plane all adopt the same orientation and they remain empty as deep as $0.77 \AA$ until one atom occupies the center in plane $(z=-20.92 \AA)$ (see Fig. $9 b$ ). The previously proposed nucleation model is capable to fit the starfish with these empty pentagon sites. Apparently, the density of the nucleation sites in this plane is high (see Table 2), and some empty pentagons share vertex, which induces the starfish to merge via shared legs, as show in Fig. 2.

The idea - probing a layer below the predicted terminations in order to fit the experimental observations - holds true for the atomic planes associated with setpheight of both 6.47 and $2.47 \AA$. Such misfit is probably a indication of non-perfect bulk model. Work is still underway to do the similar analysis with more recent improved bulk model when it becomes available to us.

There are no empty pentagon sites in the latter case (see Table 2 ) within the investigated area of $10 \times 10 \mathrm{~nm}^{2}$. We also notice here that a couple of planes associated with $3.99 \AA$-stepheight contain no empty pentagons, thus not able to induce the formation of the starfish according to our nucleation model. These planes are speculated as the special 
terraces where the larger, smoother islands form upon Al deposition (see Fig. 4). It is consistent with the observation that the special terraces with smooth Al island formation tend to be boarded by $2.4 \AA$ and $4.0 \AA$ steps in STM images.

\subsection{The growth of film with increasing coverage}

On the terraces where the starfish form, the deposited $\mathrm{Al}$ atoms do not form entire psuedomorphic layer as coverage increases. Instead, they tend to grow three dimensionally (Fig. 3b). This is probably due to a combination of high interfacial energy (high strain) for an extended pseudomorphic aluminum layer on a quasicrystalline substrate, and the low surface energy of the substrate. The low apparent low surface energy of quasicrystals has long been noted [9].

\section{Conclusions}

In conclusion, the STM studies show that the Al forms pesudomorphic starfish on fivefold surface of icosahedral Al-Cu-Fe. We postulate that the formation of starfish is intrigued from a diffusing Al atom dropping into an empty site at the center of substrate pentagon and thereafter five additional $\mathrm{Al}$ adatoms filling the (near) lattice sties.

\section{Acknowledgements}

This work was supported by the Director, Office of Science, Office of Basic Energy Science, Materials Science Division of the U. S. Department of Energy, and by the EPSRC of the United Kingdom. 


\section{References}

1. C. Janot, Quasicrystals: A Primer, 2nd ed. (Oxford Univeristy Press, Oxford, 1994)

2. J. Ledieu, R. McGrath, R. D. Diehl, T. A. Lograsso, D. W. Delaney, Z. Papadopolos and G. Kasner, Surface Sci. Lett., 492 (2001) L729-L734

3. T. Cai, F. Shi, Z. Shen, M. Gierer, A. I. Goldman, M. J. Kramer, C. J. Jenks, T. A. Lograsso, D. W. Delaney, P. A. Thiel and M. A. Van Hove, Surface Science (2001) in press.

4. T. Cai, V. Foumée, T. Lograsso, A. R. Ross and P. A. Thiel, Phys. Rev. B: Rapid Communications (2001) submitted

5. M. Shimoda, T. J. Sato, A. P. Tsai and J. Q. Guo, Phys. Rev. B 62 (2000) 11288

6. M. Shimoda, J. Q. Guo, T. J. Sato and A. P. Tsai, Surf. Sci. 482-485(2) (2001) 784

7. B. Bolliger, V. E. Dmitrienko, M. Erbudak, R. Lüscher, H. -U. Nissen and A. R. Kortan, Phys. Rev. B 63 (2001) 052203

8. M. Boudard, M. de Boissieu, C. Janot, G. Heger, C. Beeli, H. -U. Nissen, H. Vincent, R. Ibberson, M. Audier and J. M. Dubois, J. Phys. Condens. Matter 4 (1992) 10149

9. Dubois, J. M. et al. Ann. Chim. Fr. 19 (1994) 3-25 


\section{Table and Figure Captions}

Table 1. Atomic composition, atomic density and inter-plane spacing from bulk model. $\mathrm{N}-\mathrm{Al}, \mathrm{N}-\mathrm{Cu}, \mathrm{N}-\mathrm{Fe}$ and Total: The amount of $\mathrm{Al}, \mathrm{Cu}, \mathrm{Fe}$ and total atoms in the area of the plane investigated. $Z(\AA)$ : the $z$ coordinates of the atoms in the plane.

Table 2. Summary of empty pentagon surface sites on different atomic planes

Figure 1. (a) STM image $\left(42 \times 42 \mathrm{~nm}^{2}\right)$ after $0.04 \mathrm{ML} \mathrm{Al}$ deposition at flux of $1.7 \times$ $10^{-3} \mathrm{ML} / \mathrm{s}$. Region bounded in white frame: different contrast applied to reveal the substrate structure. Dashed circle: starfish with one missing leg. Dashed line: one of the five principle directions on the substrate.

(b) Lineprofile along the solid line in (a) across two adjacent legs of starfish.

Figure 2. STM images $\left(30 \times 30 \mathrm{~nm}^{2}\right)$ after $0.1 \mathrm{ML}$ of Al deposition at flux of (a) 1.7 $\times 10^{-3} \mathrm{ML} / \mathrm{s}$ and (b) $6.7 \times 10^{-3} \mathrm{ML} / \mathrm{s}$, respectively.

Figure 3. (a) STM image $\left(100 \times 100 \mathrm{~nm}^{2}\right), 0.2 \mathrm{ML}$ of $\mathrm{Al}$ deposition

(b) STM image $\left(150 \times 150 \mathrm{~nm}^{2}\right), 1.0 \mathrm{ML}$ of Al deposition. The bottom panel: lineprofile along the line in the image.

Figure 4. STM image $\left(65 \times 65 \mathrm{~nm}^{2}\right), 0.2 \mathrm{ML}$ of Al deposition. Upper part of the image shows the special terrace that accommodates the growth of smooth $\mathrm{Al}$ islands, as revealed from the lineprofile.

Figure 5. (a) $10 \times 10 \mathrm{~nm}^{2}$ truncation of plane $(\mathrm{z}=1.23 \AA)$ from bulk model. Filled markers: Al atoms. Unfilled markers: Fe atoms. Black pentagons: pentagons with edge of 2.901 A. Hatched pentagons: pentagons filled asymmetrically with atoms. Both black and hatched pentagons have two different orientations. Light gray pentagons: all the empty pentagons in the plane. Circles: outlines of several examples of flower-patterns on the 
substrate. Crossing lines: five principle directions lining up the centers of flowers on the substrate.

(b) Stacks of several successive layers. Black solid circle, empty circle, cross, and solid gray markers label the atomic positions in plane $z=1.23,0.76,0$ and $-0.76 \AA$, respectively.

Figure 6. Schematic of the formation of starfish. Solid circles: atoms on the substrate. Hatched circles: deposited Al atoms. (a) empty pentagon, the nucleation sites on the substrate. (b) one deposited $\mathrm{Al}$ atom drops into the hole at the center of the empty pentagon and gets trapped. (c) Five more deposited $\mathrm{Al}$ atoms are stabilized at the periphery of the empty pentagon and form the starfish.

Figure 7. Stacks of two layers from bulk model. Solid circles and empty circles label the atomic positions in the plane $(z=1.23 \AA)$ and plane $(z=2.75 \AA)$ respectively.

Figure 8. 10x10nm ${ }^{2}$ truncation of plane $(\mathrm{z}=-19.68 \AA)$ from bulk model. Filled markers: Al atoms. Unfilled markers: $\mathrm{Fe}$ atoms. Hatched circles: complete flower-patterns. Gray circles: flower-patterns with unfilled inner-ring. Hatched and gray pentagons: examples of empty pentagons in the plane. They are rotated by $\pi / 5$ with respect to each other.

Figure 9. (a) $10 \times 10 \mathrm{~nm}^{2}$ truncation of plane $(z=-20.15 \AA)$ from bulk model. Filled markers: $\mathrm{Al}$ atoms. Cross markers: $\mathrm{Cu}$ atoms. Unfilled markers: Fe atoms. Gray circles: flower-patterns on the substrate. Gray pentagons: empty pentagon surface sites.

(b) Stacks of several successive layers. Black solid circle, cross, and empty markers label the atomic positions in plane $z=-20.15,-20.62$ and $-20.92 \AA$. 
Table 1

\begin{tabular}{|c|c|c|c|c|c|c|}
\hline $\mathrm{N}-\mathrm{Al}$ & $\mathrm{N}-\mathrm{Cu}$ & $\mathrm{N}-\mathrm{Fe}$ & Total & $Z(\AA)$ & Spacing & Stepheight $(\AA)$ \\
\hline 570 & 0 & 276 & 846 & 2.75 & & \\
\hline 156 & 㴧 & 242 & 1602 & ${ }_{123}$ & 墕52 & \\
\hline 382 & 539 & 0 & 921 & 0.76 & 0.47 & \\
\hline 0 & 133 & 0 & 133 & 0 & 0.76 & 3.99 \\
\hline 364 & 562 & 0 & 926 & -0.77 & 0.76 & \\
\hline 1380 & 0 & 220 & 1600 & -1.24 & 0.47 & \\
\hline 689 & 0 & 272 & 864 & 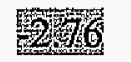 & 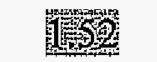 & \\
\hline 579 & 809 & 201 & 1589 & -3.24 & 0.48 & \\
\hline 187 & 0 & 0 & 187 & -3.71 & 0.47 & \\
\hline 0 & 13 & 0 & 13 & -4 & 0.29 & \\
\hline 113 & 0 & 0 & 113 & -4.76 & 0.76 & \\
\hline 1145 & 0 & 482 & 1627 & -5.23 & 0.47 & 6.47 \\
\hline 476 & 161 & 0 & 637 & -5.7 & 0.47 & \\
\hline 0 & 155 & 0 & 155 & -6.47 & 0.77 & \\
\hline 13 & 0 & 0 & 13 & -6.76 & 0.29 & \\
\hline 537 & 652 & 76 & 1265 & -7.23 & 0.47 & \\
\hline 1186 & 0 & 42 & 1228 & -7.7 & 0.47 & \\
\hline 136 & 㥜 & 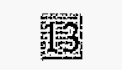 & 1389 & 1528 & 13 & \\
\hline 316 & 776 & 2 & 1094 & -9.7 & 0.47 & \\
\hline 0 & 212 & 0 & 212 & -10.46 & 0.76 & 3.99 \\
\hline 548 & 289 & 0 & 837 & -11.22 & 0.76 & \\
\hline 1169 & 0 & 422 & 1591 & -11.69 & 0.47 & \\
\hline 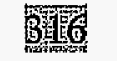 & 算 & 82 & 398 & 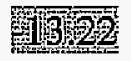 & 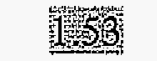 & \\
\hline 462 & 948 & 187 & 1597 & -13.69 & 0.47 & \\
\hline 373 & 0 & 203 & 576 & -14.16 & 0.47 & 2.47 \\
\hline 1218 & 䈍 & 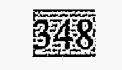 & 166 & 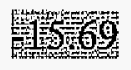 & Is & \\
\hline 520 & 327 & 0 & 847 & -16.16 & 0.47 & \\
\hline 0 & 226 & 0 & 226 & -16.92 & 0.76 & 3.99 \\
\hline 217 & 741 & 1 & 959 & -17.68 & 0.76 & \\
\hline 1346 & 0 & 54 & 1400 & -18.16 & 0.48 & \\
\hline 8 & 䍖 & 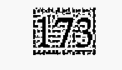 & 1043 & 1968 & 1 & \\
\hline 613 & 578 & 118 & 1309 & -20.15 & 0.47 & \\
\hline 50 & 0 & 0 & 50 & -20.62 & 0.47 & \\
\hline 0 & 87 & 0 & 87 & -20.92 & 0.3 & \\
\hline 371 & 83 & 0 & 454 & -21.68 & 0.76 & \\
\hline 1089 & 0 & 421 & 1510 & -22.15 & 0.47 & 6.46 \\
\hline 234 & 30 & 0 & 264 & -22.62 & 0.47 & \\
\hline 0 & 50 & 0 & 50 & -23.38 & 0.76 & \\
\hline 90 & 0 & 0 & 90 & -23.67 & 0.29 & \\
\hline 566 & 604 & 139 & 1309 & -24.15 & 0.48 & \\
\hline
\end{tabular}


Table 2

\begin{tabular}{|c|c|c|c|}
\hline Stepheight ( $\AA$ ) & Plane (z) & $\begin{array}{l}\text { Number of empty } \\
\text { pentagon sites in an } \\
\text { area of } 10 \times 10 \mathbf{n m}^{2}\end{array}$ & $\begin{array}{l}\text { Depth of empty } \\
\text { pentagon sites }(\AA)\end{array}$ \\
\hline \multirow{5}{*}{3.99} & 18.15 & 16 & 1.99 \\
\hline & 11.69 & 0 & \\
\hline & 1.23 & 6 & 1.99 \\
\hline & -9.23 & 22 & 1.22 \\
\hline & -15.68 & 0 & \\
\hline \multirow{4}{*}{6.46} & $24.14 *$ & 25 & 0.76 \\
\hline & $7.22 *$ & 71 & 0.76 \\
\hline & $-3.24 *$ & 7 & 2.00 \\
\hline & $-20.15^{*}$ & 45 & 0.76 \\
\hline \multirow[t]{2}{*}{2.47} & 13.68 * & 0 & \\
\hline & $-13.69 *$ & 0 & \\
\hline
\end{tabular}

- These atomic planes are $0.47 \AA$ below than the ones labeled in Table 1 as the most favorable terminations. 

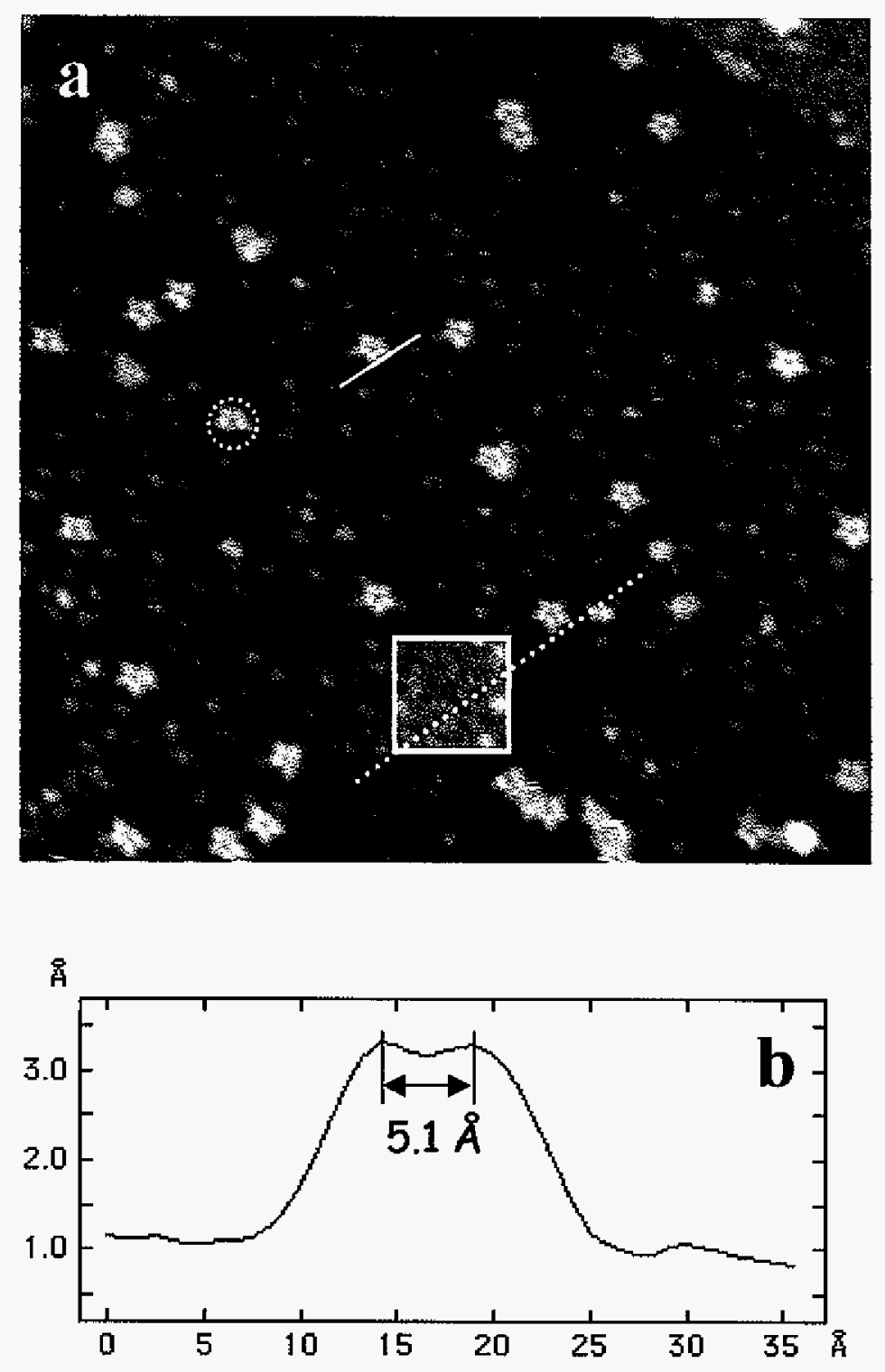

Figure 1 

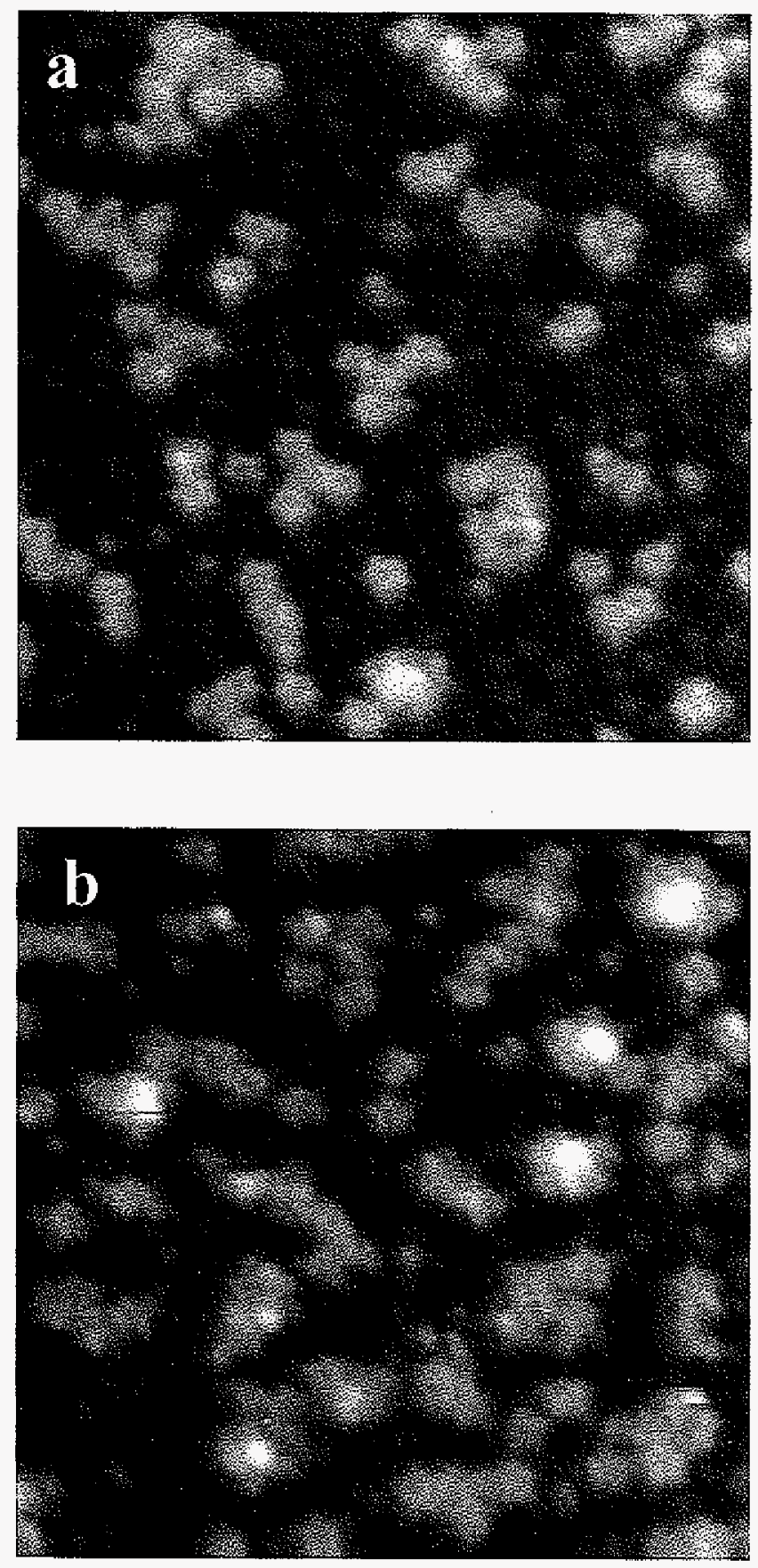

Figure 2 

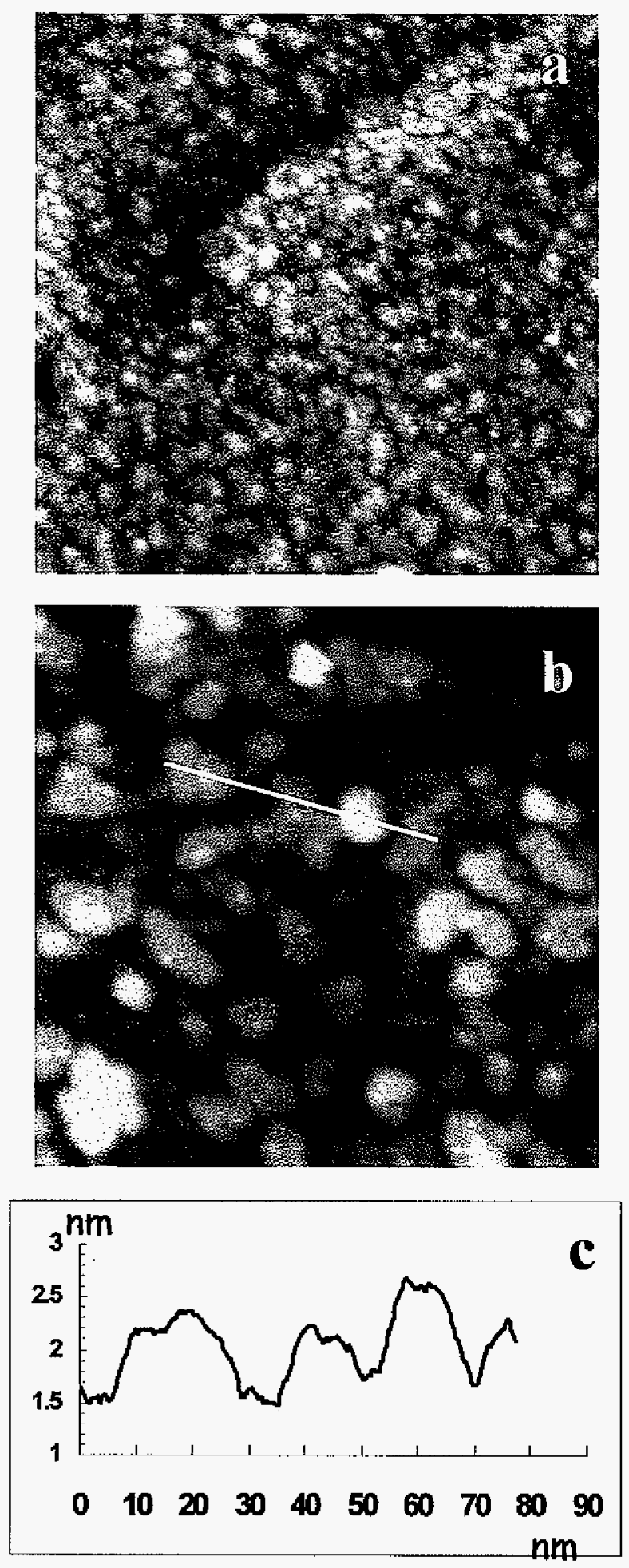

Figure 3 


$$
\text { ฤ ว.กชิเม }
$$
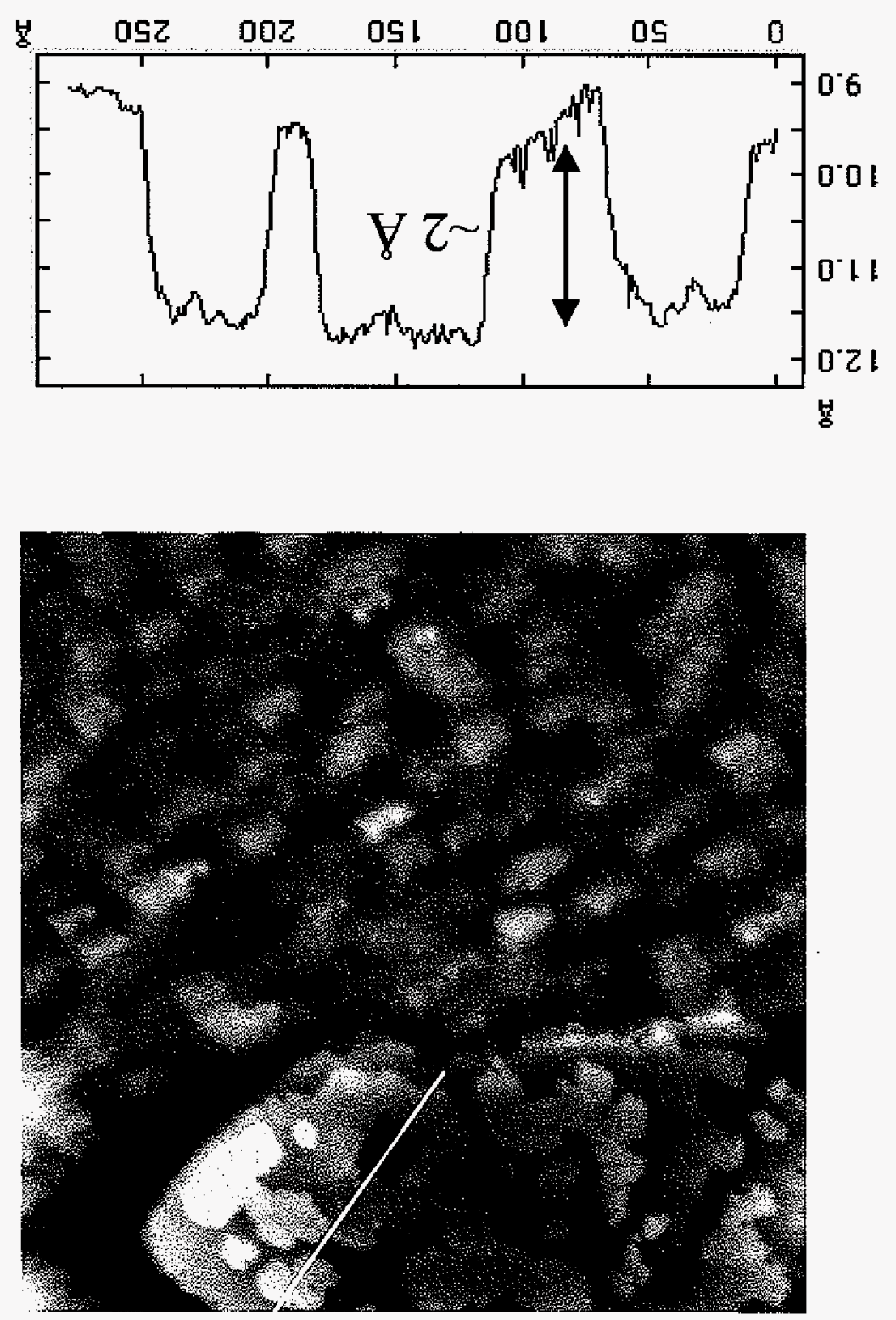

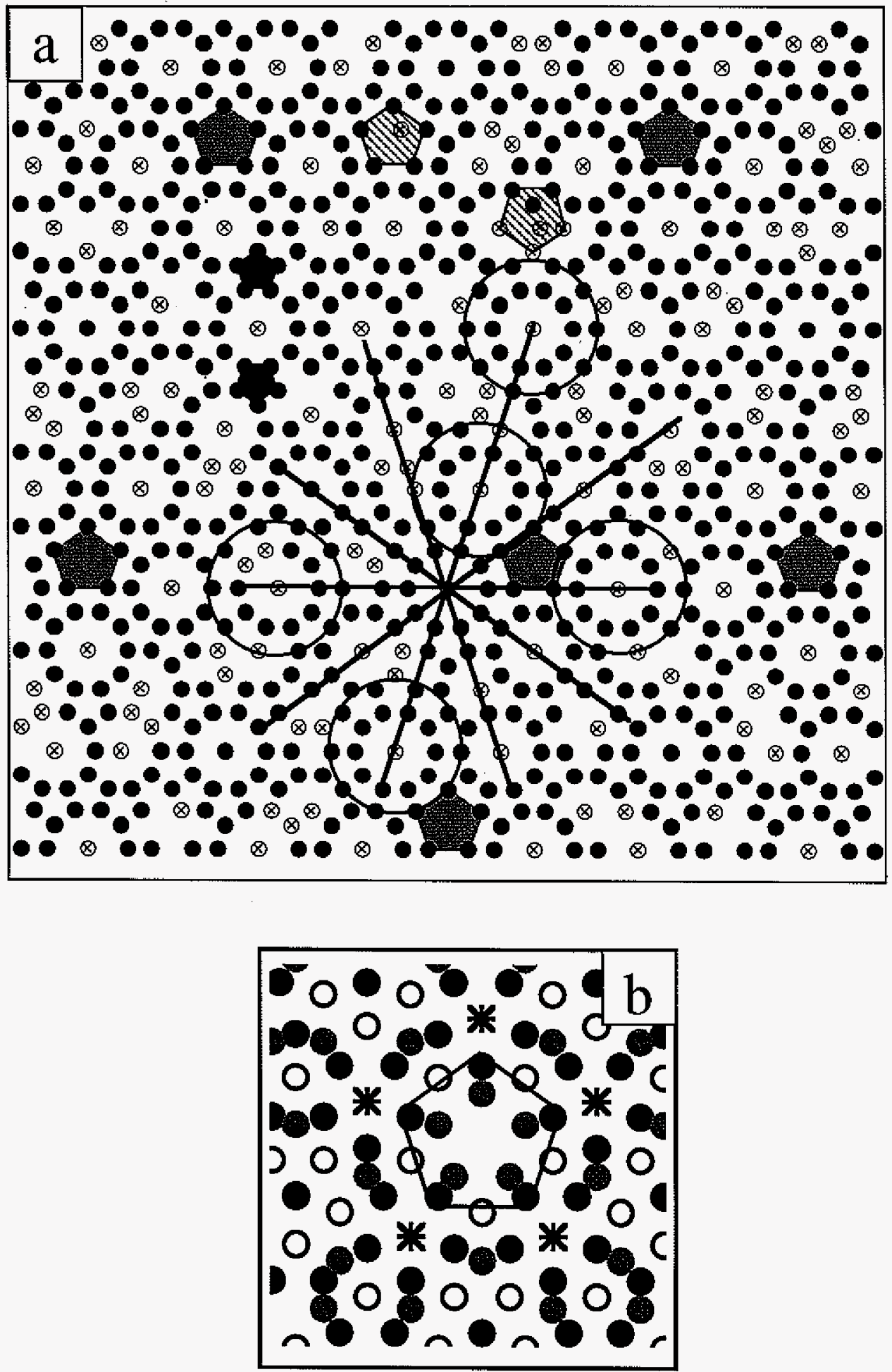

Figure 5 


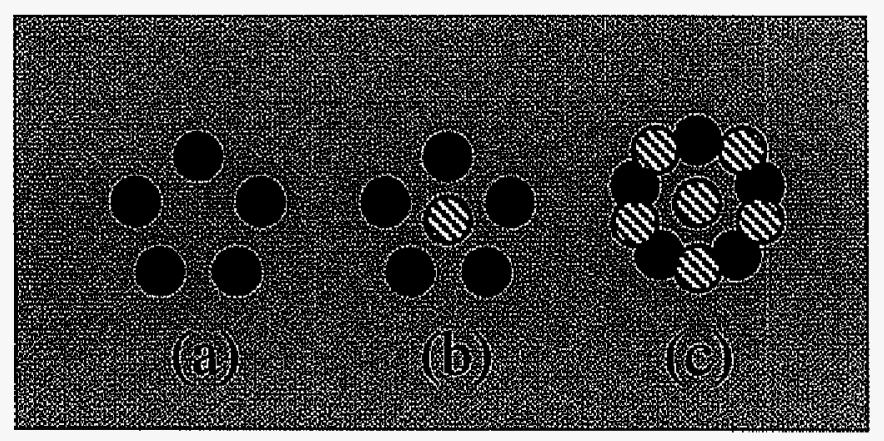

Figure 6 


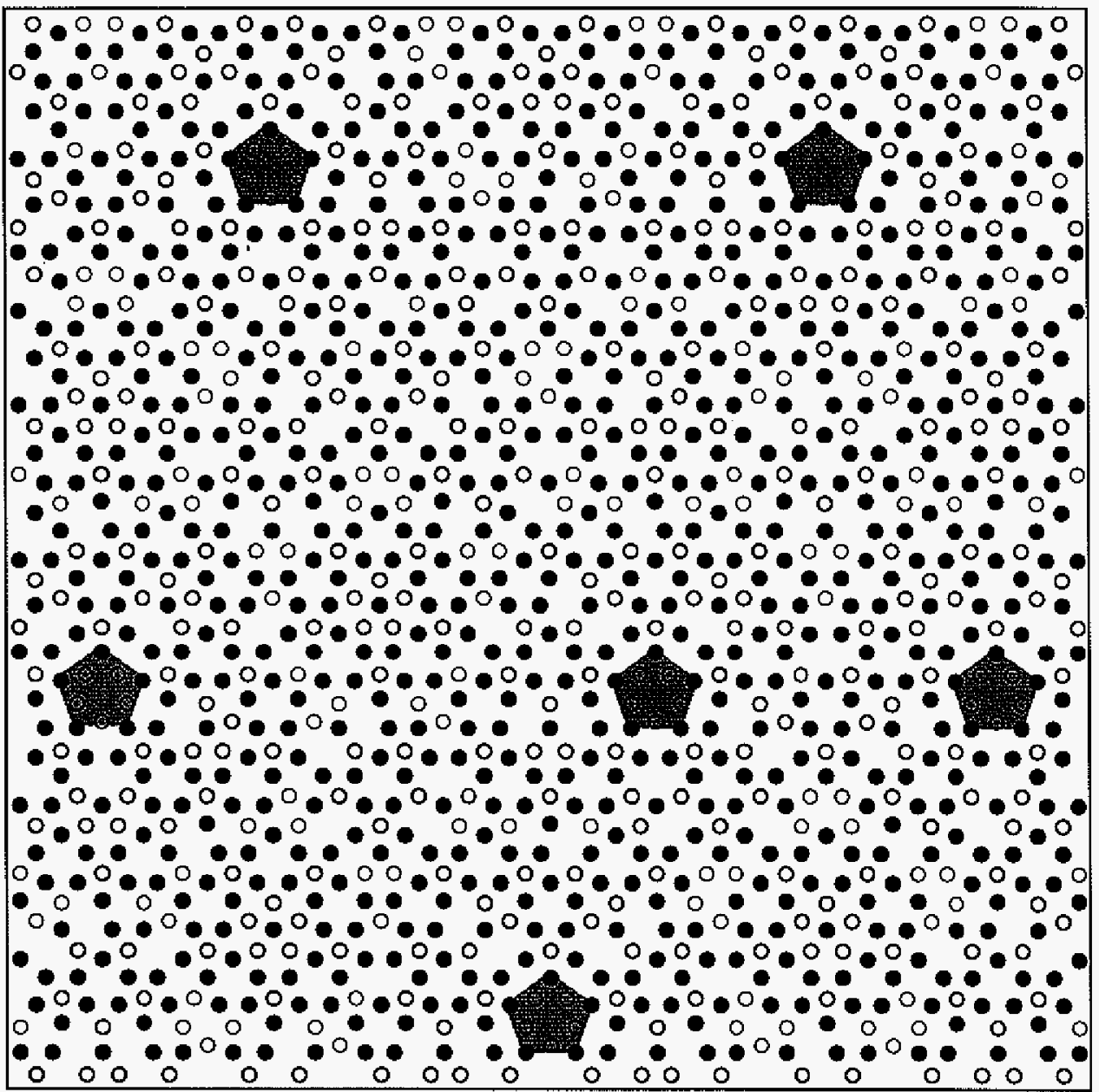

Figure 7 


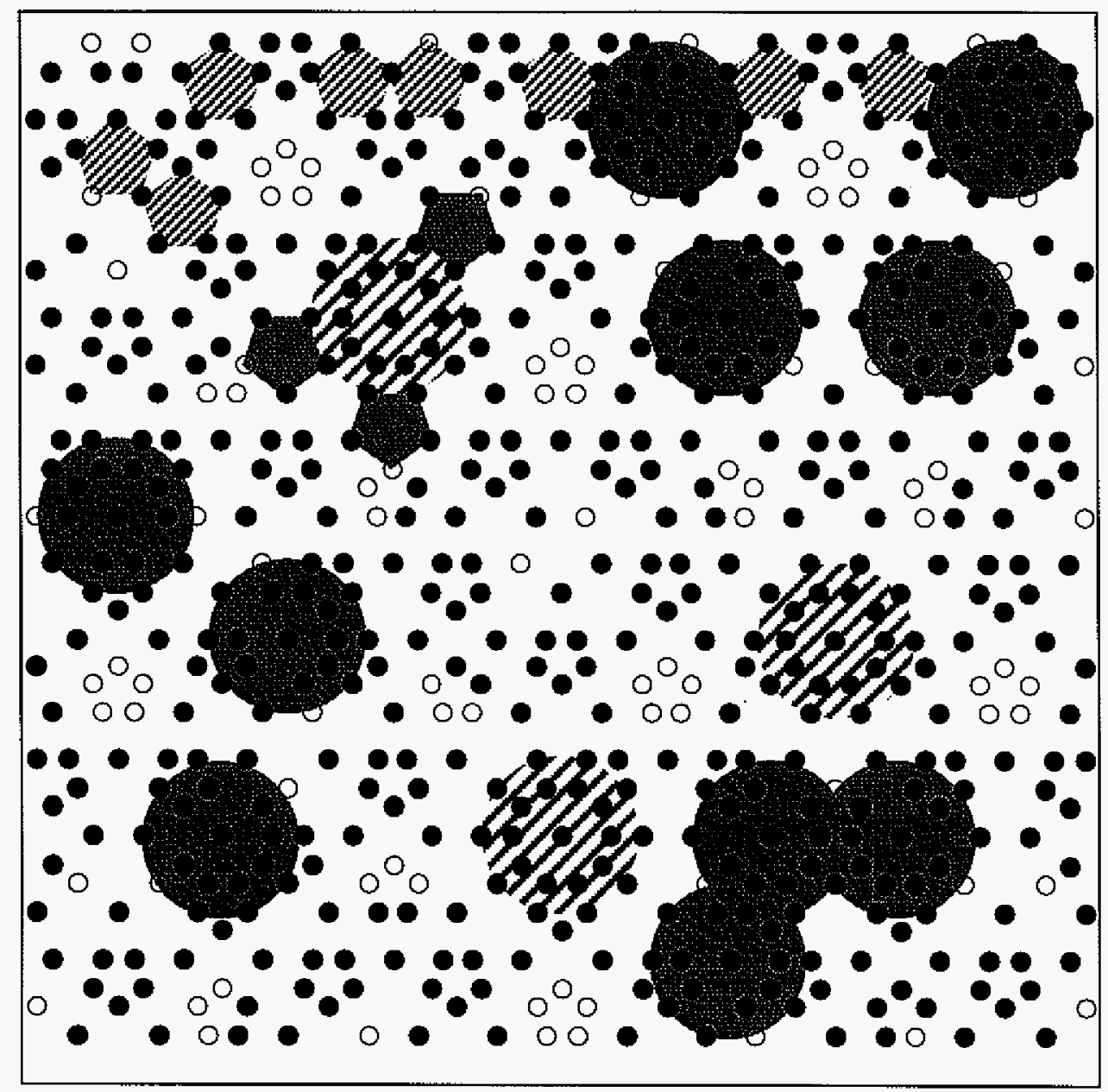

Figure 8 

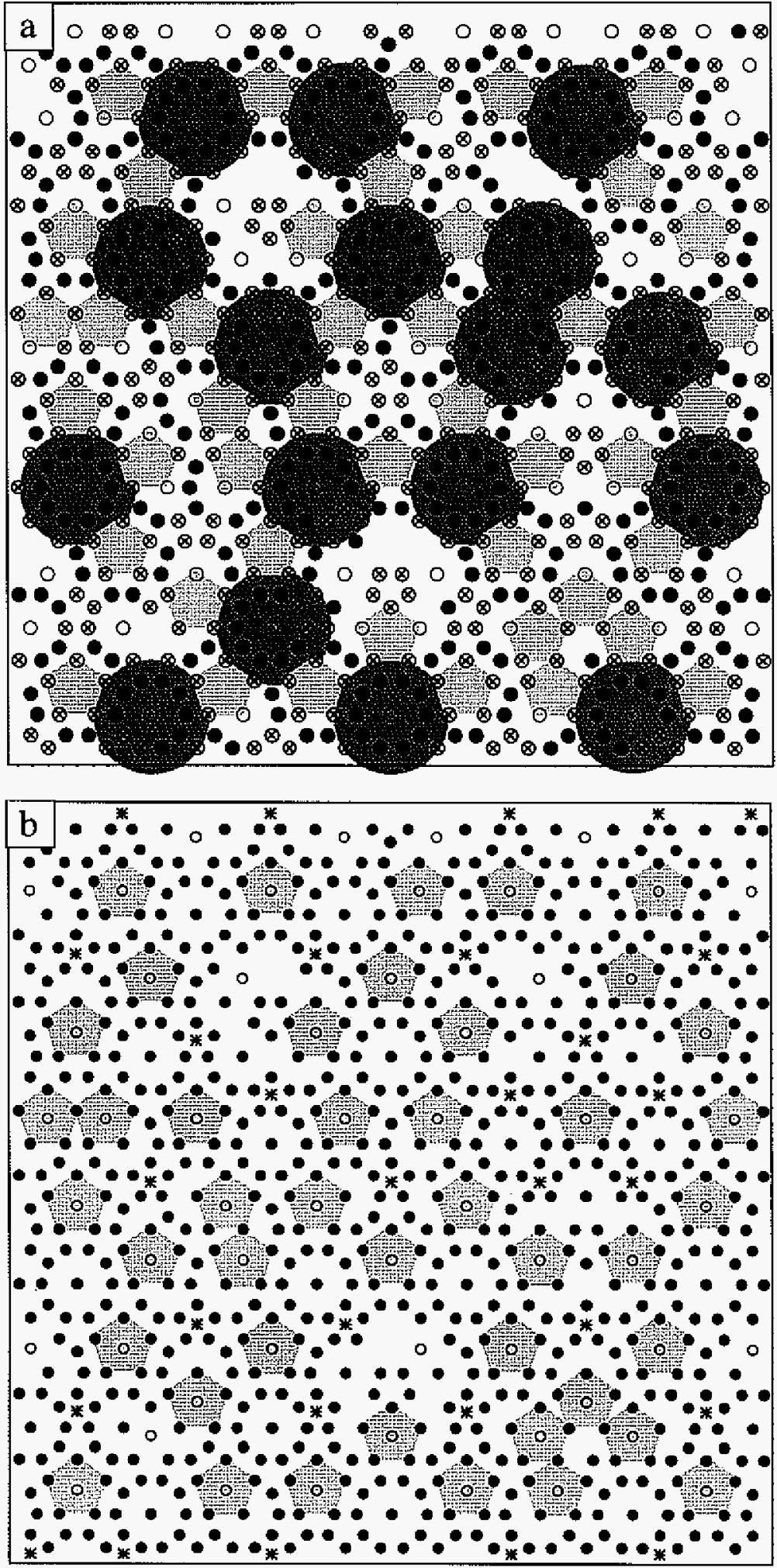

Figure 9 
APPENDIX D. STM DATABASE

Table 1. STM database of experiments on i-AIPdMn 3fold surface

\begin{tabular}{|c|c|c|c|c|}
\hline $\begin{array}{c}\text { File } \\
\text { Directory }^{I}\end{array}$ & $\begin{array}{c}\text { File } \\
\text { Name }^{2}\end{array}$ & $\begin{array}{c}\text { Sample Treatment } \\
\text { Annealing temp., } \\
\text { Annealing time }\end{array}$ & Image Description & $\begin{array}{l}\text { Images used } \\
\text { in the paper }\end{array}$ \\
\hline QC3f & $09 \times \times 98$ & \multicolumn{3}{|c|}{$\begin{array}{l}\text { surface was rough with huge bumps, } \\
\text { not able to get decent images }\end{array}$} \\
\hline \multirow{5}{*}{$\mathrm{QC} 3 \mathrm{f} 2$} & 101298 & $800 \mathrm{~K}, 2$ hours & clusters on terraces & m9-Fig. 2(b) \\
\hline & 101398 & $800 \mathrm{~K}, 4$ hours & clusters on terraces & m3-Fig. 2(a) \\
\hline & 101498 & $850 \mathrm{~K}, 2$ hours & no improvement & \\
\hline & 101698 & $900 \mathrm{~K}, 0.75$ hour & $\begin{array}{l}\text { terraces with straight and } \\
\text { kinked step edges, } \\
\text { triangular shaped terraces }\end{array}$ & $\begin{array}{l}\text { m5 - Fig. 4(a) } \\
\text { m22-Fig. } 4(\mathrm{~b}) \\
\text { m46-Fig. } 4(\mathrm{c})\end{array}$ \\
\hline & 101998 & $900 \mathrm{~K}, 2$ hours & $\begin{array}{l}\text { similar as on } 101698 \text {, but } \\
\text { images get worse }\end{array}$ & \\
\hline \multirow{5}{*}{ QC3f3 } & 111098 & $800 \mathrm{~K}, 1$ hour & $\begin{array}{l}\text { rough surface, } \\
\text { bumps } \sim 10 \mathrm{~nm}\end{array}$ & m7-Fig. 1 (b) \\
\hline & 111198 & $850 \mathrm{~K}, 1$ hour & clusters on terraces & \\
\hline & 111298 & $900 \mathrm{~K}, 0.5$ hour & $\begin{array}{l}\text { large amount of images of } \\
\text { clusters, nice terraces }\end{array}$ & m69-Fig. 3 (a) \\
\hline & 111698 & $900 \mathrm{~K}, 0.75$ hour & terraces & \\
\hline & 111998 & $900 \mathrm{~K}, 1.5$ hour & triangle terraces & \\
\hline \multirow{4}{*}{ QC3f4 } & 120498 & $850 \mathrm{~K}, 1$ hour & clusters on terraces & m11 - Fig. 1 (a) \\
\hline & 120898 & $850 \mathrm{~K}, 2$ hours & clusters on terraces & \\
\hline & 120998 & $875 \mathrm{~K}, 2$ hours & $\begin{array}{l}\text { nice terraces, some fine } \\
\text { structures, small triangular } \\
\text { pits }\end{array}$ & $\mathrm{m} 29-\mathrm{Fig} .3(\mathrm{~b})$ \\
\hline & 121298 & $900 \mathrm{~K}, 0.5$ hour & $\begin{array}{l}\text { nice large terraces, } \\
\text { tried oxidation experiments } \\
\text { but without success } \\
\text { (m40-m57) }\end{array}$ & \\
\hline
\end{tabular}

${ }^{1}$ Files were created on RT-STM Unix system (Room 224/225 Spedding)

Path: home/zhouxin/data/sample/

${ }^{2}$ Scheme to name the files

/QC3f

i-AIPdMn 3fold surface

Sample L.D.: ARR-1-26-1 (DWD-2-83-1)

$-1(2,3,4)-$

After sample was exposed to the air and was again cleaned via cycles of sputtering and annealing, the file would be saved in a different directory.

mmddyy the date when the file was created and saved.

${ }^{3}$ Images are used in paper "Structural aspects of the three-fold surface of icosahedral Al-Pd-Mn" (appearing in volume 461, issue I-3 of Surface Science on page L521-L527, 2000). 
Table 2. STM database of experiments on i-AlCuFe 5fold surface - Stepheight measurement

\begin{tabular}{|c|c|c|c|}
\hline File Name $^{1}$ & $\begin{array}{l}\text { Sample Treatment } \\
\text { Annealing temp., } \\
\text { Annealing time }\end{array}$ & Image Description $^{2}$ & $\begin{array}{c}\text { Images used in the } \\
\text { paper }^{3}\end{array}$ \\
\hline 020399 & $800 \mathrm{~K}$, 2hours & rough surface & \\
\hline 020899 & $825 \mathrm{~K}, 2$ hours & $\begin{array}{l}\text { terraces, part of the surface shows } \\
\text { clusters }\end{array}$ & m4-Fig. 1(c) \\
\hline 021299 & $850 \mathrm{~K}$, thour & terraces, screw dislocation & \\
\hline 021599 & $850 \mathrm{~K}, 2$ hours & $\begin{array}{l}\text { terraces, screw dislocations, } \\
\text { pentagon pits }\end{array}$ & \\
\hline 021899 & $850 \mathrm{~K}$, 2hours & pentagon pits & \\
\hline $\begin{array}{l}022399 \\
022699 \\
030499\end{array}$ & 875K, 1-2hours & terraces & \\
\hline $\begin{array}{l}030999 \\
031599 \\
031899 \\
\end{array}$ & \multicolumn{3}{|c|}{$\begin{array}{l}\text { Studies on } A g(100) \text { sample, measured stepheight }=2.4 \pm 0.2 A \\
\text { used to calibrate the stepheight measurement on quasicrystals. }\end{array}$} \\
\hline \multicolumn{4}{|c|}{ Change the STM scanner in early April, 1999} \\
\hline 042199 & $800 \mathrm{k}$, 1hour & rough surface & \\
\hline 042399 & $825 \mathrm{k}$, 1hour & terraces with screw dislocation & m9-Fig. l(b) \\
\hline 042699 & $825 \mathrm{k}, 1.5$ hour & clustered surfaces & mll - Fig. 1(a) \\
\hline $\begin{array}{l}042999 \\
050399 \\
\end{array}$ & $825 \mathrm{k}, 2 \mathrm{hours}$ & terraces, clusters & \\
\hline 051099 & $825 \mathrm{k}, 2 \mathrm{hours}$ & $\begin{array}{l}\text { nice terraces with screw disloactions } \\
\text { m17: showing fine structure on the } \\
\text { terraces }\end{array}$ & $\begin{array}{l}\text { m18-Fig. } 1(\mathrm{~d}) \\
\text { m19-Fig. } 1(\mathrm{e}) \\
\text { m26-Fig. I(f) }\end{array}$ \\
\hline \multicolumn{4}{|c|}{ Broke the vacuum and made some fixing in the chamber } \\
\hline 061699 & $825 \mathrm{k}, 2$ hours & clusters & \\
\hline 062199 & $850 \mathrm{~K}, 1.5$ hour & clusters on terraces & \\
\hline $\begin{array}{l}062399 \\
062899 \\
\end{array}$ & $850 \mathrm{~K}, 2$ hours & terraces, screw dislocations & \\
\hline 062599 & $850 \mathrm{~K}$, 2hours & $\begin{array}{l}\mathrm{m} 4, \mathrm{~m} 5 \text { :fine structure on the terrace, } \\
\text { ring-features! }\end{array}$ & \\
\hline 063099 & $875 \mathrm{~K}, 1.5$ hours & terraces & \\
\hline 070999 & $850 \mathrm{~K}$, 2hours & $\mathrm{ml}$ :nice screw dislocations & \\
\hline $\begin{array}{l}051799 \\
090299\end{array}$ & \multicolumn{3}{|c|}{ Studies on Ag(100) sample, measured stepheight $=2.8 \pm 0.2 A$} \\
\hline
\end{tabular}

${ }^{1}$ Files were created on RT-STM Unix system (Room 224/225 Spedding)

Path: home/zhouxin/data/sample/AlCuFe5f-mmddyy (mmddyy: the date when the file were saved).

Sample: i-AlCuFe 5fold surface. $\quad$ Sample I.D.: ARR-1-63, ARR-1-61

${ }^{2}$ Statistics shows that there are 3 screw dislocations in an area of $200 \times 200 \mathrm{~nm}^{2}$ on average.

${ }^{3}$ Images are used in paper "Structural aspects of the fivefold quasicrystalline AI-Cu-Fe surface from STM and dynamical LEED studies" (in press by Surface Science). 
Table 3. STM database of experiments on i-AlCuFe 5fold surface - Atomic scale structure and Al deposition experiments

\begin{tabular}{|c|c|c|c|c|}
\hline $\begin{array}{c}\text { File } \\
\text { Name }\end{array}$ & $\begin{array}{l}\text { Sample Treatment } \\
\text { (Annealing temp. } \\
\text { Annealing Time) }\end{array}$ & $\begin{array}{c}\text { Al deposition } \\
\text { flux at } \\
1.6 \times 10^{-3} \mathrm{ML} / \mathrm{s}\end{array}$ & Image Description & $\begin{array}{l}\text { Image Used } \\
\text { in the paper }{ }^{1,2,3,4}\end{array}$ \\
\hline 102700 & $700 \mathrm{~K}, 1$ hour & \multirow{7}{*}{ None } & Clusters on terraces & \\
\hline 103100 & $750 \mathrm{~K}$, lhour & & $\begin{array}{l}\text { Better terraces, but not fully } \\
\text { free from clusters }\end{array}$ & \\
\hline 110700 & $800 \mathrm{~K}, 1$ hour & & $\begin{array}{l}\text { Start to get fine structure on } \\
\text { the terraces }\end{array}$ & \\
\hline 110800 & $825 \mathrm{~K}, 1.5$ hour & & $\begin{array}{l}\text { Fruitful Day! Flower-patterns } \\
\text { on the terraces }\end{array}$ & $\begin{array}{l}\mathrm{m} 13-\text { Fig. } 1 \mathrm{~h}^{1} \\
\text { m33 - Fig. } 1 \mathrm{~g}^{1} \\
\text { and Fig. } 2 \text { FFT } \\
\text { m14 - Fig. } 2 \mathrm{a}^{2} \\
\text { m35 - Fig. } 2 \mathrm{~b}^{2}\end{array}$ \\
\hline 111400 & $825 \mathrm{~K}, 1.5$ hour & & $\begin{array}{l}\text { Flower-patters, large } \\
\text { pentagon pits on the terraces }\end{array}$ & \\
\hline 120400 & $825 \mathrm{~K}, 2$ hours & & Bad surface & \\
\hline 120600 & $875 \mathrm{~K}$, 2hours & & $\begin{array}{l}\text { m13: got back the flower- } \\
\text { patterns }\end{array}$ & \\
\hline 120700 & \multirow{7}{*}{$825 \mathrm{~K}, 2$ hours } & $1 \mathrm{ML}$ & $\begin{array}{l}\text { Three-dimensional growth of } \\
\text { Al films }\end{array}$ & $m 2-$ Fig. $3 b^{4}$ \\
\hline 121400 & & $0.5 \mathrm{ML}$ & Rough, clustered structure & \\
\hline 121800 & & $0.1 \mathrm{ML}$ & $\begin{array}{l}\text { "starfish" merge via shared } \\
\text { legs }\end{array}$ & m23-Fig. $2 a^{4}$ \\
\hline 122000 & & $0.04 \mathrm{ML}$ & Cool "starfish" & $\begin{array}{l}\text { m5-Fig. } 1^{3} \\
\text { and Fig. } \mathrm{Ia}^{4}\end{array}$ \\
\hline 010200 & & $0.05 \mathrm{ML}$ & \multirow{3}{*}{$\begin{array}{l}\text { Starfish, } \\
\text { but not well resovled }\end{array}$} & \\
\hline 010300 & & $0.02 \mathrm{ML}$ & & \\
\hline 010900 & & $0.03 \mathrm{ML}$ & & \\
\hline
\end{tabular}

'Files were created on VT-STM Window NT system (Room 224/225 Spedding)

Path: C:lOmicronldatalsamplelAlCuFe5f

${ }^{1}$ Images used in paper "Structural aspects of the fivefold quasicrystalline Al-Cu-Fe surface from STM and dynamical LEED studies" (in press by Surface Science).

${ }^{2}$ Images used in paper "An STM study of the atomic structure of the icosahedral Al-Cu-Fe fivefold surface" (submitted to Physical Review B, Rapid Communication)

${ }^{3}$ Images used in paper "Pseudomorphic starfish: arrangement of extrinsic metal atoms on a quasicrystalline substrate" (submitted to Nature).

${ }^{4}$ Images are used in Appendix C. 
Table 4. STM database of experiments on i-AlCuFe 5 fold surface

- Al deposition experiments (continued)

\begin{tabular}{|c|c|c|c|c|c|}
\hline \multirow{2}{*}{\multicolumn{2}{|c|}{ File Name ${ }^{1}$}} & \multicolumn{2}{|c|}{ Al deposition } & \multirow[b]{2}{*}{ Image Description } & \multirow[b]{2}{*}{$\begin{array}{l}\text { Image Used in } \\
\text { the paper }\end{array}$} \\
\hline & & $\begin{array}{c}\text { Flux } \\
(\mathrm{ML} / \mathrm{S})\end{array}$ & $\begin{array}{l}\text { Coverage } \\
\text { (ML) }\end{array}$ & & \\
\hline \multirow{2}{*}{\multicolumn{2}{|c|}{$\begin{array}{ll}062301 \\
062501\end{array}$}} & \multirow{2}{*}{\multicolumn{2}{|c|}{ None }} & Flower-patterns & \\
\hline & & & & $\begin{array}{l}\text { Flower-patterns, surface gets } \\
\text { dirty after Al deposition }\end{array}$ & \\
\hline \multirow{5}{*}{072401} & $\mathrm{ml-m16}$ & \multicolumn{2}{|c|}{ None } & Flower-patterns & \\
\hline & $\mathrm{m} 17-\mathrm{m} 31$ & \multirow{4}{*}{$1.7 \times 10^{-3}$} & 0.04 & Starfish not well resolved & \\
\hline & $\mathrm{m} 32-\mathrm{m} 39$ & & & $\begin{array}{l}\text { Gevengighth } \\
\text { apparent change }\end{array}$ & \\
\hline & $\mathrm{m} 40-\mathrm{m} 44$ & & 0.10 & Not well resolved structure & \\
\hline & $\mathrm{m} 45-\mathrm{m} 46$ & & 0.20 & Rough, bumpy surface & \\
\hline \multirow[t]{2}{*}{080101} & m1-m3 & \multicolumn{2}{|c|}{ None } & Flower-patterns & \\
\hline & $\mathrm{m} 4-\mathrm{m} 9$ & $1.7 \times 10^{-3}$ & 0.08 & & \\
\hline \multicolumn{2}{|c|}{091201} & \multirow{2}{*}{\multicolumn{2}{|c|}{ None }} & Clean surface & \\
\hline \multicolumn{2}{|c|}{091701} & & & 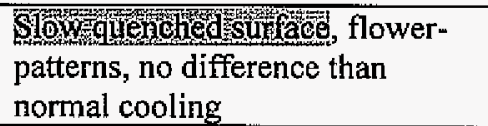 & \\
\hline \multirow{6}{*}{091901} & $\mathrm{~m} 1-\mathrm{m} 4$ & \multicolumn{2}{|c|}{ None } & & \\
\hline & m5-m27 & \multirow{5}{*}{$1.7 \times 10^{-3}$} & 0.1 & starfish & \multirow{5}{*}{ m40-Fig. 4} \\
\hline & $\mathrm{m} 28-\mathrm{m} 47$ & & 0.2 & $\begin{array}{l}\text { Special terraces with different Al } \\
\text { growth }\end{array}$ & \\
\hline & $\mathrm{m} 48-\mathrm{m} 55$ & & 0.4 & m54: special terraces & \\
\hline & $\mathrm{m} 56-\mathrm{m} 58$ & & 0.8 & Rough surface & \\
\hline & m59-m64 & & 2.0 & & \\
\hline \multicolumn{2}{|c|}{092601} & \multicolumn{2}{|c|}{ None } & $\begin{array}{l}\text { m11-m18, special terraces on } \\
\text { clean surface? }\end{array}$ & \\
\hline 092701 & $\mathrm{~m} 7-\mathrm{m} 31$ & $1.7 \times 10^{-3}$ & 0.15 & $\mathrm{~m} 12$ : different Al growth & \\
\hline \multicolumn{2}{|c|}{092801} & $8.5 \times 10^{-5}$ & 0.001 & $\begin{array}{l}\text { m28-m30: "lines" buried in the } \\
\text { substrate in five directions }\end{array}$ & \\
\hline \multicolumn{2}{|c|}{101101} & $6.7 \times 10^{-3}$ & 0.1 & & $\mathrm{~m} 39-$ Fig. $2 b$ \\
\hline
\end{tabular}

${ }^{1}$ Files were created on VT-STM Window NT system (Room 224/225 Spedding)

Path: C:IOmicronldatalsample $\backslash A \mid C u F e 5 f 2$

${ }^{2}$ Images are used in Appendix C. 
Table 5. STM database - Miscellaneous Experiments

\begin{tabular}{|c|c|c|c|c|}
\hline \multicolumn{5}{|c|}{ Lateral Calibration for VT-STM scanner } \\
\hline \multicolumn{3}{|c|}{ File Directory ${ }^{1}$} & \multicolumn{2}{|c|}{ Calibration coefficient } \\
\hline \multicolumn{3}{|c|}{ thopg } & \multicolumn{2}{|c|}{$\begin{array}{c}X \text { (lateral): } 1.23 \\
Y \text { (vertical): } 1.08\end{array}$} \\
\hline \multicolumn{5}{|c|}{ Flux Calibration for Al Evaporator - Al deposition on Al(111) } \\
\hline File Directory ${ }^{1}$ & File Name & $\begin{array}{l}\text { Dosing parameters } \\
\text { (source power, time) }\end{array}$ & $\begin{array}{c}\text { Coverage } \\
\text { (ML) }\end{array}$ & $\begin{array}{l}\text { Flux } \\
(\mathrm{ML} / \mathrm{s})\end{array}$ \\
\hline \multirow{5}{*}{ AMI00801 } & $\mathrm{m} 17$ & & 0.04 & \multirow{5}{*}{$1.7 \times 10^{-3}$} \\
\hline & $\mathrm{m} 21$ & $10 \mathrm{~V} \times 5 \mathrm{~A}, 30$ seconds & 0.04 & \\
\hline & $\mathrm{m} 25$ & \multirow{3}{*}{$10 \mathrm{~V} \times 5 \mathrm{~A}, 60 \mathrm{~seconds}$} & 0.09 & \\
\hline & $\mathrm{m} 28$ & & 0.12 & \\
\hline & $\mathrm{m} 40$ & & 0.09 & \\
\hline$\backslash A I \backslash 100901$ & $\mathrm{~m} 17$ & $8.9 \mathrm{~V} \times 4.7 \mathrm{~A}, 120$ seconds & $0.01^{(*)}$ & $8.3 \times 10^{-5(*)}$ \\
\hline \multirow[b]{2}{*}{ LAI 101001} & $\mathrm{~m} 11$ & \multirow[b]{2}{*}{$11 \mathrm{~V} \times 5.5 \mathrm{~A}, 30$ seconds } & 0.23 & \multirow[b]{2}{*}{$6.7 \times 10^{-3}$} \\
\hline & $\mathrm{m} 12$ & & 0.17 & \\
\hline \multicolumn{5}{|c|}{ Al deposition on $\mathrm{Ag}(\mathbf{1 0 0 )}$} \\
\hline \multirow[b]{2}{*}{ File Directory ${ }^{\prime}$} & \multirow[b]{2}{*}{ File Name } & \multicolumn{3}{|c|}{ Dosing parameters } \\
\hline & & Source power & & \\
\hline \multirow{3}{*}{ \Ag\101901 } & $\mathrm{m} 9-\mathrm{m} 28$ & \multirow{3}{*}{$10 \mathrm{~V} \times 5 \mathrm{~A}$} & \multicolumn{2}{|c|}{1 minute $(\tau 01 \mathrm{ML})$} \\
\hline & $\mathrm{m} 29-\mathrm{m} 44$ & & \multicolumn{2}{|c|}{2 minutes $(\sim 0.2 \mathrm{ML})$} \\
\hline & $\mathrm{m} 45-\mathrm{m} 57$ & & \multicolumn{2}{|c|}{4 minutes $(\sim 0.4 \mathrm{ML})$} \\
\hline \multirow[b]{2}{*}{ Aag\} \backslash 1 0 2 2 0 1 $&{m 1-m 12} &{\multirow[b]{2}{*}{10 \mathrm{~V} \times 5 \mathrm{~A}}1 0 \mathrm { V } \times 5 \mathrm { A }} &{\multicolumn{2}{|c|}{0.5 \text { minute }(\sim 0.05 \mathrm{ML})}0 . 5 \text { minute } ( \sim 0 . 0 5 \mathrm { ML } )} \\
{\hline} &{\mathrm{m} 14-\mathrm{m} 20} &{ } &{\multirow{2}{*}{\multicolumn{2}{|c|}{\frac{10 \text { minutes }(\sim 1.0 \mathrm{ML})}{10 \text { minutes }(\sim 0.1 \mathrm{ML})^{* * *}}}}\multicolumn { 2 } { | c | } \frac { 1 0 \text { minutes } ( \sim 1 . 0 \mathrm { ML } ) } { 1 0 \text { minutes } ( \sim 0 . 1 \mathrm { ML } ) ^ { * * * } }} \\
{\hline} &{\mathrm{m} 1-\mathrm{m} 20} &{\multirow[b]{2}{*}{9.0 \mathrm{~V} \times 4.75 \mathrm{~A}}9 . 0 \mathrm { V } \times 4 . 7 5 \mathrm { A }} &{ } &{ } \\
{\hline} &{\mathrm{m} 21-\mathrm{m} 45} &{ } &{\multicolumn{2}{|c|}{20 \text { minutes }(0.2 \mathrm{ML})^{(* *)}}2 0 \text { minutes } ( 0 . 2 \mathrm { ML } ) ^ { ( * * ) }} \\
{\hline \multirow{5}{*}{\text { IAg\102301 }}\text { IAg\102301 }} &{\mathrm{m} 1-\mathrm{m} 12} &{\multirow{5}{*}{11.0 \mathrm{~V} \times 5.5 \mathrm{~A}}1 1 . 0 \mathrm { V } \times 5 . 5 \mathrm { A }} &{\multicolumn{2}{|c|}{8 \text { seconds }(\sim 0.05 \mathrm{ML})}8 \text { seconds } ( \sim 0 . 0 5 \mathrm { ML } )} \\
{\hline} &{\mathrm{m} 13-\mathrm{m} 23} &{ } &{\multicolumn{2}{|c|}{16 \text { seconds }(\sim 0.1 \mathrm{ML})}1 6 \text { seconds } ( \sim 0 . 1 \mathrm { ML } )} \\
{\hline} &{\mathrm{m} 24-\mathrm{m} 35} &{ } &{\multicolumn{2}{|c|}{32 \text { seconds }(\sim 0.2 \mathrm{ml})}3 2 \text { seconds } ( \sim 0 . 2 \mathrm { ml } )} \\
{\hline} &{\mathrm{m} 36-\mathrm{m} 49} &{ } &{64 \text { secol }} &{0.4 \mathrm{ml})} \\
{\hline} &{\mathrm{m} 50-\mathrm{m} 70} &{ } &{2 \text { minut }} &{0.8 \mathrm{ml})} \\
$\hline}
\end{tabular}

${ }^{1}$ Files were created on VT-STM Window NT system (Room 224/225 Spedding)

Path: C:IOmicronไdatalsample

${ }^{(*)}$ The estimation of the coverage in this experiment is very crude, which may result the error in the flux calculation.

${ }^{(* *)}$ The estimation of coverage does not quite fit the obtained images, due to the inaccurate flux ${ }^{(*)}$. 


\section{ACKNOWLEDGEMENTS}

I would like to express my sincere appreciation to Professor Patricia A. Thiel for her guidance, encouragement and support through the course of my graduate study. I must also thank her for giving me the opportunity to do research in Jülich, Germany, where I obtained invaluable experience in both scientific and non-scientific respects.

My gratitude also goes to all members, former and present, in Thiel research group, who provide me the cooperative, supportive and friendly working atmosphere. I am particular thankful to Dr. Cynthia Jenks, Dr. Conrad Stoldt and Dr. Zhouxin Shen who in many ways helped me get acquainted with ultra high vacuum surface science and the field of quasicrystal at my starting point in the lab; thankful to Dr. Julian Ledieu and Dr. Dider Rouxel who I worked closely with on separate projects.

Jim Anderegg is acknowledged for his technical assistance and encouragements through our conversations. I would also like to thank Thomas Lograsso and Amy Ross for providing high quality quasicrystal samples and thank all the people who work in the quasicrystal field in Ames Laboratory.

Finally, I wish to thank my parents and sisters for the care, the faith and the moral support they have given for boosting my confidence throughout my study. With most affection, I thank my husband, Manlong who I met and married in Ames, for his continued understanding and support. 